\title{
INF-SUP STABILITY OF THE DISCRETE DUALITY FINITE VOLUME METHOD FOR THE 2D STOKES PROBLEM
}

\author{
FRANCK BOYER, STELLA KRELL, AND FLORE NABET
}

\begin{abstract}
Discrete Duality Finite Volume" schemes (DDFV for short) on general 2D meshes, in particular, non-conforming ones, are studied for the Stokes problem with Dirichlet boundary conditions. The DDFV method belongs to the class of staggered schemes since the components of the velocity and the pressure are approximated on different meshes. In this paper, we investigate from a numerical and theoretical point of view, whether or not the stability condition holds in this framework for various kinds of mesh families. We obtain that different behaviors may occur depending on the geometry of the meshes.

For instance, for conforming acute triangle meshes, we prove the unconditional Inf-Sup stability of the scheme, whereas for some conforming or nonconforming Cartesian meshes we prove that Inf-Sup stability holds up to a single unstable pressure mode. In any case, the DDFV method appears to be very robust.
\end{abstract}

\section{INTRODUCTION}

1.1. The Stokes problem. In this paper, we are concerned with a finite volume approximation of the following 2D incompressible Stokes problem: Find a velocity field $\mathbf{u}: \Omega \rightarrow \mathbb{R}^{2}$ and a pressure field $p: \Omega \rightarrow \mathbb{R}$,

$$
\left\{\begin{array}{rlrl}
-\Delta \mathbf{u}+\nabla p & =\mathbf{f}, & \text { in } \Omega, \\
\operatorname{div} \mathbf{u} & =0, & \text { in } \Omega, \\
\mathbf{u} & =0, & \text { on } \partial \Omega, & m(p) \stackrel{\text { def }}{=} \frac{1}{m_{\Omega}} \int_{\Omega} p=0 .
\end{array}\right.
$$

We assume that $\Omega$ is a bounded connected polygonal domain in $\mathbb{R}^{2}, m_{\Omega}$ being its Lebesgue measure, and $\mathbf{f}$ is a function in $\left(L^{2}(\Omega)\right)^{2}$.

We recall that the well-posedness of this problem is related to the validity of the so-called Inf-Sup (or LBB) inequality

$$
\inf _{p \in L_{0}^{2}(\Omega)}\left(\sup _{\mathbf{v} \in\left(H_{0}^{1}(\Omega)\right)^{2}} \frac{b(\mathbf{v}, p)}{\|\mathbf{v}\|_{H^{1}}\|p\|_{L^{2}}}\right)>0,
$$

where $b(\mathbf{v}, p)=\int_{\Omega} p(\operatorname{div} \mathbf{v})$ and $L_{0}^{2}(\Omega)=\left\{p \in L^{2}(\Omega): m(p)=0\right\}$. This inequality is itself known to be equivalent to the existence of a continuous right-inverse of the divergence operator stated in the following result (see [8,22]).

Received by the editor February 27, 2013 and, in revised form, December 20, 2013 and March 12,2014 .

2010 Mathematics Subject Classification. Primary 65N08, 65N12, 76D07, 76M12.

Key words and phrases. Finite-volume methods, Stokes problem, DDFV methods, Inf-Sup stability. 
Proposition 1.1. There exists a linear continuous operator $\Pi: L_{0}^{2}(\Omega) \rightarrow\left(H_{0}^{1}(\Omega)\right)^{2}$ such that

$$
\operatorname{div}(\Pi(p))=p, \quad \forall p \in L_{0}^{2}(\Omega) .
$$

1.2. Finite volume methods for the Stokes problem. Finite volume methods have been extensively studied for a long time in several engineering fields. Indeed, they are well suited for the numerical approximation of conservation laws appearing for instance in fluid mechanics, petroleum engineering and many other fields. The theoretical analysis of finite volume schemes (convergence analysis, error estimates,...) began at the end of the 1980s and had a rapid expansion during the 1990s; see for instance the book by Eymard, Gallouët, Herbin [19] and all the references therein.

Finite volume approximation of Stokes problems is a current research topic and can be split into two families of methods: collocated and staggered. Let us cite for instance the Mimetic Finite Difference method [4 6], the Discrete Duality Finite Volume schemes (DDFV for short) [14,26], the Mixed Finite Volume schemes [17, the Scheme Using Stabilization and Hybrid Interfaces [20]. The most celebrated staggered scheme is the MAC scheme [24,29] on Cartesian grids.

In this paper, we focus on a DDFV approximation of Stokes equations. It is a staggered method since the approximate velocity field and pressure field are associated with different control volumes. Actually, for a Cartesian grid, the scheme we propose here is equivalent (except on the boundary) to two uncoupled MAC schemes written on two different staggered meshes. Therefore, the DDFV method for the Stokes problem can be considered as a possible extension of MAC to general meshes.

The 2D DDFV scheme requires velocity unknowns on both vertices and "centers" of control volumes. These two sets of unknowns allow us to reconstitute a twodimensional discrete gradient (defined on new geometric elements called diamond cells) and discrete divergence operators that are in duality in a discrete sense giving its name to the method. This approach was first introduced in [14] but some important points of the analysis were left open, such as the question of uniform Inf-Sup stability of the method which is the main topic of our work.

Note that, to overcome these difficulties in the analysis, the author of 14 proposed to formulate the Stokes problem in the vorticity-velocity-pressure form and then to approximate the velocity on the diamond cells and the pressure on both vertices and centers of primal control volumes. This dual approach does not seem to be adapted to Dirichlet boundary conditions or more general problems such as the multifluid Stokes problem for which the viscosity is no more constant on $\Omega$. That's the reason why we believe that the study of the DDFV method in the natural velocity/pressure formulation is still an important topic.

In order to cope with the lack, at that time, of a suitable discrete Inf-Sup inequality for the natural DDFV formulation, it was also proposed in [26] to add a stabilization term in the mass conservation equation. With this usual stabilization technique, a complete analysis of the scheme was given. However, it was numerically observed in this reference that very accurate approximations can be computed without stabilization (or at least with very small stabilization parameters).

This is our main motivation in the present paper to go further in the analysis of the Inf-Sup stability of the original (non-stabilized) DDFV scheme. This scheme is easy to implement on general grids (with a single loop over diamond cells), has 
a reasonably small stencil, is parameter-free and possesses the standard algebraic saddle-point structure well-suited to many iterative solvers and preconditioners. Moreover, using the Inf-Sup stability results proved in this paper, the error analysis presented in [26] directly applies to the non-stabilized scheme. We only consider here the $2 \mathrm{D}$ case but it is worth noticing that DDFV schemes have been successfully extended to the 3D case in [1,11, 12,25, for linear anisotropic scalar diffusion equations and in 27. for the Stokes problem.

The Inf-Sup stability issue has been extensively studied in the framework of conforming Galerkin/finite element approximations and the main results in this field can be found in [9, 18,22 for instance; see also the review paper [7. In many cases, the Inf-Sup stability can be analysed by proving the existence of the socalled Fortin operator as introduced initially in [21. This strategy was for instance successfully used for the Crouzeix-Raviart element [13] or the $\mathbb{P}^{1}+$ bubble $/ \mathbb{P}^{1}$ (mini) element $\left[3\right.$. For the generalised $\mathbb{P}^{2} / \mathbb{P}^{1}$ Hood-Taylor element or its linear counterpart $\mathbb{P}^{1}$ iso $\mathbb{P}^{2} / \mathbb{P}^{1}$, an adaptation of Fortin's method is necessary [31]. The proofs given in the present paper can actually be seen as adaptations to the DDFV framework of Verfürth's ideas, even though our approximate pressure field is not continuous.

As far as discontinuous Galerkin methods are concerned (see [15] for a more detailed review of this class of methods), we can cite for instance [10] where the LDG method in variables velocity-velocity gradient-pressure is analysed in detail, in particular its Inf-Sup stability properties. Since this method is locally conservative, it can be understood in some sense as a higher order finite volume approximation. However, this method requires a pressure stabilization term in the mass conservation equation. Another DG method in the velocity/pressure formulation without pressure stabilization (at least on matching simplicial grids) is analyzed in [23. None of these methods is able to cope with general grids without pressure stabilization contrary to the DDFV method presented here. This is an important feature of staggered methods.

1.3. Outline. This article is organized as follows. In Section 2, we first recall the DDFV framework, introduce the DDFV scheme and define the associated discrete Inf-Sup condition. In Section 3, we first study three examples of different mesh families for which we are able to prove the unconditional Inf-Sup stability of the scheme. We also provide numerical illustrations for these properties.

Conversely, in Section 4, we prove that for some other mesh families (of Cartesian type), the Inf-Sup stability does not hold. However, we can provide a precise description of the instability by establishing that there exists only one single unstable mode in such a way that the Inf-Sup stability holds if we impose to the pressure fields to be orthogonal to the unstable mode. This seems to explain why, in that case, the Inf-Sup instability of the scheme is sufficiently weak so that the convergence properties of the method are preserved even without a strong stabilization term as observed in [26].

The general idea underlying our analysis is to build a kind of approximate Fortin operator, since building a real Fortin operator seems to be complicated, in particular, for non-conforming meshes (see Propositions 3.1 and 4.5).

Finally, in Section 5, we propose some numerical experiments to check whether or not the Inf-Sup stability holds for different mesh families for which we are not yet able to provide theoretical results. The conclusion of the study is that the DDFV seems to be very robust as far as the Inf-Sup stability property is concerned. 


\section{DDFV FRAMEWORK}

2.1. The DDFV meshes and notation. For any two vectors $\mathbf{a}, \mathbf{b}$ in $\mathbb{R}^{2}$, we denote by $\mathbf{a} \cdot \mathbf{b}={ }^{t} \mathbf{a b} \in \mathbb{R}$ their euclidean scalar product, by $\mathbf{a} \otimes \mathbf{b}=\mathbf{a}^{t} \mathbf{b} \in \mathcal{M}_{2}(\mathbb{R})$ their tensor product and by $\mathbf{a} \wedge \mathbf{b}=\operatorname{det}(\mathbf{a}, \mathbf{b}) \in \mathbb{R}$ their wedge product. Moreover, $\mathbf{e}_{\boldsymbol{x}}={ }^{t}\left(\begin{array}{ll}1 & 0)\end{array}\right)$ and $\mathbf{e}_{\boldsymbol{y}}={ }^{t}\left(\begin{array}{ll}0 & 1\end{array}\right)$ denote the canonical basis of $\mathbb{R}^{2}$.

For any two matrices $\xi, \tilde{\xi} \in \mathcal{M}_{2}(\mathbb{R})$, we denote by $(\xi: \tilde{\xi})=\operatorname{Tr}\left({ }^{t} \xi \tilde{\xi}\right) \in \mathbb{R}$ their contracted product and by $|\xi|=(\xi: \xi)^{1 / 2}$ the associated norm.

The meshes. We recall here the main notations and definitions taken from [2]. A DDFV mesh $\mathcal{T}$ is constituted by a primal mesh $\mathfrak{M} \cup \partial \mathfrak{M}$ and a dual mesh $\mathfrak{M}^{*} \cup \partial \mathfrak{M}^{*}$. An example for square locally refined primal mesh is in Figure 1.

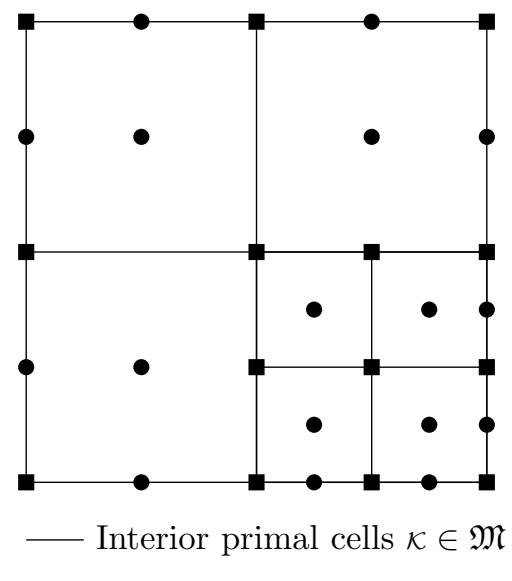

- Centers $x_{\mathcal{K}}$

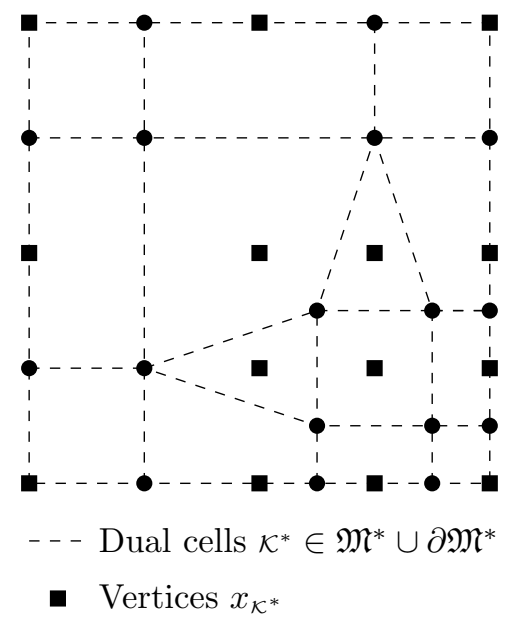

- Vertices $x_{\mathcal{K}^{*}}$

Figure 1. (Left) The primal mesh $\mathfrak{M} \cup \partial \mathfrak{M}$; (Right) The dual mesh $\mathfrak{M}^{*} \cup \partial \mathfrak{M}^{*}$.

The (interior) primal mesh $\mathfrak{M}$ is a set of disjoint open polygonal control volumes $\mathcal{K} \subset \Omega$ such that $\bigcup \overline{\mathcal{K}}=\bar{\Omega}$. We denote by $\partial \mathfrak{M}$ the set of edges of the control volumes in $\mathfrak{M}$ included in $\partial \Omega$, which we consider as degenerate control volumes.

- To each control volume $\mathcal{K} \in \mathfrak{M}$, we associate a point $x_{\mathcal{K}}$. Even though many choices are possible, in this paper, we always assume $x_{\mathcal{K}}$ to be the mass center of $\mathcal{K}$.

- To each degenerate control volume $\mathcal{K} \in \partial \mathfrak{M}$, we associate the point $x_{\mathcal{K}}$ equal to the midpoint of the control volume $\mathcal{K}$.

This family of points is denoted by $X=\left\{x_{\mathcal{K}}, \mathcal{K} \in \mathfrak{M} \cup \partial \mathfrak{M}\right\}$.

Let $X^{*}$ denote the set of the vertices of the primal control volumes in $\mathfrak{M}$ that we split into $X^{*}=X_{\text {int }}^{*} \cup X_{\text {ext }}^{*}$ where $X_{\text {int }}^{*} \cap \partial \Omega=\emptyset$ and $X_{\text {ext }}^{*} \subset \partial \Omega$. With any point $x_{\mathcal{K}^{*}} \in X_{\text {int }}^{*}$ (resp. $x_{\mathcal{K}^{*}} \in X_{\text {ext }}^{*}$ ), we associate the polygon $\mathcal{K}^{*} \in \mathfrak{M}^{*}$ (resp. $\left.\mathcal{K}^{*} \in \partial \mathfrak{M}^{*}\right)$ whose vertices are $\left\{x_{\mathcal{K}} \in X\right.$, such that $\left.x_{\mathcal{K}^{*}} \in \overline{\mathcal{K}}, \mathcal{K} \in \mathfrak{M}\right\}$ (resp. $\left\{x_{\mathcal{K}^{*}}\right\} \cup\left\{x_{\mathcal{K}} \in X\right.$, such that $\left.\left.x_{\mathcal{K}^{*}} \in \overline{\mathcal{K}}, \mathcal{K} \in(\mathfrak{M} \cup \partial \mathfrak{M})\right\}\right)$ sorted with respect to the clockwise order of the corresponding control volumes. This defines the set $\mathfrak{M}^{*} \cup \partial \mathfrak{M}^{*}$ of dual control volumes. 
For all control volumes $\mathcal{K}$ and $\mathcal{L}$, we assume that $\partial \mathcal{K} \cap \partial \mathcal{L}$ is either empty or a common vertex or an edge of the primal mesh denoted by $\sigma=\mathcal{K} \mid \mathcal{L}$. We note by $\mathcal{E}$ the set of such edges. We also denote $\sigma^{*}=\mathcal{K}^{*} \mid \mathcal{L}^{*}$ and $\mathcal{E}^{*}$ for the corresponding dual definitions.

Given the primal and dual control volumes, we define the diamond cells $\mathcal{D}_{\sigma, \sigma^{*}}$ as being the quadrangles whose diagonals are a primal edge $\sigma=\mathcal{K} \mid \mathcal{L}=\left(x_{\mathcal{K}^{*}}, x_{\mathcal{L}^{*}}\right)$ and a corresponding dual edge $\sigma^{*}=\mathcal{K}^{*} \mid \mathcal{L}^{*}=\left(x_{\mathcal{K}}, x_{\mathcal{L}}\right)$, (see Figure 2). Note that the diamond cells are not necessarily convex. If $\sigma \in \mathcal{E} \cap \partial \bar{\Omega}$, the quadrangle $\mathcal{D}_{\sigma, \sigma^{*}}$ degenerate into a triangle. The set of the diamond cells is denoted by $\mathfrak{D}$ and we have $\bar{\Omega}=\underset{\mathcal{D} \in \mathfrak{D}}{\cup} \overline{\mathcal{D}}$.

Notations. For any primal control volume $\mathcal{K} \in \mathfrak{M} \cup \partial \mathfrak{M}$, we note:

- $m_{\mathcal{K}}$ its Lebesgue measure,

- $\mathcal{E}_{\mathcal{K}}$ the set of its edges (if $\mathcal{K} \in \mathfrak{M}$ ), or the one-element set $\{\mathcal{K}\}$ if $\mathcal{K} \in \partial \mathfrak{M}$.

- $\mathfrak{D}_{\mathcal{K}}=\left\{\mathcal{D}_{\sigma, \sigma^{*}} \in \mathfrak{D}, \sigma \in \mathcal{E}_{\mathcal{K}}\right\}$,

- $h_{\mathcal{K}}$ its diameter.

We will also use corresponding dual notations: $m_{\mathcal{K}^{*}}, \mathcal{E}_{\mathcal{K}^{*}}, \mathfrak{D}_{\mathcal{K}^{*}}$ and $h_{\mathcal{K}^{*}}$.

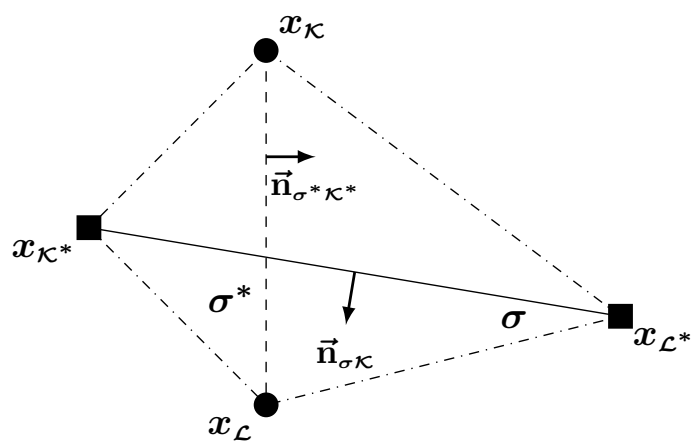

Figure 2. Notations in a diamond cell $\mathcal{D}$

For a diamond cell $\mathcal{D}=\mathcal{D}_{\sigma, \sigma^{*}}$ whose vertices are $\left(x_{\mathcal{K}}, x_{\mathcal{K}^{*}}, x_{\mathcal{L}}, x_{\mathcal{L}^{*}}\right)$ (see Figure 2), we note that:

- $m_{\sigma}$ the length of the primal edge $\sigma$,

- $m_{\sigma^{*}}$ the length of the dual edge $\sigma^{*}$,

- $\overrightarrow{\mathbf{n}}_{\sigma \mathcal{K}}$ the unit vector normal to $\sigma$ oriented from $x_{\mathcal{K}}$ to $x_{\mathcal{L}}$,

- $\overrightarrow{\mathbf{n}}_{\sigma^{*} \mathcal{K}^{*}}$ the unit vector normal to $\sigma^{*}$ oriented from $x_{\mathcal{K}^{*}}$ to $x_{\mathcal{L}^{*}}$,

- $h_{\mathcal{D}}$ its diameter,

- $m_{\mathcal{D}}$ its measure.

We define the set of boundary diamond cells $\mathfrak{D}_{\text {ext }}$ as the set of diamond cells which possess one side included in $\partial \Omega$; the set of interior diamond cells is thus $\mathfrak{D}_{\text {int }}=\mathfrak{D} \backslash \mathfrak{D}_{\text {ext }}$.

Mesh regularity measurement. Let $\operatorname{size}(\mathcal{T})$ be the maximum of the diameters of the diamond cells in $\mathfrak{D}$. We introduce a positive number $\operatorname{reg}(\mathcal{T})$ that measures the regularity of a given mesh and is useful to perform the convergence analysis of 
finite volume schemes

$$
\begin{array}{r}
\operatorname{reg}(\mathcal{T})=\max \left(\mathcal{N}, \mathcal{N}^{*}, \max _{\mathcal{D} \in \mathfrak{D}} \frac{m_{\sigma} m_{\sigma^{*}}}{m_{\mathcal{D}}}, \max _{\substack{\mathcal{K} \in \mathfrak{M} \\
\mathcal{D} \in \mathcal{O} \mathcal{K}}} \frac{h_{\mathcal{K}}}{h_{\mathcal{D}}}, \max _{\substack{\mathcal{K}^{*} \in \mathfrak{M} * \cup \partial \mathfrak{M}^{*} \\
\mathcal{D} \in \mathfrak{D} \mathcal{K}^{*}}} \frac{h_{\mathcal{K}^{*}}}{h_{\mathcal{D}}},\right. \\
\left.\max _{\mathcal{D} \in \mathfrak{D}} \frac{h_{\mathcal{D}}}{\sqrt{m_{\mathcal{D}}}}, \max _{\mathcal{K}^{*} \in \mathfrak{M}^{*} \cup \partial \mathfrak{M}^{*}} \frac{h_{\mathcal{K}^{*}}}{\sqrt{m_{\mathcal{K}^{*}}}}, \max _{\mathcal{K} \in \mathfrak{M}} \frac{h_{\mathcal{K}}}{\sqrt{m_{\mathcal{K}}}}\right),
\end{array}
$$

where $\mathcal{N}$ and $\mathcal{N}^{*}$ are the maximum of edges of each primal cell and the maximum of edges incident to any vertex. The number $\operatorname{reg}(\mathcal{T})$ should be uniformly bounded when $\operatorname{size}(\mathcal{T}) \rightarrow 0$ for the convergence results to hold.

2.2. Discrete unknowns and discrete mean-value projection. The DDFV method for the Stokes problem requires staggered unknowns. It associates to any primal cell $\mathcal{K} \in \mathfrak{M} \cup \partial \mathfrak{M}$ an unknown value $\mathbf{u}_{\mathcal{K}} \in \mathbb{R}^{2}$ for the velocity, to any dual cell $\mathcal{K}^{*} \in \mathfrak{M}^{*} \cup \partial \mathfrak{M}^{*}$ an unknown value $\mathbf{u}_{\mathcal{K}^{*}} \in \mathbb{R}^{2}$ for the velocity and to any diamond cell $\mathcal{D} \in \mathfrak{D}$ an unknown value $p^{\mathcal{D}} \in \mathbb{R}$ for the pressure. These unknowns are collected in the families

$$
\mathbf{u}^{\mathcal{T}}=\left(\begin{array}{c}
\mathbf{u}^{\mathfrak{M}}=\left(\mathbf{u}_{\mathcal{K}}\right)_{\mathcal{K} \in \mathfrak{M}} \\
\mathbf{u}^{\partial \mathfrak{M}}=\left(\mathbf{u}_{\mathcal{K}}\right)_{\mathcal{K} \in \partial \mathfrak{M}} \\
\mathbf{u}^{\mathfrak{M}^{*}}=\left(\mathbf{u}_{\mathcal{K}^{*}}\right)_{\mathcal{K}^{*} \in \mathfrak{M}^{*}} \\
\mathbf{u}^{\partial \mathfrak{M}^{*}}=\left(\mathbf{u}_{\mathcal{K}^{*}}\right)_{\mathcal{K}^{*} \in \partial \mathfrak{M} *}
\end{array}\right) \in\left(\mathbb{R}^{2}\right)^{\mathcal{T}} \text { and } p^{\mathfrak{D}}=\left(\left(p^{\mathcal{D}}\right)_{\mathcal{D} \in \mathfrak{D}}\right) \in \mathbb{R}^{\mathfrak{D}}
$$

We specify a subset of $\left(\mathbb{R}^{2}\right)^{\mathcal{T}}$ needed to take into account the Dirichlet boundary conditions

$$
\mathbb{E}_{0}=\left\{\mathbf{u}^{\mathcal{T}} \in\left(\mathbb{R}^{2}\right)^{\mathcal{T}} \text { such that } \mathbf{u}^{\partial \mathfrak{M}}=0 \text { and } \mathbf{u}^{\partial \mathfrak{M}^{*}}=0\right\}
$$

We define now the interior mean-value projection for any vector field $\mathbf{v} \in\left(H_{0}^{1}(\Omega)\right)^{2}$ $(2.2)$

$$
\mathbb{P}_{\boldsymbol{m}}^{\mathfrak{M}} \mathbf{v}=\left(\left(\frac{1}{m_{\mathcal{K}}} \int_{\mathcal{K}} \mathbf{v}(x) \mathrm{d} x\right)_{\mathcal{K} \in \mathfrak{M}}\right), \mathbb{P}_{\boldsymbol{m}}^{\mathfrak{M}^{*}} \mathbf{v}=\left(\left(\frac{1}{m_{\mathcal{K}^{*}}} \int_{\mathcal{K}^{*}} \mathbf{v}(x) \mathrm{d} x\right)_{\mathcal{K}^{*} \in \mathfrak{M}^{*}}\right) .
$$

We finally gather these projections in the following notation:

$$
\mathbb{P}_{\boldsymbol{m}}^{\boldsymbol{T}} \mathbf{v}=\left(\begin{array}{c}
\mathbb{P}_{\boldsymbol{m}}^{\mathfrak{M}} \mathbf{v} \\
0 \\
\mathbb{P}_{\boldsymbol{m}}^{\mathfrak{M}^{*}} \mathbf{v} \\
0
\end{array}\right) \in \mathbb{E}_{0}, \quad \forall \mathbf{v} \in\left(H_{0}^{1}(\Omega)\right)^{2}
$$

2.3. Discrete operators. In this subsection, we define the discrete operators which are needed in order to write and analyse the DDFV scheme. We begin with the discrete gradient.

Definition 2.1. We define the discrete gradient operator $\nabla^{\mathfrak{D}}$ mapping vector fields of $\left(\mathbb{R}^{2}\right)^{\mathcal{T}}$ into matrix fields of $\left(\mathcal{M}_{2}(\mathbb{R})\right)^{\mathfrak{D}}$, as

$$
\nabla^{\mathcal{D}} \mathbf{u}^{\mathcal{T}}=\frac{1}{2 m_{\mathcal{D}}}\left[m_{\sigma}\left(\mathbf{u}_{\mathcal{L}}-\mathbf{u}_{\mathcal{K}}\right) \otimes \overrightarrow{\mathbf{n}}_{\sigma \mathcal{K}}+m_{\sigma^{*}}\left(\mathbf{u}_{\mathcal{L}^{*}}-\mathbf{u}_{\mathcal{K}^{*}}\right) \otimes \overrightarrow{\mathbf{n}}_{\sigma^{*} \mathcal{K}^{*}}\right], \forall \mathcal{D} \in \mathfrak{D},
$$

for any $\mathbf{u}^{\mathcal{T}} \in\left(\mathbb{R}^{2}\right)^{\mathcal{T}}$. 
Definition 2.2. We define the discrete divergence operator $\operatorname{div}^{\mathfrak{D}}$ mapping vector fields of $\left(\mathbb{R}^{2}\right)^{\mathcal{T}}$ into scalar fields in $\mathbb{R}^{\mathfrak{D}}$, as

$$
\operatorname{div}^{\mathcal{D}} \mathbf{u}^{\mathcal{T}}=\operatorname{Tr}\left(\nabla^{\mathcal{D}} \mathbf{u}^{\mathcal{T}}\right)=\frac{1}{2 m_{\mathcal{D}}}\left[m_{\sigma}\left(\mathbf{u}_{\mathcal{L}}-\mathbf{u}_{\mathcal{K}}\right) \cdot \overrightarrow{\mathbf{n}}_{\sigma \mathcal{K}}+m_{\sigma^{*}}\left(\mathbf{u}_{\mathcal{L}^{*}}-\mathbf{u}_{\mathcal{K}^{*}}\right) \cdot \overrightarrow{\mathbf{n}}_{\sigma^{*} \mathcal{K}^{*}}\right]
$$

for any $\mathcal{D} \in \mathfrak{D}$ and any $\mathbf{u}^{\mathcal{T}} \in\left(\mathbb{R}^{2}\right)^{\mathcal{T}}$.

Definition 2.3. We define the discrete divergence operator $\operatorname{div}^{\mathcal{T}}$ mapping matrix fields in $\left(\mathcal{M}_{2}(\mathbb{R})\right)^{\mathfrak{D}}$ into vector fields in $\mathbb{E}_{0}$, as

$$
\left\{\begin{aligned}
\operatorname{div}^{\mathcal{K}} \xi^{\mathcal{D}} & =\frac{1}{m_{\mathcal{K}}} \sum_{\sigma \in \partial \mathcal{K}} m_{\sigma} \xi^{\mathcal{D}} \overrightarrow{\mathbf{n}}_{\sigma \mathcal{K}}, \forall \mathcal{K} \in \mathfrak{M}, \\
\operatorname{div}^{\mathcal{K}^{*}} \xi^{\mathcal{D}} & =\frac{1}{m_{\mathcal{K}^{*}}} \sum_{\sigma^{*} \in \partial \mathcal{K}^{*}} m_{\sigma^{*}} \xi^{\mathcal{D}} \overrightarrow{\mathbf{n}}_{\sigma^{*} \mathcal{K}^{*}}, \forall \mathcal{K}^{*} \in \mathfrak{M}^{*},
\end{aligned}\right.
$$

for any $\xi^{\mathfrak{D}} \in\left(\mathcal{M}_{2}(\mathbb{R})\right)^{\mathfrak{D}}$.

In order to write the DDFV scheme in a compact form, we will denote the discrete divergence on the primal mesh and the one on the interior dual mesh as:

$$
\operatorname{div}^{\mathfrak{M}} \xi^{\mathfrak{D}}=\left(\operatorname{div}^{\mathcal{K}} \xi^{\mathfrak{D}}\right)_{\mathcal{K} \in \mathfrak{M}}, \quad \operatorname{div}^{\mathfrak{M}^{*}} \xi^{\mathfrak{D}}=\left(\operatorname{div}^{\mathcal{K}^{*}} \xi^{\mathfrak{D}}\right)_{\mathcal{K}^{*} \in \mathfrak{M}^{*}} .
$$

Definition 2.4. We define the discrete gradient operator $\nabla^{\mathcal{T}}$ mapping scalar fields $\mathbb{R}^{\mathfrak{D}}$ into vector fields in $\mathbb{E}_{0}$ as follows:

$$
\nabla^{\mathcal{T}} p^{\mathfrak{D}}=\operatorname{div}^{\mathcal{T}}\left(p^{\mathfrak{D}} \mathrm{Id}\right), \forall p^{\mathfrak{D}} \in \mathbb{R}^{\mathfrak{D}} .
$$

Remark 2.5. We emphasize that, by definition, $\operatorname{div}^{\mathcal{K}^{*}} \xi^{\mathfrak{D}}$ and $\nabla^{\mathcal{K}^{*}} p^{\mathfrak{D}}$ are set to 0 for boundary dual cells $\mathcal{K}^{*} \in \partial \mathfrak{M}^{*}$.

In short, we have introduced four operators:

$$
\begin{aligned}
\nabla^{\mathfrak{D}} & :\left(\mathbb{R}^{2}\right)^{\mathcal{T}} \rightarrow\left(\mathcal{M}_{2}(\mathbb{R})\right)^{\mathfrak{D}}, \\
\operatorname{div}^{\mathfrak{D}} & :\left(\mathbb{R}^{2}\right)^{\mathcal{T}} \rightarrow \mathbb{R}^{\mathfrak{D}}, \\
\operatorname{div}^{\mathcal{T}}: & \left(\mathcal{M}_{2}(\mathbb{R})\right)^{\mathfrak{D}} \rightarrow \mathbb{E}_{0}, \\
\nabla^{\mathcal{T}} & : \mathbb{R}^{\mathfrak{D}} \rightarrow \mathbb{E}_{0} .
\end{aligned}
$$

2.4. Discrete inner product and norms. First of all, we define the three following inner products:

$$
\begin{aligned}
& \llbracket \mathbf{u}^{\mathcal{T}}, \mathbf{v}^{\mathcal{T}} \rrbracket_{\mathcal{T}}=\frac{1}{2}\left(\sum_{\mathcal{K} \in \mathfrak{M}} m_{\mathcal{K}} \mathbf{u}_{\mathcal{K}} \cdot \mathbf{v}_{\mathcal{K}}+\sum_{\mathcal{K}^{*} \in \mathfrak{M}^{*}} m_{\mathcal{K}^{*}} \mathbf{u}_{\mathcal{K}^{*}} \cdot \mathbf{v}_{\mathcal{K}^{*}}\right), \quad \forall \mathbf{u}^{\mathcal{T}}, \mathbf{v}^{\mathcal{T}} \in \mathbb{E}_{0}, \\
& \left(p^{\mathfrak{D}}, q^{\mathfrak{D}}\right)_{\mathfrak{D}}=\sum_{\mathcal{D} \in \mathfrak{D}} m_{\mathcal{D}} p^{\mathcal{D}} q^{\mathcal{D}}, \quad \forall p^{\mathfrak{D}}, q^{\mathfrak{D}} \in \mathbb{R}^{\mathfrak{D}}, \\
& \left(\xi^{\mathfrak{D}}: \phi^{\mathfrak{D}}\right)_{\mathfrak{D}}=\sum_{\mathcal{D} \in \mathfrak{D}} m_{\mathcal{D}}\left(\xi^{\mathcal{D}}: \phi^{\mathcal{D}}\right), \quad \forall \xi^{\mathfrak{D}}, \phi^{\mathfrak{D}} \in\left(\mathcal{M}_{2}(\mathbb{R})\right)^{\mathfrak{D}} .
\end{aligned}
$$

Then, we define the corresponding norms as follows:

$$
\begin{array}{rlrl}
\left\|\mathbf{u}^{\mathcal{T}}\right\|_{\mathcal{T}, 2} & =\llbracket \mathbf{u}^{\mathcal{T}}, \mathbf{u}^{\mathcal{T}} \rrbracket_{\mathcal{T}}^{\frac{1}{2}}, & & \forall \mathbf{u}^{\mathcal{T}} \in \mathbb{E}_{0}, \\
\left\|p^{\mathfrak{D}}\right\|_{\mathfrak{D}, 2}=\left(p^{\mathfrak{D}}, p^{\mathfrak{D}}\right)_{\mathfrak{D}}^{\frac{1}{2}}, & \forall p^{\mathfrak{D}} \in \mathbb{R}^{\mathfrak{D}}, \\
\left\|\xi^{\mathfrak{D}}\right\|_{\mathfrak{D}, 2}=\left(\xi^{\mathfrak{D}}: \xi^{\mathfrak{D}}\right)_{\mathfrak{D}}^{\frac{1}{2}}, & \forall \xi^{\mathfrak{D}} \in\left(\mathcal{M}_{2}(\mathbb{R})\right)^{\mathfrak{D}} .
\end{array}
$$

The following discrete Stokes formula holds, giving its name to the Discrete Duality Method (see for instance, 2, 16]). 
Theorem 2.6 (Discrete Stokes formula). For all $\xi^{\mathfrak{D}} \in\left(\mathcal{M}_{2}(\mathbb{R})\right)^{\mathfrak{D}}$, $\mathbf{u}^{\mathcal{T}} \in \mathbb{E}_{0}$, we have

$$
\llbracket \operatorname{div}^{\mathcal{T}} \xi^{\mathfrak{D}}, \mathbf{u}^{\mathcal{T}} \rrbracket_{\mathcal{T}}=-\left(\xi^{\mathfrak{D}}: \nabla^{\mathfrak{D}} \mathbf{u}^{\mathcal{T}}\right)_{\mathfrak{D}}
$$

We finally recall (see for instance, [2]) that $\mathbf{v}^{\mathcal{T}} \in \mathbb{E}_{0} \mapsto\left\|\nabla^{\mathfrak{D}} \mathbf{v}^{\mathcal{T}}\right\|_{\mathfrak{D}, 2}$ is a norm in $\mathbb{E}_{0}$ (actually, a suitable Poincaré inequality holds) and that for some $C_{1}>0$ depending only on $\operatorname{reg}(\mathcal{T})$, we have the stability estimate

$$
\left\|\nabla^{\mathfrak{D}} \mathbb{P}_{\boldsymbol{m}}^{\mathcal{T}} \mathbf{v}\right\|_{\mathfrak{D}, 2} \leq C_{1}\|\mathbf{v}\|_{H^{1}}, \forall \mathbf{v} \in\left(H_{0}^{1}(\Omega)\right)^{2} .
$$

2.5. Stokes-DDFV scheme. The DDFV scheme for Problem (1.1) reads as follows: Find $\mathbf{u}^{\mathcal{T}} \in \mathbb{E}_{0}$ and $p^{\mathfrak{D}} \in \mathbb{R}^{\mathfrak{D}}$ such that

$$
\left\{\begin{aligned}
\operatorname{div}^{\mathfrak{M}}\left(-\nabla^{\mathfrak{D}} \mathbf{u}^{\mathcal{T}}+p^{\mathfrak{D}} \mathrm{Id}\right) & =\mathbf{f}^{\mathfrak{M}}, \\
\operatorname{div}^{\mathfrak{M}}{ }^{*}\left(-\nabla^{\mathfrak{D}} \mathbf{u}^{\mathcal{T}}+p^{\mathfrak{D}} \mathrm{Id}\right) & =\mathbf{f}^{\mathfrak{M}^{*}}, \\
\operatorname{div}^{\mathfrak{D}} \mathbf{u}^{\mathcal{T}} & =0, \\
m\left(p^{\mathfrak{D}}\right)=\sum_{\mathcal{D} \in \mathfrak{D}} m_{\mathcal{D}} p^{\mathcal{D}} & =0,
\end{aligned}\right.
$$

with $\mathbf{f}^{\mathfrak{M}}=\mathbb{P}_{\boldsymbol{m}^{\mathfrak{M}}}^{\mathbf{f}}$ and $\mathbf{f}^{\mathfrak{M}^{*}}=\mathbb{P}_{\boldsymbol{m}}^{\mathfrak{M}^{*}} \mathbf{f}$, where the projection is defined by (2.2).

This scheme is formally obtained by integrating the momentum equation in problem (1.1) on the primal mesh $\mathfrak{M}$ and on the interior dual mesh $\mathfrak{M}^{*}$ and the mass conservation equation on the diamond mesh $\mathfrak{D}$. The momentum and mass fluxes are then approximated by using the DDFV gradients as defined in the previous section. The homogeneous Dirichlet boundary conditions are specified on $\partial \mathfrak{M}$ and on $\partial \mathfrak{M}^{*}$ through the definition of the space $\mathbb{E}_{0}$.

We also want to emphasize that the practical implementation of the scheme is easy since, for any kind of mesh, each numerical flux that needs to be evaluated in the momentum equation depends, at most, on four velocity unknowns. Moreover, the matrix of the system (see Section 2.6.2) can be assembled diamond cell by diamond cell.

In 14] the author shows that for an acute triangle mesh or a non-conforming rectangle mesh, then problem (2.5) has a unique solution. However, no stability estimate was derived even in that cases, that is the reason why we are interested in studying the discrete Inf-Sup condition for this scheme.

\subsection{Discrete Inf-Sup constant.}

2.6.1. Definition. Given a DDFV mesh $\mathcal{T}$, we define the discrete Inf-Sup constant $\beta_{\mathcal{T}}$ associated with the scheme (2.5) as

$$
\beta_{\mathcal{T}}=\inf _{p^{\mathfrak{D}} \in \mathbb{R}^{\mathfrak{D}}}\left(\sup _{\mathbf{v}^{\mathcal{T}} \in \mathbb{E}_{0}} \frac{b_{\mathcal{T}}\left(\mathbf{v}^{\mathcal{T}}, p^{\mathfrak{D}}\right)}{\left\|\nabla^{\mathfrak{D}} \mathbf{v}^{\mathcal{T}}\right\|_{\mathfrak{D}, 2}\left\|p^{\mathfrak{D}}-m\left(p^{\mathfrak{D}}\right)\right\|_{\mathfrak{D}, 2}}\right),
$$

where

$$
b_{\mathcal{T}}\left(\mathbf{v}^{\mathcal{T}}, p^{\mathfrak{D}}\right)=\left(\operatorname{div}^{\mathfrak{D}} \mathbf{v}^{\mathcal{T}}, p^{\mathfrak{D}}\right)_{\mathfrak{D}}=-\llbracket \mathbf{v}^{\mathcal{T}}, \nabla^{\mathcal{T}} p^{\mathfrak{D}} \rrbracket_{\mathcal{T}}, \quad \forall \mathbf{v}^{\mathcal{T}} \in \mathbb{E}_{0}, \forall p^{\mathfrak{D}} \in \mathbb{R}^{\mathfrak{D}} .
$$

For a given mesh $\mathcal{T}$, we classically know that the scheme (2.5) is well-posed if and only if we have $\beta_{\mathcal{T}}>0$; see for instance [18. Actually, since the problem is finite-dimensional, it is easily seen that $\beta_{\mathcal{T}}=0$ if and only if there exists a non-zero pressure mode $p^{\mathfrak{D}}$, such that $m\left(p^{\mathfrak{D}}\right)=0$ and $\nabla^{\mathcal{T}} p^{\mathfrak{D}}=0$. In that case, the couple 
$\left(\mathbf{v}^{\mathcal{T}}=0, p^{\mathfrak{D}}\right)$ is a non-trivial solution to 2.5 with a zero right-hand side, which proves that the scheme is not well-posed.

For a given family of meshes, such that $\operatorname{size}(\mathcal{T}) \rightarrow 0$, we know that the scheme is stable if and only if

$$
\liminf _{\operatorname{size}(\mathcal{T}) \rightarrow 0} \beta_{\mathcal{T}}>0
$$

Assuming this property, it is very easy to adapt the proof of the error estimates given in 26] to get a convergence result for our scheme without any stabilization term.

The aim of this paper is thus to investigate from a theoretical and numerical point of view, whether or not the stability condition (2.8) holds for various kinds of mesh families. We will see that the results depend on the particular geometry of the meshes, in particular, for non-conforming meshes, which is a case of particular interest for applying the DDFV method.

2.6.2. Reformulation as an eigenvalue problem. In this section, we describe a practical method for computing, on a given mesh, the discrete Inf-Sup constant (2.6) for the Stokes DDFV scheme. The key point is to relate the value of $\beta_{\mathcal{T}}$ to the eigenvalues of a suitable matrix.

To be more precise, let us define $N_{\mathcal{T}}=\operatorname{Card}(\mathcal{T}), N_{\mathfrak{D}}=\operatorname{Card}(\mathfrak{D})$ and denote by $\langle\cdot, \cdot\rangle$ the Euclidean inner product on the spaces $\mathbb{R}^{2 N_{\mathcal{T}}}$ and $\mathbb{R}^{N_{\mathcal{D}}}$ and $|\cdot|$ the associated Euclidean norms. We are going to rewrite (2.5) and (2.6) by means of the following matrices:

- The stiffness matrix $R_{\mathcal{T}} \in \mathcal{M}_{2 N_{\mathcal{T}}}(\mathbb{R})$ such that for any $\mathbf{u}^{\mathcal{T}} \in\left(\mathbb{R}^{2}\right)^{\mathcal{T}}$, we have:

$$
R_{\mathcal{T}} \mathbf{u}^{\mathcal{T}}=\left(\begin{array}{c}
\left(\left(-\frac{m_{\mathcal{K}}}{2} \operatorname{div}^{\mathcal{K}}\left(\nabla^{\mathfrak{D}} \mathbf{u}^{\mathcal{T}}\right)\right)_{\mathcal{K} \in \mathfrak{M}}\right) \\
\mathbf{u}^{\partial \mathfrak{M}} \\
\left(\left(-\frac{m_{\mathcal{K}^{*}}}{2} \operatorname{div}^{\mathcal{K}^{*}}\left(\nabla^{\mathfrak{D}} \mathbf{u}^{\mathcal{T}}\right)\right)_{\mathcal{K}^{*} \in \mathfrak{M}^{*}}\right) \\
\mathbf{u}^{\partial \mathfrak{M}^{*}}
\end{array}\right)
$$

We can see that $R_{\mathcal{T}}$ satisfies

$$
\left\langle R_{\mathcal{T}} \mathbf{u}^{\mathcal{T}}, \mathbf{v}^{\mathcal{T}}\right\rangle=\left(\nabla^{\mathfrak{D}} \mathbf{u}^{\mathcal{T}}: \nabla^{\mathfrak{D}} \mathbf{v}^{\mathcal{T}}\right)_{\mathfrak{D}}, \quad \forall \mathbf{u}^{\mathcal{T}} \in\left(\mathbb{R}^{2}\right)^{\mathcal{T}}, \forall \mathbf{v}^{\mathcal{T}} \in \mathbb{E}_{0}
$$

Moreover, $R_{\mathcal{T}}$ maps $\mathbb{E}_{0}$ into $\mathbb{E}_{0}$ and is symmetric definite positive on $\mathbb{E}_{0}$. Therefore, $R_{\mathcal{T}}^{-1}$ and $R_{\mathcal{T}}^{ \pm 1 / 2}$ are well-defined operators that map $\mathbb{E}_{0}$ into itself.

- The divergence matrix $B_{\mathcal{T}} \in \mathcal{M}_{N_{\mathfrak{D}}, 2 N_{\mathcal{T}}}(\mathbb{R})$ such that for any $\mathbf{u}^{\mathcal{T}} \in \mathbb{E}_{0}$, $p^{\mathfrak{D}} \in \mathbb{R}^{\mathfrak{D}}$, we have

$$
\left\langle B_{\mathcal{T}} \mathbf{u}^{\mathcal{T}}, p^{\mathfrak{D}}\right\rangle=b_{\mathcal{T}}\left(\mathbf{u}^{\mathcal{T}}, p^{\mathfrak{D}}\right)
$$

We can see that

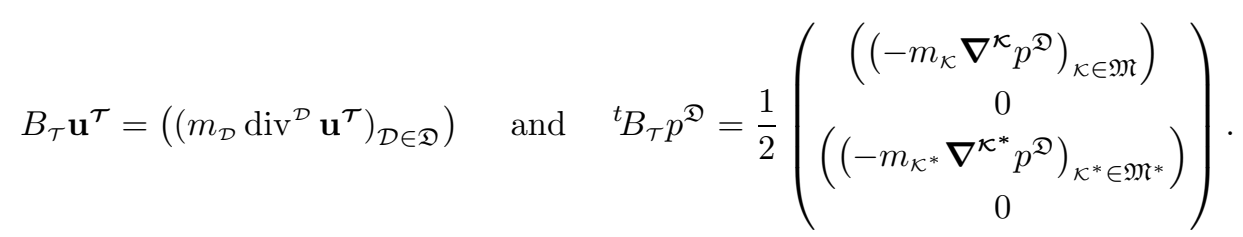

Observe that, by construction, ${ }^{t} B_{\mathcal{T}} p^{\mathfrak{D}} \in \mathbb{E}_{0}$ for any $p^{\mathfrak{D}} \in \mathbb{R}^{\mathfrak{D}}$. 
- The pressure mass matrix $M_{\mathcal{T}} \in \mathcal{M}_{N_{\mathfrak{D}}}(\mathbb{R})$ such that for any $p^{\mathfrak{D}}, q^{\mathfrak{D}} \in \mathbb{R}^{\mathfrak{D}}$, we have

$$
\left\langle M_{\mathcal{T}} p^{\mathfrak{D}}, q^{\mathfrak{D}}\right\rangle=\left(p^{\mathfrak{D}}, q^{\mathfrak{D}}\right)_{\mathfrak{D}}
$$

The matrix formulation of the scheme (2.5) is then: Find $\mathbf{u}^{\mathcal{T}} \in\left(\mathbb{R}^{2}\right)^{\mathcal{T}}$ and $p^{\mathfrak{D}} \in \mathbb{R}^{\mathfrak{D}}$ such that

$$
\left\{\begin{aligned}
\left(\begin{array}{cc}
R_{\mathcal{T}} & { }^{t} B_{\mathcal{T}} \\
B_{\mathcal{T}} & 0
\end{array}\right)\left(\begin{array}{c}
\mathbf{u}^{\mathcal{T}} \\
p^{\mathfrak{D}}
\end{array}\right) & =\left(\begin{array}{c}
\mathbb{P}_{\mathbf{m}}^{\mathcal{T}} \mathbf{f} \\
0
\end{array}\right) \\
\left\langle M_{\mathcal{T}} p^{\mathfrak{D}}, \mathbf{1}\right\rangle & =0 .
\end{aligned}\right.
$$

Using the matrices defined above we can now write (2.6) as follows:

$$
\beta_{\mathcal{T}}=\inf _{\substack{p^{\mathfrak{D}} \in \mathbb{R}^{\mathcal{D}} \\\left\langle M_{\mathcal{T}} p^{\mathfrak{D}}, \mathbf{1}\right\rangle=0}}\left(\sup _{\mathbf{v}^{\mathcal{T}} \in \mathbb{E}_{0}} \frac{\left\langle B_{\mathcal{T}} \mathbf{v}^{\mathcal{T}}, p^{\mathfrak{D}}\right\rangle}{\left\langle R_{\mathcal{T}} \mathbf{v}^{\mathcal{T}}, \mathbf{v}^{\mathcal{T}}\right\rangle^{\frac{1}{2}}\left\langle M_{\mathcal{T}} p^{\mathfrak{D}}, p^{\mathfrak{D}}\right\rangle^{\frac{1}{2}}}\right) .
$$

We show in the following lemma that, for a given mesh $\mathcal{T}$, computing $\beta_{\mathcal{T}}$ corresponds to solving a suitable eigenvalue problem (see [28] and [9, Section II.3]). We then solve this problem by using the subspace iteration method with Rayleigh-Ritz projections (see for instance [30]). We are then able to compute the actual value of $\beta_{\mathcal{T}}$ for different meshes and thus to investigate the Inf-Sup stability properties of the DDFV scheme.

Notation. From now on, for any square real matrix $M$ with real eigenvalues, we define $\lambda_{i}(M)$ to be the ith smallest eigenvalue of $M$.

Lemma 2.7 (Relation with the Schur complement). The discrete Inf-Sup constant $\beta_{\mathcal{T}}$ satisfies $\beta_{\mathcal{T}}^{2}=\lambda_{2}\left(S_{\mathcal{T}}\right)$, where $S_{\mathcal{T}}$ is the symmetric matrix defined by

$$
S_{\mathcal{T}}=M_{\mathcal{T}}^{-\frac{1}{2}} B_{\mathcal{T}} R_{\mathcal{T}}^{-1 t} B_{\mathcal{T}} M_{\mathcal{T}}^{-\frac{1}{2}} \in \mathcal{M}_{N_{\mathfrak{D}}}(\mathbb{R}) .
$$

Remark 2.8. If we set $p^{\mathfrak{D}}=M_{\mathcal{T}}^{\frac{1}{2}} \mathbf{1}$, we have $S_{\mathcal{T}} p^{\mathfrak{D}}=0$, so that the smallest eigenvalue of the matrix $S_{\mathcal{T}}$ is always $\lambda_{1}\left(S_{\mathcal{T}}\right)=0$.

Proof. We perform the change of variable $\mathbf{u}^{\mathcal{T}}=R_{\mathcal{\tau}}^{\frac{1}{2}} \mathbf{v}^{\mathcal{T}} \in \mathbb{E}_{0}$ in (2.10) and we get

$$
\begin{aligned}
\beta_{\mathcal{T}}= & \inf _{\substack{p^{\mathfrak{D}} \in \mathbb{R}^{\mathcal{D}} \\
\left\langle M_{\mathcal{T}} p^{\mathfrak{D}}, \mathbf{1}\right\rangle=0}}\left(\sup _{\mathbf{u}^{\mathcal{T} \in \mathbb{E}_{0}}} \frac{\left\langle B_{\mathcal{T}} R_{\mathcal{T}}^{-\frac{1}{2}} \mathbf{u}^{\mathcal{T}}, p^{\mathfrak{D}}\right\rangle}{\left\langle\mathbf{u}^{\mathcal{T}}, \mathbf{u}^{\mathcal{T}}\right\rangle^{\frac{1}{2}}\left\langle M_{\mathcal{T}} p^{\mathfrak{D}}, p^{\mathfrak{D}}\right\rangle^{\frac{1}{2}}}\right) \\
= & \inf _{\substack{p^{\mathfrak{D}} \in \mathbb{R}^{\mathcal{D}} \\
\left\langle M_{\mathcal{T}} p^{\mathcal{D}}, \mathbf{1}\right\rangle=0}} \frac{1}{\left\langle M_{\mathcal{T}} p^{\mathfrak{D}}, p^{\mathfrak{D}}\right\rangle^{\frac{1}{2}}}\left(\sup _{\mathbf{u}^{\mathcal{T} \in \mathbb{E}_{0}}} \frac{\left\langle\mathbf{u}^{\mathcal{T}}, R_{\mathcal{T}}^{-\frac{1}{2} t} B_{\mathcal{T}} p^{\mathfrak{D}}\right\rangle}{\left|\mathbf{u}^{\mathcal{T}}\right|}\right) \\
= & \inf _{\substack{\mathfrak{D} \in \mathbb{R}^{\mathcal{D}} \\
\left\langle M_{\mathcal{T}} p^{\mathfrak{D}}, \mathbf{1}\right\rangle=0}} \frac{\left|R_{\mathcal{T}}^{-\frac{1}{2} t} B_{\mathcal{T}} p^{\mathfrak{D}}\right|}{\left\langle M_{\mathcal{T}} p^{\mathfrak{D}}, p^{\mathfrak{D}}\right\rangle^{\frac{1}{2}}} .
\end{aligned}
$$

Considering now $\beta_{\mathcal{T}}^{2}$ and performing the change of variable $q^{\mathfrak{D}}=M_{\mathcal{T}}^{\frac{1}{2}} p^{\mathfrak{D}}$, we get

$$
\beta_{\mathcal{T}}^{2}=\inf _{\substack{\mathfrak{D} \in \mathbb{R}^{\mathfrak{D}} \\\left\langle M_{\mathcal{T}} p^{\mathfrak{P}}, \mathbf{1}\right\rangle=0}} \frac{\left\langle B_{\mathcal{T}} R_{\mathcal{T}}^{-1 t} B_{\mathcal{T}} p^{\mathfrak{D}}, p^{\mathfrak{D}}\right\rangle}{\left\langle M_{\mathcal{T}}^{\frac{1}{2}} p^{\mathfrak{D}}, M_{\mathcal{T}}^{\frac{1}{2}} p^{\mathfrak{D}}\right\rangle}=\inf _{\substack{q^{\mathfrak{D}} \in \mathbb{R}^{\mathfrak{D}} \\\left\langle M_{\mathcal{T}}^{\frac{1}{2}} q^{\mathfrak{D}}, \mathbf{1}\right\rangle=0}} \frac{\left\langle S_{\mathcal{T}} q^{\mathfrak{D}}, q^{\mathfrak{D}}\right\rangle}{\left|q^{\mathfrak{D}}\right|^{2}} .
$$


Thanks to Remark 2.8, we have actually proved that $\beta_{\mathcal{T}}^{2}$ is the second smallest eigenvalue of the matrix $S_{\mathcal{T}}$.

\section{Mesh families With unCONDitional InF-Sup Stability}

In this section, we study three families of meshes of the unit square domain $\Omega=] 0,1\left[^{2}\right.$ for which we are able to prove the unconditional Inf-Sup stability of the Stokes-DDFV scheme.

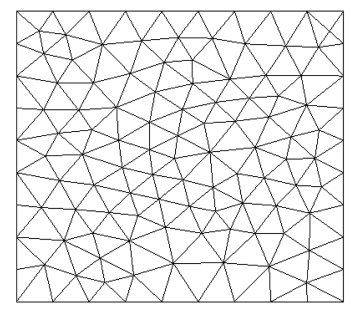

(a) Conforming triangle mesh

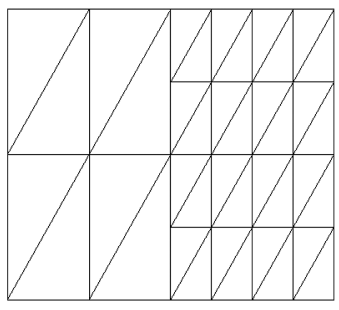

(b) Non-conforming triangle mesh

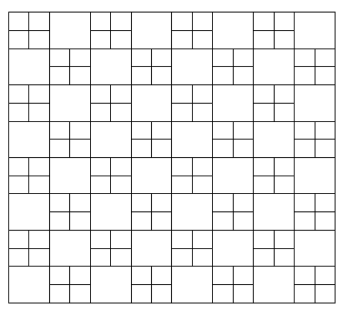

(c) Checkerboard mesh

FiguRE 3. First series of meshes

- The conforming triangle meshes on Figure 3a; note that all the results concerning this mesh family hold for any other connected polygonal domain $\Omega$.

- The non-conforming triangle mesh on Figure 3b, these meshes are obtained by performing a $k \times k$ rectangle mesh of the subdomain $] 0,0.5[\times] 0,1[$ and a $2 k \times 2 k$ rectangle mesh of the subdomain $] 0.5,1[\times] 0,1[$ then by dividing each rectangle into two triangles. We obtain a non-conforming triangle mesh. Note that the non-conforming edges are situated along one single line, called the interface.

- The checkerboard mesh on Figure $3 \mathrm{c}$ we start from a uniform square mesh of $\Omega$ then we divide half of the initial squares into 4 smaller squares as shown in the figure. This gives a non-conforming quadrangle mesh. Note that, contrary to the previous case, there are many non-conforming edges in this mesh (almost a constant proportion of the total number of edges).

3.1. Numerical results. For each of the three mesh families described above, we compute numerically the square root of the second smaller eigenvalue of $S_{\mathcal{T}}$ obtained by the subspace iteration method with Rayleigh-Ritz projection (see [30]).

We observe in each case the behavior of $\beta_{\mathcal{T}}=\sqrt{\lambda_{2}\left(S_{\mathcal{T}}\right)}$ as a function of the mesh size $\operatorname{size}(\mathcal{T})$ (see Figure 4).

We infer from these numerical experiments, that the DDFV scheme seems to be Inf-Sup stable for these mesh families, since we observe that $\beta_{\mathcal{T}}$ remains away from zero when $\operatorname{size}(\mathcal{T})$ goes to 0 . 


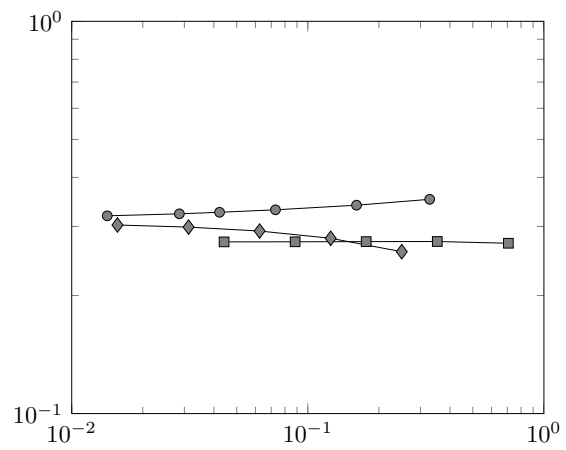

Conforming triangle meshes, Figure $3 \mathrm{a}-\mathrm{-}$ Non-conforming triangle meshes, Figure $3 \mathrm{~b}-\square$ Checkerboard meshes, Figure $3 \mathrm{C}-\diamond$

FiguRE 4. Stability investigation for a first series of meshes; $\beta_{\mathcal{T}}$ as a function of $\operatorname{size}(\mathcal{T})$

3.2. Theoretical results. In this section, we prove that the stability observed numerically in Figure 4 actually holds for these kind of meshes.

The analysis is based on the general theorem that we give below. It relies on the following property which is proved in [26, Proposition 5.5]. It consists in proving that the projection operator $\mathbb{P}_{\boldsymbol{m}}^{\mathcal{T}}$ is, in some sense, almost a Fortin operator.

Proposition 3.1. Let $\mathcal{T}$ be a DDFV mesh associated with $\Omega$. There exists a constant $C_{2}>0$, which depends only on $\operatorname{reg}(\mathcal{T})$, such that for any $\mathbf{v} \in\left(H_{0}^{1}(\Omega)\right)^{2}$ and $p^{\mathfrak{D}} \in \mathbb{R}^{\mathfrak{D}}$, we have

$$
\left|\sum_{\mathcal{D} \in \mathfrak{D}} \int_{\mathcal{D}} p^{\mathcal{D}}\left(\operatorname{div}^{\mathcal{D}} \mathbf{v}^{\mathcal{T}}-\operatorname{div} \mathbf{v}\right) \mathrm{d} z\right| \leq q_{2}\left|p^{\mathfrak{D}}\right|_{h}\|\mathbf{v}\|_{H^{1}}
$$

where $\mathbf{v}^{\mathcal{T}}=\mathbb{P}_{\boldsymbol{m}}^{\mathcal{T}} \mathbf{v}$ is the mean-value projection of $\mathbf{v}$ on the mesh $\mathcal{T}$ (see (2.3) ).

In this result, the following weak seminorm $|\cdot|_{h}$ over $\mathbb{R}^{\mathfrak{D}}$ is defined by

$$
\left|p^{\mathfrak{D}}\right|_{h}^{2}=\sum_{\substack{\mathcal{D}, \mathcal{D}^{\prime} \in \mathfrak{D} \\ \mathcal{D} \mid \mathcal{D}^{\prime}}}\left(h_{\mathcal{D}}^{2}+h_{\mathcal{D}^{\prime}}^{2}\right)\left(p^{\mathcal{D}^{\prime}}-p^{\mathcal{D}}\right)^{2}, \quad \forall p^{\mathfrak{D}} \in \mathbb{R}^{\mathfrak{D}}
$$

where the notation $\mathcal{D} \mid \mathcal{D}^{\prime}$ means that $\mathcal{D}$ and $\mathcal{D}^{\prime}$ have a common side; we say that they are neighbors.

Theorem 3.2 (General Inf-Sup stability result). Let be $\mathcal{T}$ a DDFV mesh on a connected polygonal domain $\Omega$.

Assume that there is $\alpha_{\mathcal{T}} \geq 1$ such that

$$
\left|p^{\mathfrak{D}}\right|_{h} \leq \alpha_{\mathcal{T}}\left\|h^{\mathcal{T}} \nabla^{\mathcal{T}} p^{\mathfrak{D}}\right\|_{\mathcal{T}, 2}, \quad \forall p^{\mathfrak{D}} \in \mathbb{R}^{\mathfrak{D}} .
$$

Then, there exists $C_{3}>0$ depending only on $\operatorname{reg}(\mathcal{T})$ such that

$$
\frac{G 3}{\alpha_{\mathcal{T}}}\left\|p^{\mathfrak{D}}-m\left(p^{\mathfrak{D}}\right)\right\|_{\mathfrak{D}, 2} \leq \sup _{\mathbf{v} \mathcal{\mathcal { T }} \in \mathbb{E}_{0}} \frac{b_{\mathcal{T}}\left(\mathbf{v}^{\mathcal{T}}, p^{\mathfrak{D}}\right)}{\left\|\nabla^{\mathfrak{D}} \mathbf{v}^{\mathcal{T}}\right\|_{\mathfrak{D}, 2}}, \quad \forall p^{\mathfrak{D}} \in \mathbb{R}^{\mathfrak{D}},
$$

that is, $\beta_{\mathcal{T}} \geq 9 \sqrt[3]{3} / \alpha_{\mathcal{T}}$.

As consequence, if we consider a regular mesh family (that is such that $\operatorname{reg}(\mathcal{T})$ is bounded when $\operatorname{size}(\mathcal{T}) \rightarrow 0$ ), then the Inf-Sup stability of the scheme will be proved if we can obtain the norm equivalence property (3.3) with a number $\alpha_{\mathcal{T}}$ which is 
bounded as soon as $\operatorname{size}(\mathcal{T}) \rightarrow 0$. Proving this last property will be, for instance, the aim of Propositions 3.3 3.5 and 3.6 for different kind of mesh families.

Proof. Let us consider a fixed $p^{\mathfrak{D}} \in \mathbb{R}^{\mathfrak{D}}$. By adding a constant to $p^{\mathfrak{D}}$, it is clear that we can always assume that $m\left(p^{\mathfrak{D}}\right)=0$.

Let $C_{2}>0$ be the constant appearing in (3.1) and $\Pi$ be the continuous rightinverse of the divergence, as introduced in Proposition 1.1

We divide the analysis into two cases in a similar way as in the classical proof of the Inf-Sup stability of the Taylor-Hood element (see [18, 22]).

- First case, we assume that $p^{\mathfrak{D}}$ is such that

$$
\left\|h^{\mathcal{T}} \nabla^{\mathcal{T}} p^{\mathfrak{D}}\right\|_{\mathcal{T}, 2} \leq \frac{1}{2\|\Pi\| C_{2}{ }^{\alpha_{\mathcal{T}}}}\left\|p^{\mathfrak{D}}\right\|_{\mathfrak{D}, 2} .
$$

Since the integral of the piecewise constant function $p^{\mathfrak{D}}=\sum_{\mathcal{D} \in \mathfrak{D}} p^{\mathcal{D}} \mathbf{1}_{\mathcal{D}} \in$ $L^{2}(\Omega)$ is equal to zero on $\Omega$, we can take $\mathbf{v}=\Pi\left(p^{\mathfrak{D}}\right)$ so that

$$
\operatorname{div} \mathbf{v}=p^{\mathfrak{D}} \quad \text { and } \quad\|\mathbf{v}\|_{H^{1}} \leq\|\Pi\|\left\|p^{\mathfrak{D}}\right\|_{\mathfrak{D}, 2} .
$$

We set now $\mathbf{v}^{\mathcal{T}}=\mathbb{P}_{\boldsymbol{m}}^{\mathcal{T}} \mathbf{v}$ so that, by construction, we have $\mathbf{v}^{\mathcal{T}} \in \mathbb{E}_{0}$. By using (2.4), we obtain

$$
\left\|\nabla^{\mathfrak{D}} \mathbf{v}^{\mathcal{T}}\right\|_{\mathfrak{D}, 2} \leq \mathcal{Q}_{\mathfrak{1}}\|\mathbf{v}\|_{H^{1}} \leq\|\Pi\| \mathcal{G}_{1}\left\|p^{\mathfrak{D}}\right\|_{\mathfrak{D}, 2}
$$

We add and subtract $\sum_{\mathcal{D} \in \mathfrak{D}} \int_{\mathcal{D}} p^{\mathcal{D}} \operatorname{div} \mathbf{v}$ to $b_{\mathcal{T}}\left(\mathbf{v}^{\mathcal{T}}, p^{\mathfrak{D}}\right)$ and we use (3.6) to get

$$
b_{\mathcal{T}}\left(\mathbf{v}^{\mathcal{T}}, p^{\mathfrak{D}}\right)=\left\|p^{\mathfrak{D}}\right\|_{\mathfrak{D}, 2}^{2}+\sum_{\mathcal{D} \in \mathfrak{D}} \int_{\mathcal{D}} p^{\mathcal{D}}\left(\operatorname{div}^{\mathcal{D}} \mathbf{v}^{\mathcal{T}}-\operatorname{div} \mathbf{v}\right)
$$

Proposition 3.1 and estimate (3.6) imply

$$
b_{\mathcal{T}}\left(\mathbf{v}^{\mathcal{T}}, p^{\mathfrak{D}}\right) \geq\left\|p^{\mathfrak{D}}\right\|_{\mathfrak{D}, 2}^{2}-\|\Pi\| C\left[{ }_{2}\left|p^{\mathfrak{D}}\right|_{h}\left\|p^{\mathfrak{D}}\right\|_{\mathfrak{D}, 2} .\right.
$$

We apply now the assumption (3.3),

$$
b_{\mathcal{T}}\left(\mathbf{v}^{\mathcal{T}}, p^{\mathfrak{D}}\right) \geq\left\|p^{\mathfrak{D}}\right\|_{\mathfrak{D}, 2}^{2}-\|\Pi\| C_{2} \alpha_{\mathcal{T}}\left\|h^{\mathcal{T}} \nabla^{\mathcal{T}} p^{\mathfrak{D}}\right\|_{\mathcal{T}, 2}\left\|p^{\mathfrak{D}}\right\|_{\mathfrak{D}, 2} .
$$

By assumption (3.5), it is now clear that we have

$$
b_{\mathcal{T}}\left(\mathbf{v}^{\mathcal{T}}, p^{\mathfrak{D}}\right) \geq \frac{1}{2}\left\|p^{\mathfrak{D}}\right\|_{\mathfrak{D}, 2}^{2}
$$

Thus, according to (3.7), letting be $\beta_{1}=\frac{1}{2\|\Pi\| \mathcal{C}_{1}}$, we finally obtain

$$
\beta_{1}\left\|p^{\mathfrak{D}}\right\|_{\mathfrak{D}, 2} \leq \sup _{\mathbf{v} \mathcal{T} \in \mathbb{E}_{0}} \frac{b_{\mathcal{T}}\left(\mathbf{v}^{\mathcal{T}}, p^{\mathfrak{D}}\right)}{\left\|\nabla^{\mathfrak{D}} \mathbf{v}^{\mathcal{T}}\right\|_{\mathfrak{D}, 2}}
$$

- Second case, we assume that $p^{\mathfrak{D}}$ is such that

$$
\left\|h^{\mathcal{T}} \nabla^{\mathcal{T}} p^{\mathfrak{D}}\right\|_{\mathcal{T}, 2} \geq \frac{1}{2\|\Pi\| C_{2}{ }^{\alpha_{\mathcal{T}}}}\left\|p^{\mathfrak{D}}\right\|_{\mathfrak{D}, 2} .
$$

In that case, we do not use the operator $\Pi$ but we directly build a $\mathbf{v}^{\mathcal{T}} \in \mathbb{E}_{0}$ as follows:

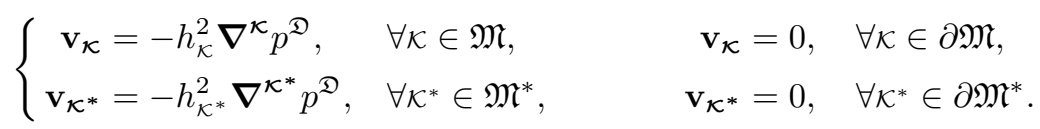


For any $\mathcal{D} \in \mathfrak{D}$, by using Definition 2.1 we have, for some $C>0$ depending only on $\operatorname{reg}(\mathcal{T})$,

$$
\begin{aligned}
m_{\mathcal{D}}\left|\nabla^{\mathcal{D}} \mathbf{v}^{\mathcal{T}}\right|^{2} \leq & C\left(\left|\mathbf{v}_{\mathcal{K}}-\mathbf{v}_{\mathcal{L}}\right|^{2}+\left|\mathbf{v}_{\mathcal{K}^{*}}-\mathbf{v}_{\mathcal{L}^{*}}\right|^{2}\right) \\
\leq & 2 C\left(\left|\mathbf{v}_{\mathcal{K}}\right|^{2}+\left|\mathbf{v}_{\mathcal{L}}\right|^{2}+\left|\mathbf{v}_{\mathcal{K}^{*}}\right|^{2}+\left|\mathbf{v}_{\mathcal{L}^{*}}\right|^{2}\right) \\
= & 2 C\left(\left|h_{\mathcal{K}}^{2} \nabla^{\mathcal{K}} p^{\mathfrak{D}}\right|^{2}+\left|h_{\mathcal{L}}^{2} \nabla^{\mathcal{L}} p^{\mathfrak{D}}\right|^{2}+\left|h_{\mathcal{K}^{*}}^{2} \nabla^{\mathcal{K}^{*}} p^{\mathfrak{D}}\right|^{2}+\left|h_{\mathcal{L}^{*}}^{2} \nabla^{\mathcal{L}^{*}} p^{\mathfrak{D}}\right|^{2}\right) \\
\leq & 2 C \operatorname{reg}(\mathcal{T})^{2}\left(m_{\mathcal{K}}\left|h_{\mathcal{K}} \boldsymbol{\nabla}^{\mathcal{K}} p^{\mathfrak{D}}\right|^{2}+m_{\mathcal{L}}\left|h_{\mathcal{L}} \nabla^{\mathcal{L}} p^{\mathfrak{D}}\right|^{2}\right. \\
& \left.\quad+m_{\mathcal{K}^{*}}\left|h_{\mathcal{K}^{*}} \nabla^{\mathcal{K}^{*}} p^{\mathfrak{D}}\right|^{2}+m_{\mathcal{L}^{*}}\left|h_{\mathcal{L}^{*}} \nabla^{\mathcal{L}^{*}} p^{\mathfrak{D}}\right|^{2}\right) .
\end{aligned}
$$

It follows that, for some $C_{4}>0$ depending only on $\operatorname{reg}(\mathcal{T})$, we have

$$
\left\|\nabla^{\mathfrak{D}} \mathbf{v}^{\mathcal{T}}\right\|_{\mathfrak{D}, 2} \leq \mathcal{C}_{4}\left\|h^{\mathcal{T}} \nabla^{\mathcal{T}} p^{\mathfrak{D}}\right\|_{\mathcal{T}, 2}
$$

Moreover, by (3.9) we get

$$
b_{\mathcal{T}}\left(\mathbf{v}^{\mathcal{T}}, p^{\mathfrak{D}}\right)=-\llbracket \mathbf{v}^{\mathcal{T}}, \nabla^{\mathcal{T}} p^{\mathfrak{D}} \rrbracket_{\mathcal{T}}=\left\|h^{\mathcal{T}} \nabla^{\mathcal{T}} p^{\mathfrak{D}}\right\|_{\mathcal{T}, 2}^{2} .
$$

Thus, we conclude with $\beta_{2}=\frac{1}{2 C_{4}\|\Pi\| C_{2}}$, that

$$
\frac{b_{\mathcal{T}}\left(\mathbf{v}^{\mathcal{T}}, p^{\mathfrak{D}}\right)}{\left\|\nabla^{\mathfrak{D}} \mathbf{v}^{\mathcal{T}}\right\|_{\mathfrak{D}, 2}} \geq \frac{1}{\mathcal{C}_{4}}\left\|h^{\mathcal{T}} \nabla^{\mathcal{T}} p^{\mathfrak{D}}\right\|_{\mathcal{T}, 2} \geq \frac{\beta_{2}}{\alpha_{\mathcal{T}}}\left\|p^{\mathfrak{D}}\right\|_{\mathfrak{D}, 2}
$$

Noting that $\alpha_{\mathcal{T}} \geq 1$ by definition, the claim is proved with $C_{3}=\min \left(\beta_{1}, \beta_{2}\right)$ which actually only depends on $\operatorname{reg}(\mathcal{T})$.

Our strategy now is to investigate whether or not the inequality (3.3) holds for some $\alpha_{\mathcal{T}}$ which is uniform with respect to $\operatorname{size}(\mathcal{T})$ for each of the mesh families described above. It corresponds to an estimate of differences of two pressure values on neighboring diamond cells in terms of the DDFV pressure gradient which is defined on primal and dual meshes. The difficulty comes from the fact that the value of the pressure gradient on a given cell depends on the pressure values on all the diamond cells associated with that cell. Therefore, it is not necessarily easy to deduce an estimate on the difference of two pressure values.

In order to perform this analysis, we introduce the following notations.

Notations. Let $\mathcal{K}$ be a primal cell and $\mathcal{D}, \mathcal{D}^{\prime}, \mathcal{D}^{\prime \prime}$ three diamond cells of $\mathcal{K}$.

- We say that $p^{\mathcal{D}} \stackrel{\mathcal{K}}{\longrightarrow} p^{\mathcal{D}^{\prime}}$ if there is a $C$ depending only on $\operatorname{reg}(\mathcal{T})$ such that

$$
\left|p^{\mathcal{D}}-p^{\mathcal{D}^{\prime}}\right| \leq C \frac{m_{\mathcal{K}}}{h_{\mathcal{K}}}\left|\nabla^{\mathcal{K}} p^{\mathfrak{D}}\right| .
$$

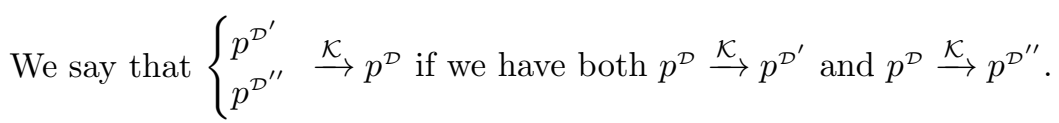

- We say that $p^{\mathcal{D}} \stackrel{\mathcal{K}}{\rightarrow}\left\{\begin{array}{l}p^{\mathcal{D}^{\prime}} \\ p^{\mathcal{D}^{\prime \prime}}\end{array}\right.$ if there exists a $C$ depending only on $\operatorname{reg}(\mathcal{T})$ and a $\theta \in[0,1]$ such that

$$
\left|\theta\left(p^{\mathcal{D}}-p^{\mathcal{D}^{\prime}}\right)+(1-\theta)\left(p^{\mathcal{D}}-p^{\mathcal{D}^{\prime \prime}}\right)\right| \leq C \frac{m_{\mathcal{K}}}{h_{\mathcal{K}}}\left|\nabla^{\mathcal{K}} p^{\mathfrak{D}}\right| .
$$

- Similar notations are used for dual cells $\mathcal{K}^{*} \in \mathfrak{M}^{*}$ in place of primal cells $\mathcal{K} \in \mathfrak{M}$. 
Proposition 3.3 (The case of conforming triangle mesh). For a conforming triangle mesh (see Figure 3a), the inequality (3.3) holds with a $\alpha_{\mathcal{T}}$ which depends only on $\operatorname{reg}(\mathcal{T})$.

As a consequence, for a regular family of conforming triangle meshes, the DDFV scheme is Inf-Sup stable.

Proof. For any primal control volume $\mathcal{K}$, we have three diamond cells in $\mathfrak{D}_{\mathcal{K}}$ (one associated with each edge), that we note $\mathcal{D}_{1}, \mathcal{D}_{2}, \mathcal{D}_{3}$. By definition of the discrete pressure gradient we have

$$
m_{\mathcal{K}} \boldsymbol{\nabla}^{\mathcal{K}} p^{\mathfrak{D}}=\sum_{i=1}^{3} m_{\sigma_{i}} p^{\mathcal{D}_{i}} \overrightarrow{\mathbf{n}}_{\boldsymbol{\sigma}_{i} \mathcal{K}} \quad \text { and } \quad \sum_{i=1}^{3} m_{\sigma_{i}} \overrightarrow{\mathbf{n}}_{\boldsymbol{\sigma}_{i} \mathcal{K}}=0 .
$$

This implies, for instance,

$$
m_{\mathcal{K}} \boldsymbol{\nabla}^{\mathcal{K}} p^{\mathfrak{D}}=m_{\sigma_{1}}\left(p^{\mathcal{D}_{1}}-p^{\mathcal{D}_{3}}\right) \overrightarrow{\mathbf{n}}_{\sigma_{1} \mathcal{K}}+m_{\sigma_{2}}\left(p^{\mathcal{D}_{2}}-p^{\mathcal{D}_{3}}\right) \overrightarrow{\mathbf{n}}_{\sigma_{2} \mathcal{K}} .
$$

We use now the formula

$$
2 m_{\mathcal{K}}=\left|\left(m_{\sigma_{i}} \overrightarrow{\mathbf{n}}_{\sigma_{i} \mathcal{K}}\right) \wedge\left(m_{\sigma_{j}} \overrightarrow{\mathbf{n}}_{\sigma_{j} \mathcal{K}}\right)\right|, \quad \forall i \neq j,
$$

to get

$$
\left|p^{\mathcal{D}_{1}}-p^{\mathcal{D}_{3}}\right|=\frac{m_{\sigma_{2}}}{2}\left|\nabla^{\mathcal{K}} p^{\mathfrak{D}} \wedge \overrightarrow{\mathbf{n}}_{\sigma_{\mathbf{2}} \mathcal{K}}\right| \leq C \frac{m_{\mathcal{K}}}{h_{\mathcal{K}}}\left|\nabla^{\mathcal{K}} p^{\mathfrak{D}}\right| .
$$

We just proved that $p^{\mathcal{D}_{1}} \stackrel{\mathcal{K}}{\longrightarrow} p^{\mathcal{D}_{3}}$ and the same argument gives a similar estimate for the other two possible couples of diamond cells associated with $\mathcal{K}$.

Summing these estimates over the whole domain gives the result

$$
\begin{aligned}
\left|p^{\mathfrak{D}}\right|_{h}^{2} & =\sum_{\substack{\mathcal{D}, \mathcal{D}^{\prime} \in \mathfrak{D} \\
\mathcal{D} \mid \mathcal{D}^{\prime}}}\left(h_{\mathcal{D}}^{2}+h_{\mathcal{D}^{\prime}}^{2}\right)\left(p^{\mathcal{D}^{\prime}}-p^{\mathcal{D}}\right)^{2} \\
& \leq C(\operatorname{reg}(\mathcal{T})) \sum_{\mathcal{K} \in \mathfrak{M}} h_{\mathcal{K}}^{2} m_{\mathcal{K}}\left|\nabla^{\mathcal{K}} p^{\mathfrak{D}}\right|^{2} \leq C(\operatorname{reg}(\mathcal{T}))\left\|h^{\mathcal{T}} \nabla^{\mathcal{T}} p^{\mathfrak{D}}\right\|_{\mathcal{T}, 2}^{2} .
\end{aligned}
$$

Remark 3.4. Observe that, in this proof, we do not use the pressure gradient on the dual cells. Actually, the same proof is valid if we assume that the dual cells are conforming triangle cells.

Proposition 3.5 (The case of a non-conforming triangle mesh). For the nonconforming triangle mesh described in Figure 3b, the inequality (3.3) holds with a $\alpha_{\mathcal{T}}$, which depends only on $\operatorname{reg}(\mathcal{T})$.

As a consequence, for this particular family of non-conforming triangle meshes, the DDFV scheme is Inf-Sup stable.

Proof. We can see that if the control volume $\mathcal{K}$ has no edge on the interface or if $\mathcal{K}$ is of the right-hand side of the interface, we can apply the same proof as in the previous proposition.

Thus, the only case that we need to study carefully is the one of a primal cell $\mathcal{K}$ just on the left of the interface. Even though it is triangle-shaped, such a control volume is rather a degenerated quadrangle cell since it has 4 neighboring cells and thus 4 edges and 4 associated diamond cells. That is the reason why the situation is different, and more complicated, than in the previous proposition.

The situation under study is described in Figure 5. We need to bound all the possible neighboring pressure differences in $\mathcal{K}$ (that is $p^{\mathcal{D}_{1}}-p^{\mathcal{D}_{2}}, p^{\mathcal{D}_{1}}-p^{\mathcal{D}_{4}}, p^{\mathcal{D}_{2}}-p^{\mathcal{D}_{3}}$ and $\left.p^{\mathcal{D}_{3}}-p^{\mathcal{D}_{4}}\right)$ by some quantity depending only on values of the pressure gradient 


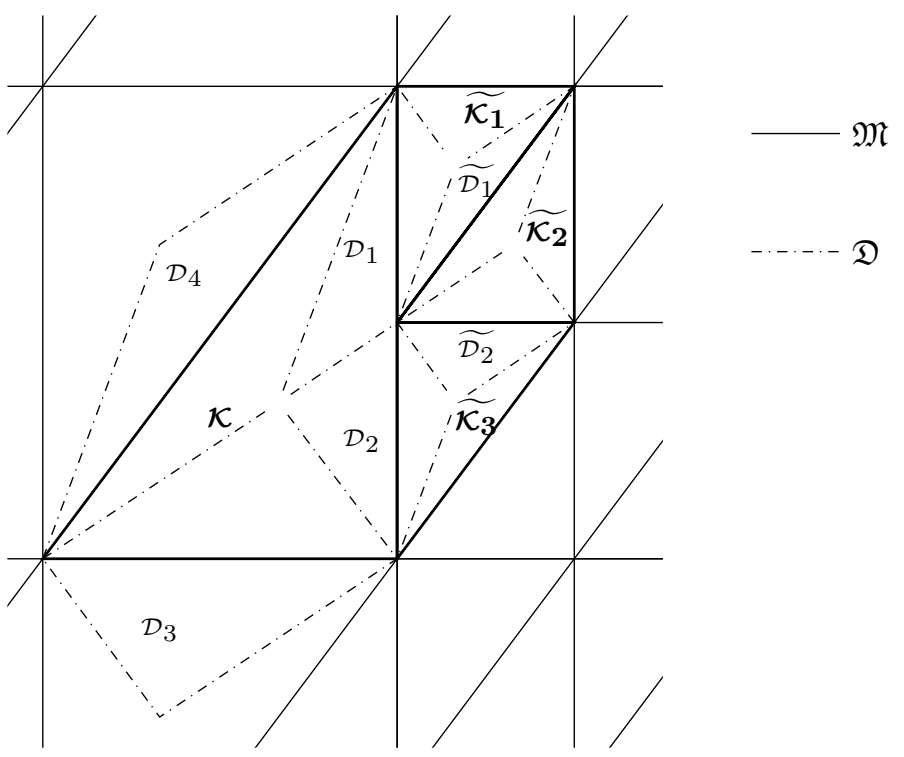

FiguRE 5. Notations near the interface for the mesh $3 \mathrm{~b}$

on a few (fixed) number of cells. Here, we will absolutely need to use neighboring primal cells in order to get the result. Indeed, the pressure difference $p^{\mathcal{D}_{1}}-p^{\mathcal{D}_{2}}$ for instance cannot been estimated by simply using the pressure gradient on the given primal cell $\mathcal{K}$. Indeed, if $p^{\mathcal{D}_{3}}=p^{\mathcal{D}_{4}}=0$ and $p^{\mathcal{D}_{1}}=-\frac{m_{\sigma_{2}}}{m_{\sigma_{1}}} p^{\mathcal{D}_{2}} \neq 0$, then $\nabla^{\mathcal{K}} p^{\mathfrak{D}}$ is zero whereas $p^{\mathcal{D}_{1}}-p^{\mathcal{D}_{2}} \neq 0$.

- We start by proving a bound on $p^{\mathcal{D}_{1}}-p^{\mathcal{D}_{2}}$ by using the pressure gradients on the other primal control volumes around $\mathcal{K}$. We can see on Figure 5 , and using the same argument as in the proof of Proposition 3.3. that the following chain holds:

$$
p^{\mathcal{D}_{1}} \stackrel{\widetilde{\mathcal{K}_{1}}}{\longrightarrow} p^{\widetilde{\mathcal{D}_{1}}} \stackrel{\widetilde{\mathcal{K}_{2}}}{\longrightarrow} p^{\widetilde{\mathcal{D}_{2}}} \stackrel{\widetilde{\mathcal{K}_{3}}}{\longrightarrow} p^{\mathcal{D}_{2}},
$$

so that, by the triangle inequality, we get that

$$
\left|p^{\mathcal{D}_{1}}-p^{\mathcal{D}_{2}}\right| \leq C\left(\frac{m_{\widetilde{\mathcal{K}_{1}}}}{h_{\widetilde{\mathcal{K}_{1}}}}\left|\nabla^{\widetilde{\mathcal{K}_{1}}} p^{\mathfrak{D}}\right|+\frac{m_{\widetilde{\mathcal{K}_{2}}}}{h_{\widetilde{\mathcal{K}_{2}}}}\left|\nabla^{\widetilde{\mathcal{K}_{2}}} p^{\mathfrak{D}}\right|+\frac{m_{\widetilde{\mathcal{K}_{3}}}}{h_{\widetilde{\mathcal{K}_{3}}}}\left|\nabla^{\widetilde{\mathcal{K}_{3}}} p^{\mathfrak{D}}\right|\right) .
$$

- We can now study the pressure gradient in the actual control volume under study $\mathcal{K}$ and use that $\overrightarrow{\mathbf{n}}_{\sigma_{1} \mathcal{K}}=\overrightarrow{\mathbf{n}}_{\sigma_{2} \mathcal{K}}$, so that

$$
\begin{gathered}
\left|p^{\mathcal{D}_{3}}-p^{\mathcal{D}_{4}}\right|=\frac{m_{\sigma_{1}}+m_{\sigma_{2}}}{2}\left|\nabla^{\mathcal{K}} p^{\mathfrak{D}} \wedge \overrightarrow{\mathbf{n}}_{\sigma_{1} \mathcal{K}}\right| \leq C \frac{m_{\mathcal{K}}}{h_{\mathcal{K}}}\left|\nabla^{\mathcal{K}} p^{\mathfrak{D}}\right|, \\
\left|p^{\mathcal{D}_{1}}-p^{\mathcal{D}_{4}}\right| \leq \frac{m_{\sigma_{3}}}{2}\left|\nabla^{\mathcal{K}} p^{\mathfrak{D}} \wedge \overrightarrow{\mathbf{n}}_{\sigma_{3} \mathcal{K}}\right|+\left|p^{\mathcal{D}_{1}}-p^{\mathcal{D}_{2}}\right| \leq C \frac{m_{\mathcal{K}}}{h_{\mathcal{K}}}\left|\nabla^{\mathcal{K}} p^{\mathfrak{D}}\right|+\left|p^{\mathcal{D}_{1}}-p^{\mathcal{D}_{2}}\right|, \\
\left|p^{\mathcal{D}_{2}}-p^{\mathcal{D}_{3}}\right| \leq \frac{m_{\sigma_{4}}}{2}\left|\nabla^{\mathcal{K}} p^{\mathfrak{D}} \wedge \overrightarrow{\mathbf{n}}_{\sigma_{4} \mathcal{K}}\right|+\left|p^{\mathcal{D}_{1}}-p^{\mathcal{D}_{2}}\right| \leq C \frac{m_{\mathcal{K}}}{h_{\mathcal{K}}}\left|\nabla^{\mathcal{K}} p^{\mathfrak{D}}\right|+\left|p^{\mathcal{D}_{1}}-p^{\mathcal{D}_{2}}\right| .
\end{gathered}
$$

Combining all the estimates above, we see that any difference between two neighboring pressure values can be bounded by using at most 4 neighboring values of the primal pressure gradient, and we can conclude the proof as in the previous proposition. 
Finally, we are able to prove the same Inf-Sup stability property for highly nonconforming meshes, that is to say for some meshes containing a constant proportion of non-conforming edges. This result seems to show that the Inf-Sup stability of the DDFV method is very robust with respect to the non-conformity of the mesh.

Proposition 3.6 (The checkerboard mesh). For a checkerboard mesh as described in Figure 3c, the inequality (3.3) holds with a $\alpha_{\mathcal{T}}$ which does not depend on $\operatorname{size}(\mathcal{T})$.

As a consequence, the DDFV scheme is Inf-Sup stable for this particular family of non-conforming meshes.

$\partial \Omega$

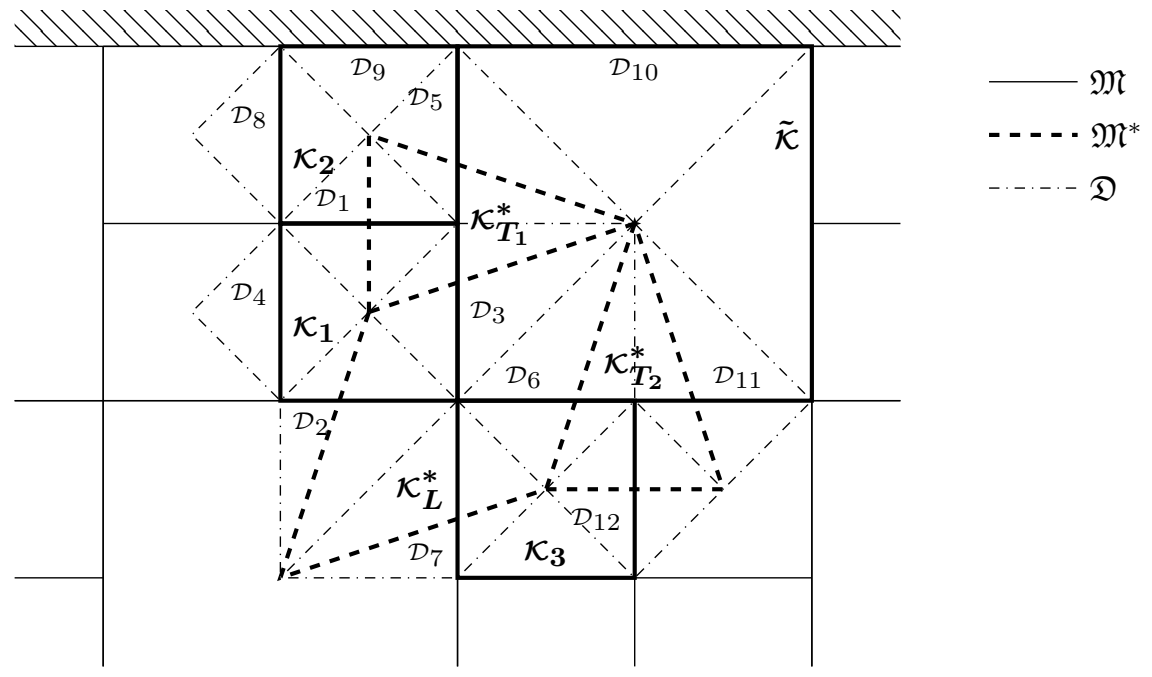

Figure 6. Possible configurations of neighboring diamond cells for the checkerboard mesh; see Figure $3 \mathrm{c}$

Proof. Let us begin with some remarks. First, the smallest primal cells are square for which it is impossible to bound all the possible associated pressure differences by simply using the primal pressure gradient (since the pressure mode where two opposite pressures equal 1 and the other two equal -1 clearly has a zero pressure gradient). Second, the biggest primal cells are degenerate octagons since they are associated with 8 different edges/diamond cells. These two reasons make the analysis quite difficult.

All the generic configurations of neighboring diamond cells are presented in Figure 6 .

- Let us first look at the situation away from the boundary. Using that $\mathcal{K}_{1}$ is a square primal control volume, we deduce

$$
p^{\mathcal{D}_{1}} \stackrel{\mathcal{K}_{1}}{\longrightarrow} p^{\mathcal{D}_{2}} \text { and } p^{\mathcal{D}_{3}} \stackrel{\mathcal{K}_{1}}{\longrightarrow} p^{\mathcal{D}_{4}} .
$$

Using that $\mathcal{K}_{T_{1}}^{*}$ is a triangle dual control volume we deduce

$$
p^{\mathcal{D}_{1}} \stackrel{\mathcal{K}_{T_{1}}^{*}}{\longrightarrow} p^{\mathcal{D}_{3}}, \quad p^{\mathcal{D}_{1}} \stackrel{\mathcal{K}_{T_{1}}^{*}}{\longrightarrow} p^{\mathcal{D}_{5}} \text { and } p^{\mathcal{D}_{3}} \stackrel{\mathcal{K}_{T_{1}}^{*}}{\longrightarrow} p^{\mathcal{D}_{5}} .
$$


Finally, we use that $\mathcal{K}_{L}^{*}$ is a parallelogram. Just like in a square, the difference between opposite pressures can be controlled by the pressure gradient, so that

$$
p^{\mathcal{D}_{2}} \stackrel{\mathcal{K}_{L}^{*}}{\longrightarrow} p^{\mathcal{D}_{6}} \text { and } p^{\mathcal{D}_{3}} \stackrel{\mathcal{K}_{L}^{*}}{\longrightarrow} p^{\mathcal{D}_{7}} .
$$

We can combine the previous arrows to obtain that

$$
\begin{array}{ll}
p^{\mathcal{D}_{1}} \stackrel{\mathcal{K}_{T_{1}}^{*}}{\longrightarrow} p^{\mathcal{D}_{3}} \stackrel{\mathcal{K}_{1}}{\longrightarrow} p^{\mathcal{D}_{4}}, & p^{\mathcal{D}_{1}} \stackrel{\mathcal{K}_{T_{1}}^{*}}{\longrightarrow} p^{\mathcal{D}_{5}} \stackrel{\mathcal{K}_{2}}{\longrightarrow} p^{\mathcal{D}_{8}}, \\
p^{\mathcal{D}_{3}} \stackrel{\mathcal{K}_{T_{1}}^{*}}{\longrightarrow} p^{\mathcal{D}_{1}} \stackrel{\mathcal{K}_{1}}{\longrightarrow} p^{\mathcal{D}_{2}}, & p^{\mathcal{D}_{3}} \stackrel{\mathcal{K}_{T_{1}}^{*}}{\longrightarrow} p^{\mathcal{D}_{1}} \stackrel{\mathcal{K}_{1}}{\longrightarrow} p^{\mathcal{D}_{2}} \stackrel{\mathcal{K}_{L}^{*}}{\longrightarrow} p^{\mathcal{D}_{6}} .
\end{array}
$$

Using the triangle inequality, and the symmetry properties of the mesh, we can see that all possible pressure differences between neighboring interior diamond cells can be estimated by the previous computations. Notice that some estimates require both primal and dual pressure gradient.

- It remains to cope with the case of boundary diamond cells, since we recall that the dual pressure gradient is conventionally set to 0 on boundary dual cells (see Remark 2.5) and therefore it cannot be used to obtain useful estimates.

We observe in Figure 6 two kinds of boundary diamond cells that need to be investigated.

- The terms involving the diamond cell $\mathcal{D}_{9}$ can be treated as in the interior case by using the primal pressure gradient on $\mathcal{K}_{2}$ and the dual pressure gradient on $\mathcal{K}_{T_{1}}^{*}$.

- Using symmetries of the mesh, we see that the only term involving the diamond cell $\mathcal{D}_{10}$ that has to be carefully studied is the term $p^{\mathcal{D}_{10}}-p^{\mathcal{D}_{5}}$ since the corresponding dual cell is a boundary dual cell for which the corresponding pressure gradient cannot be used.

We observe that, by definition of the dual pressure gradient on $\widetilde{\mathcal{K}}$, we have

$$
m_{\sigma_{10}}\left(p^{\mathcal{D}_{10}}-\frac{1}{2}\left(p^{\mathcal{D}_{6}}+p^{\mathcal{D}_{11}}\right)\right)=m_{\widetilde{\mathcal{K}}}\left(\nabla^{\widetilde{\mathcal{K}}} p^{\mathfrak{D}} \cdot \overrightarrow{\mathbf{n}}_{\sigma_{10} \widetilde{\mathcal{K}}}\right) .
$$

It follows that the following chain holds

$$
p^{\mathcal{D}_{10}} \stackrel{\widetilde{\mathcal{K}}}{\longrightarrow}\left\{\begin{array}{l}
p^{\mathcal{D}_{6}} \\
p^{\mathcal{D}_{11}}
\end{array} \stackrel{\mathcal{K}_{T_{2}}^{*}}{\longrightarrow} p^{\mathcal{D}_{12}} \stackrel{\mathcal{K}_{3}}{\longrightarrow} p^{\mathcal{D}_{7}} \stackrel{\mathcal{K}_{L}^{*}}{\longrightarrow} p^{\mathcal{D}_{3}} \stackrel{\mathcal{K}_{T_{1}}^{*}}{\longrightarrow} p^{\mathcal{D}_{5}},\right.
$$

and the proof is complete.

\section{Codimension 1 Inf-Sup Stability}

In this section, we shall study the stability properties of the Stokes DDFV scheme for two families of Cartesian meshes of the unit square domain: the usual uniform conforming meshes, and two-subdomain non-conforming Cartesian meshes (see Figure (7). Note that, the same analysis can be applied to other kinds of Cartesian meshes as we will show in Section 5.

In both cases, we prove (Theorems 4.2 and 4.3) that Inf-Sup stability does not hold. More precisely, we prove that $\beta_{\mathcal{T}}=0$ in the uniform conforming case, and that $\beta_{\mathcal{T}}>0$ with

$$
\beta_{\mathcal{T}} \underset{\operatorname{size}(\mathcal{T}) \rightarrow 0}{\longrightarrow} 0
$$

in the non-conforming case. 


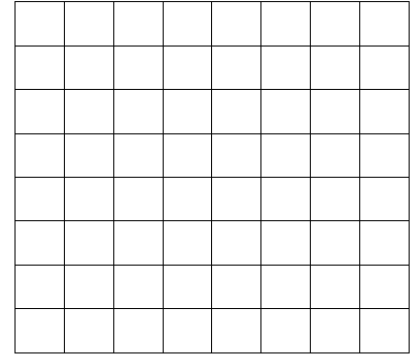

(a) Uniform conforming mesh

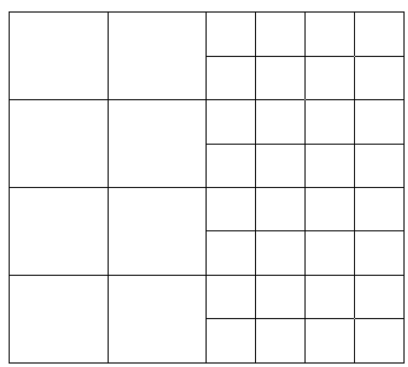

(b) Non-conforming mesh

Figure 7. The Cartesian meshes under study

This behavior of $\beta_{\mathcal{T}}$ proves the existence of at least one unstable pressure mode. More precisely, we prove (Theorem 4.4) that there is in fact only one such unstable mode. It means that, the Inf-Sup stability property holds if we impose the pressure fields to be orthogonal to the unstable mode, or if we add a suitable rank one stabilisation term in the divergence equation. We call this property the codimension 1 Inf-Sup stability.

Actually, this is consistent with the fact that, in practice, the DDFV scheme behaves very well on such kinds of meshes. To illustrate this fact, we show in Figure 8 , the $L^{2}$-error for the velocity and the pressure using the non-conforming grid of Figure $7 \mathrm{~b}$ for the smooth exact solution given by

$$
u(x, y)=\left(\begin{array}{c}
-2 \pi \sin ^{2}(\pi x) \cos (\pi y) \sin (\pi y) \\
2 \pi \sin ^{2}(\pi y) \cos (\pi x) \sin (\pi x)
\end{array}\right) \text { and } p(x, y)=x+y-1 .
$$

We observe the second order convergence for both velocity and pressure.

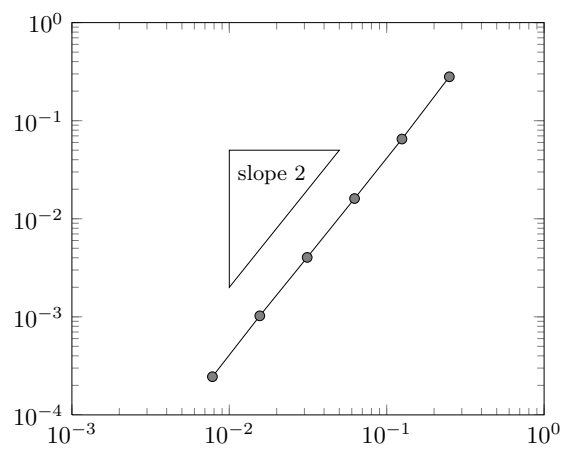

(a) $L^{2}$-Error for the velocity

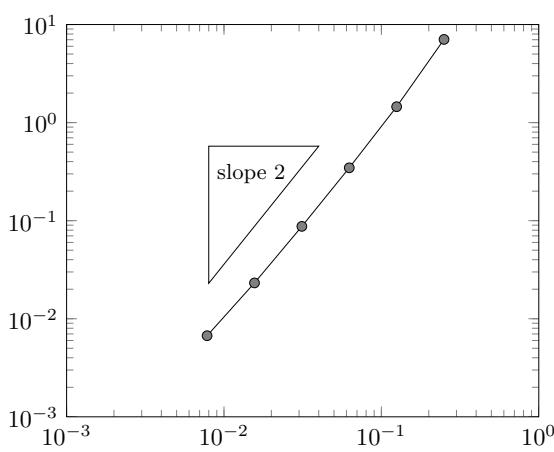

(b) $L^{2}$-Error for the pressure

Figure 8. $L^{2}$-error as a function of the mesh size for nonconforming mesh (see Figure $7 \mathrm{~b}$ ) 
4.1. Inf-Sup instability. The meshes we consider in this section are Cartesian. This means that all primal edges are either horizontal, either vertical (note that this does not necessarily hold for dual edges). Therefore, we can adopt the following notations:

- $\mathfrak{D}^{h}$ is the set of diamond cells whose associated primal edge is horizontal,

- $\mathfrak{D}^{v}$ is the set of diamond cells whose associated primal edge is vertical.

Similarly, we denote by $\partial \Omega^{h}$ (resp. $\partial \Omega^{v}$ ) the horizontal (resp. vertical) part of the boundary of the domain.

We will prove in the sequel that the unstable part of the scheme is completely contained in a pressure mode which looks like a checkerboard defined as follows.

Definition 4.1 (Checkerboard mode). The checkerboard mode $\psi^{\mathfrak{D}}$ is defined by:

$$
\psi^{\mathcal{D}}= \begin{cases}+1, & \text { for } \mathcal{D} \in \mathfrak{D}^{v}, \\ -1, & \text { for } \mathcal{D} \in \mathfrak{D}^{h} .\end{cases}
$$

Observe that $m\left(\psi^{\mathfrak{D}}\right)=0$ and $\left\|\psi^{\mathfrak{D}}\right\|_{\mathfrak{D}, 2}=1$ for the two kinds of Cartesian meshes studied in this section (see Figure 7 ).

We easily obtain the following result.

Theorem 4.2 (Inf-Sup instability for uniform Cartesian mesh). For a uniform Cartesian mesh $\mathcal{T}$, the checkerboard mode $\psi^{\mathfrak{D}}$ satisfies

$$
b_{\mathcal{T}}\left(\mathbf{v}^{\mathcal{T}}, \psi^{\mathfrak{D}}\right)=0, \quad \forall \mathbf{v}^{\mathcal{T}} \in \mathbb{E}_{0} .
$$

As a consequence, we have $\beta_{\mathcal{T}}=0$.

Proof. By definition of $\psi^{\mathfrak{D}}$, and since all primal and interior dual cells are squares, we have

$$
\nabla^{\mathcal{K}} \psi^{\mathfrak{D}}=0, \quad \forall \mathcal{K} \in \mathfrak{M}, \quad \text { and } \quad \nabla^{\mathcal{K}^{*}} \psi^{\mathfrak{D}}=0, \quad \forall \mathcal{K}^{*} \in \mathfrak{M}^{*} .
$$

This obviously implies that

$$
b_{\mathcal{T}}\left(\mathbf{v}^{\mathcal{T}}, \psi^{\mathfrak{D}}\right)=-\llbracket \mathbf{v}^{\mathcal{T}}, \nabla^{\mathcal{T}} \psi^{\mathfrak{D}} \rrbracket_{\mathcal{T}}=0 .
$$

In the case of the non-conforming Cartesian meshes, the analysis is not so easy and we will prove that $\nabla^{\mathcal{T}} \psi^{\mathcal{D}}$ does not vanish but is "small" in a suitable sense. This implies that $\beta_{\mathcal{T}}$ is not zero in that case, but tends to 0 as $\operatorname{size}(\mathcal{T}) \rightarrow 0$. The precise result is the following.

Theorem 4.3 (Inf-Sup instability for non-conforming Cartesian mesh). For the meshes shown in Figure $7 \mathrm{~b}$, there exist $C_{5}, C_{6}>0$ which do not depend on $\operatorname{size}(\mathcal{T})$, such that the checkerboard mode $\psi^{\mathfrak{D}}$ satisfies

$$
C_{5} \operatorname{size}(\mathcal{T})^{\frac{1}{2}} \leq \sup _{\mathbf{v} \mathcal{T} \in \mathbb{E}_{0}} \frac{b_{\mathcal{T}}\left(\mathbf{v}^{\mathcal{T}}, \psi^{\mathfrak{D}}\right)}{\left\|\nabla^{\mathfrak{D}} \mathbf{v}^{\mathcal{T}}\right\|_{\mathfrak{D}, 2}} \leq C_{6} \operatorname{size}(\mathcal{T})^{\frac{1}{2}}
$$

This implies in particular that $\beta_{\mathcal{T}} \leq C_{6} \operatorname{size}(\mathcal{T})^{\frac{1}{2}}$.

Proof. The proof is divided into different steps. For any $\mathbf{v}^{\mathcal{T}} \in \mathbb{E}_{0}$, we first evaluate the contribution of the primal cells in $b_{\mathcal{T}}\left(\mathbf{v}^{\mathcal{T}}, \psi^{\mathfrak{D}}\right)$, then one of the dual cells in order to get (4.1). The Definition 4.1 of the checkerboard mode $\psi^{\mathfrak{D}}$ then leads to the upper bound. To conclude, with a particular choice of the discrete velocity $\mathbf{v}^{\mathcal{T}}$, we are able to prove the lower bound.

To simplify the notations in this proof, we denote by $h$ the length of the primal edges of the coarse part of the mesh. 
- We first show that, for any $\mathbf{v}^{\mathcal{T}} \in \mathbb{E}_{0}$, the primal control volumes do not contribute to $b_{\mathcal{T}}\left(\mathbf{v}^{\mathcal{T}}, \psi^{\mathfrak{D}}\right)$.

We can notice that if $\mathcal{K} \in \mathfrak{M}$ has exactly four primal edges, then the same computation as the one for uniform Cartesian meshes shows that $\nabla^{\mathcal{K}} \psi^{\mathfrak{D}}=0$. It remains to study the case of primal control volumes with five edges, that is the ones situated just on the left-side of the interface (see Figure 9). For such a primal cell $\mathcal{K}_{i}^{l}$, we have

$$
m_{\mathcal{K}_{i}^{l}} \nabla^{\mathcal{K}_{i}^{l}} \psi^{\mathcal{D}}=h\left(\psi^{\mathcal{D}_{i+1 / 2}^{l}}-\psi^{\mathcal{D}_{i-1 / 2}^{l}}\right) \mathbf{e}_{\boldsymbol{y}}+\frac{h}{2}\left(\psi^{\mathcal{D}_{i}^{+}}+\psi^{\mathcal{D}_{i}^{-}}-2 \psi^{\mathcal{D}_{i}^{l}}\right) \mathbf{e}_{\boldsymbol{x}}
$$

Since $\mathcal{D}_{i}^{+}, \mathcal{D}_{i}^{-}, \mathcal{D}_{i}^{l}$ are vertical diamond cells and $\mathcal{D}_{i+1 / 2}^{l}, \mathcal{D}_{i-1 / 2}^{l}$ are horizontal diamond cells, we have

$$
\psi^{\mathcal{D}_{i}^{+}}=\psi^{\mathcal{D}_{i}^{-}}=\psi^{\mathcal{D}_{i}^{l}}=1 \text { and } \psi^{\mathcal{D}_{i+1 / 2}^{l}}=\psi^{\mathcal{D}_{i-1 / 2}^{l}}=-1,
$$

so that we also have in that case $\nabla^{\kappa_{i}^{l}} \psi^{\mathfrak{D}}=0$.

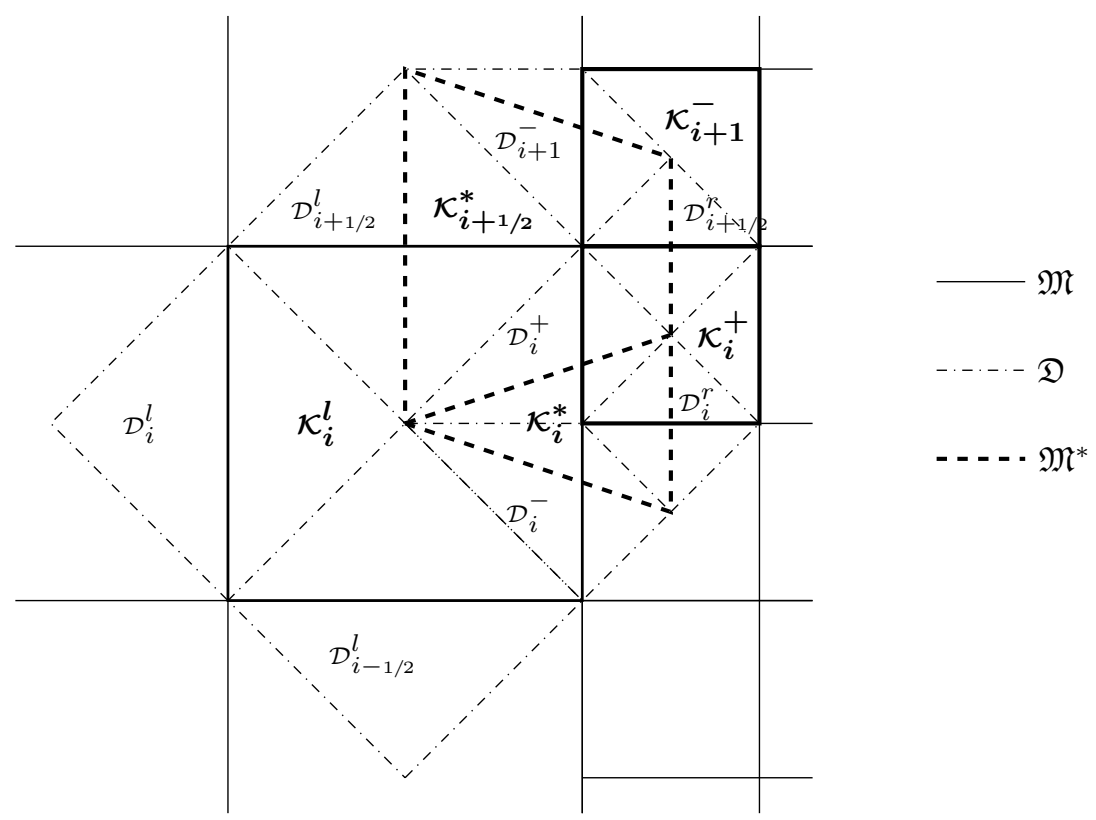

Figure 9. Some notations near the interface for the nonconforming Cartesian mesh, Figure $7 \mathrm{~b}$

- Let us now consider dual control volumes. For a $\mathcal{K}^{*} \in \mathfrak{M}^{*}$ whose associated vertex $x_{\mathcal{K}^{*}}$ is not on the interface $I$, we have $\nabla^{\mathcal{K}^{*}} \psi^{\mathfrak{D}}=0$. This is the same proof as for uniform Cartesian meshes.

It remains to study the contributions of dual cells associated with vertices located on the interface. The geometry of the mesh is such that, there are two kinds of such dual cells. Some of them are triangle cells $\mathcal{K}_{i}^{*}$, for $i=1, \ldots, N$, the others are trapeze cells $\mathcal{K}_{i+1 / 2}^{*}$, for $i=1, \ldots, N-1$ (see Figure 91). Here, we have set $N=n_{e} / 2, n_{e}$ being the total number of primal edges which constitute the interface. 
- A straightforward computation shows that in triangle dual cells $\mathcal{K}_{i}^{*}$ we have

$$
\begin{aligned}
m_{\mathcal{K}_{i}^{*}} \nabla^{\mathcal{K}_{i}^{*}} \psi^{\mathfrak{D}} & =\frac{h}{2}\left(\psi^{\mathcal{D}_{i}^{r}}-\frac{1}{2}\left(\psi^{\mathcal{D}_{i}^{-}}+\psi^{\mathcal{D}_{i}^{+}}\right)\right) \mathbf{e}_{\boldsymbol{x}}+\frac{3 h}{4}\left(\psi^{\mathcal{D}_{i}^{+}}-\psi^{\mathcal{D}_{i}^{-}}\right) \mathbf{e}_{\boldsymbol{y}} \\
& =-h \mathbf{e}_{\boldsymbol{x}}, \quad \forall i=1, \ldots, N
\end{aligned}
$$

- For trapeze dual cells $\mathcal{K}_{i+1 / 2}^{*}$, we immediately get

$$
\begin{aligned}
m_{\mathcal{K}_{i+1 / 2}^{*}} \nabla^{\mathcal{K}_{i+1 / 2}^{*}} \psi^{\mathfrak{D}}= & h\left(\frac{1}{2} \psi^{\mathcal{D}_{i+1 / 2}^{r}}-\psi^{\mathcal{D}_{i+1 / 2}^{l}}+\frac{1}{4}\left(\psi^{\mathcal{D}_{i}^{+}}+\psi^{\mathcal{D}_{i+1}^{-}}\right)\right) \mathbf{e}_{\boldsymbol{x}} \\
& +\frac{3 h}{4}\left(\psi^{\mathcal{D}_{i+1}^{-}}-\psi^{\mathcal{D}_{i}^{+}}\right) \mathbf{e}_{\boldsymbol{y}} \\
= & h \mathbf{e}_{\boldsymbol{x}}, \quad \forall i=1, \ldots, N-1 .
\end{aligned}
$$

We observe that the orientation of the two gradients are exactly opposed; this is precisely the reason why we are able to prove that the contribution of these terms in $b_{\mathcal{T}}\left(\mathbf{v}^{\mathcal{T}}, \psi^{\mathfrak{D}}\right)$ is small. More precisely, for any $\mathbf{v}^{\mathcal{T}} \in \mathbb{E}_{0}$, we have

$$
b_{\mathcal{T}}\left(\mathbf{v}^{\mathcal{T}}, \psi^{\mathfrak{D}}\right)=-\frac{h}{2} \sum_{i=1}^{N}\left(\mathbf{v}_{\mathcal{K}_{i+1 / 2}^{*}}-\mathbf{v}_{\mathcal{K}_{i}^{*}}\right) \cdot \mathbf{e}_{\boldsymbol{x}}
$$

where, we recall that, the boundary value $\mathbf{v}_{\mathcal{K}_{N+1 / 2}^{*}}$ is equal to 0 since $\mathbf{v}^{\mathcal{T}} \in$ $\mathbb{E}_{0}$.

- For any $1 \leq i \leq N$, we consider the diamond cell $\mathcal{D}_{i}^{+}$. By definition of the velocity discrete gradient on $\mathcal{D}_{i}^{+}$, we have

$$
\left(\mathbf{v}_{\mathcal{K}_{i+1 / 2}^{*}}-\mathbf{v}_{\mathcal{K}_{i}^{*}}\right) \cdot \mathbf{e}_{\boldsymbol{x}}=\frac{h}{2}{ } \mathbf{e}_{\boldsymbol{x}} \cdot \nabla^{\mathcal{D}_{i}^{+}} \mathbf{v}^{\mathcal{T}} \cdot \mathbf{e}_{\boldsymbol{y}}
$$

Therefore, for some $C>0$ independent of the mesh size, we get

$$
\frac{h}{2}\left|\left(\mathbf{v}_{\mathcal{K}_{i+1 / 2}^{*}}-\mathbf{v}_{\mathcal{K}_{i}^{*}}\right) \cdot \mathbf{e}_{\boldsymbol{x}}\right| \leq C m_{\mathcal{D}_{i}^{+}}\left|\nabla^{\mathcal{D}_{i}^{+}} \mathbf{v}^{\mathcal{T}}\right| .
$$

It follows, by the Cauchy-Schwarz inequality that

$$
\begin{aligned}
b\left(\mathbf{v}^{\mathcal{T}}, \psi^{\mathfrak{D}}\right) & \leq C \sum_{i=1}^{N} m_{\mathcal{D}_{i}^{+}}\left|\nabla^{\mathcal{D}_{i}^{+}} \mathbf{v}^{\mathcal{T}}\right| \leq C\left\|\nabla^{\mathfrak{D}} \mathbf{v}^{\mathcal{T}}\right\|_{\mathfrak{D}, 2}\left(\sum_{i=1}^{N} m_{\mathcal{D}_{i}^{+}}\right)^{\frac{1}{2}} \\
& \leq C\left\|\nabla^{\mathfrak{D}} \mathbf{v}^{\mathcal{T}}\right\|_{\mathfrak{D}, 2} \operatorname{size}(\mathcal{T})^{\frac{1}{2}},
\end{aligned}
$$

and the upper bound is proved.

- It remains to prove the lower bound. To this end, we build a particular discrete velocity field $\mathbf{v}^{\mathcal{T}} \in \mathbb{E}_{0}$ parallel to $\mathbf{e}_{\boldsymbol{x}}$, which is zero everywhere except for its horizontal component on each triangle dual cell on the interface $\mathcal{K}_{i}^{*}$, $i=1, \ldots, N$, for which we choose $\mathbf{v}_{\mathcal{K}_{i}^{*}}=\mathbf{e}_{\boldsymbol{x}}$.

From (4.1) and straightforward computations we get

$$
b\left(\mathbf{v}^{\mathcal{T}}, \psi^{\mathfrak{D}}\right)=\frac{h N}{2}=\frac{1}{2} \quad \text { and } \quad\left\|\nabla^{\mathfrak{D}} \mathbf{v}^{\mathcal{T}}\right\|_{\mathfrak{D}, 2} \leq \frac{C}{\operatorname{size}(\mathcal{T})^{\frac{1}{2}}},
$$

which imply the required lower bound. 
4.2. Inf-Sup stability up to the checkerboard mode. Despite the Inf-Sup instability of the DDFV scheme on Cartesian meshes that we established in the previous section, it is observed that the scheme is very accurate (see Figure 8). Note that, in the case of uniform Cartesian meshes, it is needed to add some stabilization term (if not, the scheme is not well-posed since $\beta_{\mathcal{T}}=0$ ), but the magnitude of this stabilization term does not seem to have any influence on the accuracy of the method.

We propose an interpretation of this surprisingly good behavior by proving that there is essentially one single unstable mode and that, in the orthogonal of this mode, the uniform Inf-Sup inequality holds. Moreover, this unstable mode is close to (but not always equal to) the checkerboard mode $\psi^{\mathfrak{D}}$ that we identified before (see Theorem 4.77). We refer for instance to the discussion in [9, Section II.3].

We first illustrate numerically this phenomenon in Figure 10, by plotting as a function of $\operatorname{size}(\mathcal{T})$ :

- the value of $\beta_{\mathcal{T}}$,

- the value of $\sqrt{\lambda_{3}\left(S_{\mathcal{T}}\right)}$ which is the next eigenvalue of the Schur complement of the system,

- the value of the following co-dimension 1 Inf-Sup constant

$$
\widetilde{\beta}_{\mathcal{T}}=\inf _{\substack{p^{\mathfrak{D}} \in\left\{\psi^{\mathfrak{D}}\right\}^{\perp} \\ m\left(p^{\mathfrak{D}}\right)=0}}\left(\sup _{\mathbf{v}^{\mathcal{T}} \in \mathbb{E}_{0}} \frac{b_{\mathcal{T}}\left(\mathbf{v}^{\mathcal{T}}, p^{\mathfrak{D}}\right)}{\left\|\nabla^{\mathfrak{D}} \mathbf{v}^{\mathcal{T}}\right\|_{\mathfrak{D}, 2}\left\|p^{\mathfrak{D}}\right\|_{\mathfrak{D}, 2}}\right)
$$

where $\left\{\psi^{\mathfrak{D}}\right\}^{\perp}=\left\{p^{\mathfrak{D}} \in \mathbb{R}^{\mathfrak{D}}:\left\langle M_{\mathcal{T}} p^{\mathfrak{D}}, \psi^{\mathfrak{D}}\right\rangle=0\right\}$. Note that $\widetilde{\beta}_{\mathcal{T}}$ can also be computed by solving a suitable modified eigenvalue problem for which we do not give the details here.

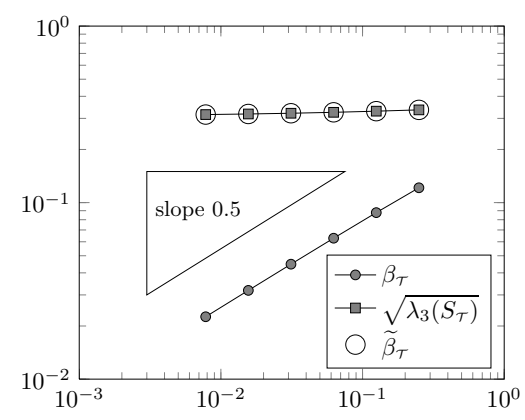

(a) Comparison between $\beta_{\mathcal{T}}, \sqrt{\lambda_{3}\left(S_{\mathcal{T}}\right)}$ and $\widetilde{\beta_{\mathcal{T}}}$

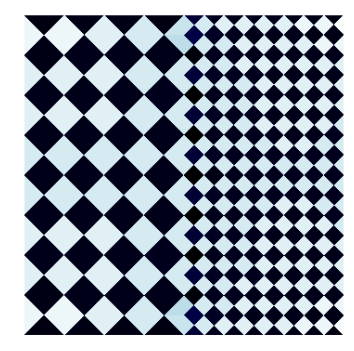

(b) The unstable mode

Figure 10. Inf-Sup instability for the non-conforming Cartesian mesh, see Figure $7 \mathrm{~b}$

We observe on Figure 10a that, in accordance with Theorem 4.3 , we have $\beta_{\mathcal{T}} \rightarrow 0$ when $\operatorname{size}(\mathcal{T}) \rightarrow 0$. We also observe that $\sqrt{\lambda_{3}\left(S_{\mathcal{T}}\right)}$ is bounded from below, which confirms the presence of a single unstable mode. Moreover, $\widetilde{\beta}_{\mathcal{T}}$ also appears to be bounded from below and seems to almost coincide with $\sqrt{\lambda_{3}\left(S_{\mathcal{T}}\right)}$. This suggests that the unstable mode (that is the pressure mode for which the Inf-Sup inequality 
is an equality, which is related to the eigenvector of $S_{\mathcal{T}}$ associated with $\lambda_{2}\left(S_{\mathcal{T}}\right)$ ) should be not too far from the checkerboard mode $\psi^{\mathfrak{D}}$ that we introduced below. This is confirmed in Figure 10b where we plot the computed unstable mode, refered to as $q^{\mathfrak{D}}$.

Even if we do not have an explicit formula for this mode, we will prove in Theorem 4.7 that $\left\|q^{\mathfrak{D}}-\psi^{\mathfrak{D}}\right\|_{\mathfrak{D}, 2} \leq C \operatorname{size}(\mathcal{T})^{\frac{1}{2}}$.

We are now in position to provide a theoretical justification of these observations in the following result.

Theorem 4.4 (Codimension 1 Inf-Sup stability). Let be $\mathcal{T}$ a uniform or nonconforming Cartesian DDFV mesh as described in Figure 7 . There exists a $C_{7}>0$ which does not depend on $\operatorname{size}(\mathcal{T})$ such that for any $p^{\mathfrak{D}} \in \mathbb{R}^{\mathfrak{D}}$ we have

$$
\left[\left(p^{\mathfrak{D}}, \psi^{\mathfrak{D}}\right)_{\mathfrak{D}}=0 \text { and } m\left(p^{\mathfrak{D}}\right)=0\right] \Longrightarrow\left[C_{7}\left\|p^{\mathfrak{D}}\right\|_{\mathfrak{D}, 2} \leq \sup _{\mathbf{v} \mathcal{T} \in \mathbb{E}_{0}} \frac{b_{\mathcal{T}}\left(\mathbf{v}^{\mathcal{T}}, p^{\mathfrak{D}}\right)}{\left\|\nabla^{\mathfrak{D}} \mathbf{v}^{\mathcal{T}}\right\|_{\mathfrak{D}, 2}}\right]
$$

which means that $\widetilde{\beta}_{\mathcal{T}} \geq C_{7}$.

This theorem relies on the following proposition which is a suitable generalisation of Proposition 3.1 adapted to the framework under study.

Proposition 4.5. Let be $\mathcal{T}$ a uniform or non-conforming Cartesian DDFV mesh as described in Figure 7 . There exists a $\alpha_{\mathcal{T}}>0$, independent of $\operatorname{size}(\mathcal{T})$, such that for any $\mathbf{v}^{h}, \mathbf{v}^{v} \in\left(H_{0}^{1}(\Omega)\right)^{2}$ satisfying

$$
\operatorname{div} \mathbf{v}^{h}=0 \text { on } \bigcup_{\mathcal{D} \in \mathfrak{D}^{v}} \mathcal{D}, \quad \text { and } \quad \operatorname{div} \mathbf{v}^{v}=0 \text { on } \bigcup_{\mathcal{D} \in \mathfrak{D}^{h}} \mathcal{D},
$$

there exists $a \mathbf{v}^{\mathcal{T}} \in \mathbb{E}_{0}$ such that

$$
\left\|\nabla^{\mathfrak{D}} \mathbf{v}^{\mathcal{T}}\right\|_{\mathfrak{D}, 2} \leq \alpha_{\mathcal{T}}\left(\left\|\mathbf{v}^{h}\right\|_{H^{1}}+\left\|\mathbf{v}^{v}\right\|_{H^{1}}\right)
$$

and, for any $p^{\mathfrak{D}} \in \mathbb{R}^{\mathfrak{D}}$,

$$
\left|\sum_{\mathcal{D} \in \mathfrak{D}} \int_{\mathcal{D}} p^{\mathcal{D}}\left(\operatorname{div}^{\mathcal{D}}\left(\mathbf{v}^{\mathcal{T}}\right)-\operatorname{div}\left(\frac{\mathbf{v}^{h}+\mathbf{v}^{v}}{2}\right)\right)\right| \leq \alpha_{\mathcal{T}}\left(\left\|\mathbf{v}^{h}\right\|_{H^{1}}+\left\|\mathbf{v}^{v}\right\|_{H^{1}}\right)\left\|h^{\mathcal{T}} \nabla^{\mathcal{T}} p^{\mathfrak{D}}\right\|_{\mathcal{T}, 2} .
$$

Let us first give the proof of Theorem 4.4 using this proposition.

Proof. Let be $p^{\mathfrak{D}} \in \mathbb{R}^{\mathfrak{D}}$ such that $m\left(p^{\mathfrak{D}}\right)=0$ and $\left(p^{\mathfrak{D}}, \psi^{\mathfrak{D}}\right)_{\mathfrak{D}}=0$. We define the "vertical" and "horizontal" parts of this pressure field defined by

$$
p^{\mathfrak{D}^{v}}=\sum_{\mathcal{D} \in \mathfrak{D}^{v}} p^{\mathcal{D}} 1_{\mathcal{D}}, \text { and } p^{\mathfrak{D}^{h}}=\sum_{\mathcal{D} \in \mathfrak{D}^{h}} p^{\mathcal{D}} 1_{\mathcal{D}}
$$

where $1_{\mathcal{D}}$ is the indicator function of $\mathcal{D}$. By definition of the checkerboard mode $\psi^{\mathfrak{D}}$ (see Definition 4.1) we observe that

$$
\int_{\Omega} p^{\mathfrak{D}^{v}}+\int_{\Omega} p^{\mathfrak{D}^{h}}=m\left(p^{\mathfrak{D}}\right)=0 \text { and } \int_{\Omega} p^{\mathfrak{D}^{v}}-\int_{\Omega} p^{\mathfrak{D}^{h}}=\left(p^{\mathfrak{D}}, \psi^{\mathfrak{D}}\right)_{\mathfrak{D}}=0 .
$$

It follows that both $p^{\mathfrak{D}^{h}}$ and $p^{\mathfrak{D}^{v}}$ have a zero mean-value so that we can apply Proposition 1.1 and set $\mathbf{v}^{h}=\Pi\left(p^{\mathfrak{D}^{h}}\right), \mathbf{v}^{v}=\Pi\left(p^{\mathfrak{D}^{v}}\right)$.

We can see that, by construction, $\operatorname{div} \mathbf{v}^{h}=0$ on all the vertical diamond cells, $\operatorname{div} \mathbf{v}^{v}=0$ on all the horizontal diamond cells, and that $\operatorname{div} \mathbf{v}^{h}+\operatorname{div} \mathbf{v}^{v}=p^{\mathfrak{D}}$. We now take $\mathbf{v}^{\mathcal{T}} \in \mathbb{E}_{0}$ given by Proposition 4.5 which satisfies

$$
\left\|\nabla^{\mathfrak{D}} \mathbf{v}^{\mathcal{T}}\right\|_{\mathfrak{D}, 2} \leq \alpha_{\mathcal{T}}\left(\left\|\mathbf{v}^{h}\right\|_{H^{1}}+\left\|\mathbf{v}^{v}\right\|_{H^{1}}\right) \leq 2 \alpha_{\mathcal{T}}\|\Pi\|\left\|p^{\mathfrak{D}}\right\|_{\mathfrak{D}, 2} .
$$


We can now compute

$$
b_{\mathcal{T}}\left(\mathbf{v}^{\mathcal{T}}, p^{\mathfrak{D}}\right)=\frac{1}{2}\left\|p^{\mathfrak{D}}\right\|_{\mathfrak{D}, 2}^{2}+\sum_{\mathcal{D} \in \mathfrak{D}} \int_{\mathcal{D}} p^{\mathcal{D}}\left(\operatorname{div}^{\mathcal{D}}\left(\mathbf{v}^{\mathcal{T}}\right)-\operatorname{div}\left(\frac{\mathbf{v}^{h}+\mathbf{v}^{v}}{2}\right)\right) .
$$

According to Proposition 4.5 we deduce

$$
b_{\mathcal{T}}\left(\mathbf{v}^{\mathcal{T}}, p^{\mathfrak{D}}\right) \geq \frac{1}{2}\left\|p^{\mathfrak{D}}\right\|_{\mathfrak{D}, 2}^{2}-2 \alpha_{\mathcal{T}}\|\Pi\|\left\|p^{\mathfrak{D}}\right\|_{\mathfrak{D}, 2}\left\|h^{\mathcal{T}} \nabla^{\mathcal{T}} p^{\mathfrak{D}}\right\|_{\mathcal{T}, 2} .
$$

- In the case where $\left\|h^{\mathcal{T}} \nabla^{\mathcal{T}} p^{\mathfrak{D}}\right\|_{\mathcal{T}, 2} \leq \frac{1}{8 \alpha \mathcal{\mathcal { T }}\|\Pi\|}\left\|p^{\mathfrak{D}}\right\|_{\mathfrak{D}, 2}$, the estimate (4.6) directly gives

$$
\frac{b_{\mathcal{T}}\left(\mathbf{v}^{\mathcal{T}}, p^{\mathfrak{D}}\right)}{\left\|\nabla^{\mathfrak{D}} \mathbf{v}^{\mathcal{T}}\right\|_{\mathfrak{D}, 2}} \geq \frac{1}{4} \frac{\left\|p^{\mathfrak{D}}\right\|_{\mathfrak{D}, 2}^{2}}{\left\|\nabla^{\mathfrak{D}} \mathbf{v}^{\mathcal{T}}\right\|_{\mathfrak{D}, 2}} \geq \frac{1}{8 \alpha_{\mathcal{T}}\|\Pi\|}\left\|p^{\mathfrak{D}}\right\|_{\mathfrak{D}, 2},
$$

and the claim is proved.

- Assume now that $\left\|h^{\mathcal{T}} \nabla^{\mathcal{T}} p^{\mathfrak{D}}\right\|_{\mathcal{T}, 2} \geq \frac{1}{8 \alpha_{\mathcal{T}}\|\Pi\|}\left\|p^{\mathfrak{D}}\right\|_{\mathfrak{D}, 2}$. In that case, defining $\mathbf{v}^{\mathcal{T}}$ by (3.9) as in Theorem 3.2 gives the claim in the same way.

It remains to give a proof of the preliminary proposition. To this end, we use the following approximation lemma which is a straightforward consequence of usual results in the finite volume framework (see for instance [2, Lemma 3.3]).

Lemma 4.6. There exists a $C>0$, such that if $\sigma_{1}$ and $\sigma_{2}$ are two segments in $\mathbb{R}^{2}$ contained in a bounded convex set $\mathcal{P}$ with non-empty interior, we have

$$
\left|\frac{1}{m_{\sigma_{1}}} \int_{\sigma_{1}} \mathbf{v}-\frac{1}{m_{\sigma_{2}}} \int_{\sigma_{2}} \mathbf{v}\right| \leq C\left(\frac{1}{m_{\sigma_{1}}}+\frac{1}{m_{\sigma_{2}}}\right) \frac{\operatorname{diam}(\mathcal{P})^{2}}{m_{\mathcal{P}}} \int_{\mathcal{P}}|\nabla \mathbf{v}|,
$$

for any $\mathbf{v} \in W^{1,1}\left(\mathbb{R}^{2}\right)$.

Proof of Proposition 4.5. In order to simplify the presentation of the proof, we set $\mathbf{v}^{v, h} \stackrel{\text { def }}{=} \frac{\mathbf{v}^{v}+\mathbf{v}^{h}}{2}$.

The proof is divided into different steps. We first deal with the conforming case (which is simpler) and then with the non-conforming one for which specific care is needed for the control volumes near the interface.

We will first start by giving an explicit formula (4.8) for the discrete velocity $\mathbf{v}^{\mathcal{T}} \in \mathbb{E}_{0}$ that will fulfill all the requirements. The stability estimate (4.4) will then be a simple consequence of Lemma 4.6.

The difficult part will be to prove (4.5). In the case of uniform meshes, we will obtain that only the boundary diamond cells actually contribute to the estimate and that the sum of all the contributions can be bounded by some quantity depending on the velocity gradient and the discrete pressure gradients. For non-conforming meshes, additional contributions coming from diamond cells near the interface have to be taken carefully into account.

- For any $\mathcal{K} \in \mathfrak{M}$ (resp. $\mathcal{K}^{*} \in \mathfrak{M}^{*}$ ), we consider two segments $\gamma_{\mathcal{K}}^{v}$ and $\gamma_{\mathcal{K}}^{h}$ (resp. $\gamma_{\mathcal{K}^{*}}^{v}$ and $\left.\gamma_{\mathcal{K}^{*}}^{h}\right)$ in $\Omega$ passing through the point $x_{\mathcal{K}}$ (resp. $x_{\mathcal{K}^{*}}$ ) as described in Figure [1]. Notice that $\gamma_{\mathcal{K}^{*}}^{v}$ is horizontal and $\gamma_{\mathcal{K}^{*}}^{h}$ is vertical; this is due to the fact that the superscript indicates the kind of diamond (horizontal or vertical) for which each $\gamma_{\bullet}^{\bullet}$ will contribute and not its orientation. 


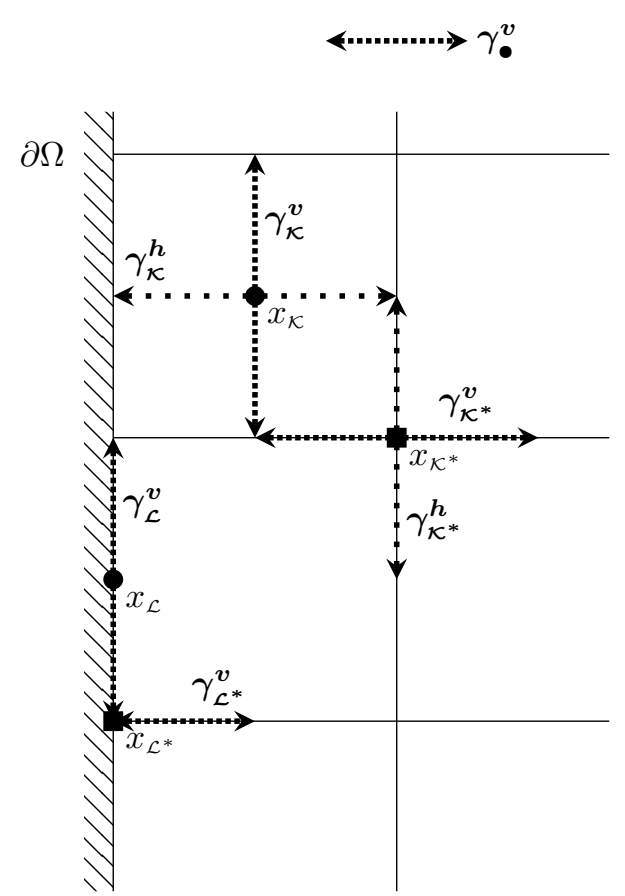

(a) Conforming mesh

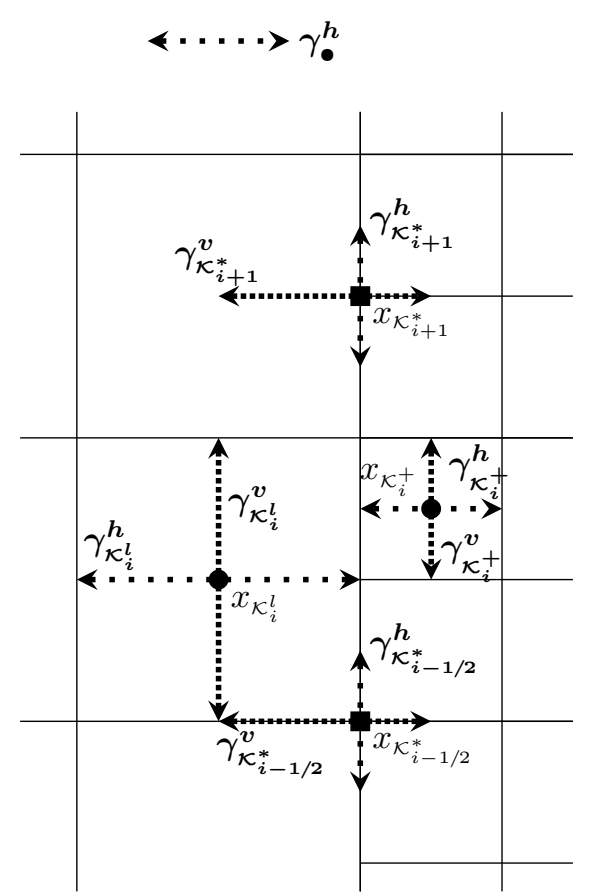

(b) Non-conforming mesh

Figure 11. Definition of the segments $\gamma_{\bullet}^{\bullet}$

We can now build $\mathbf{v}^{\mathcal{T}} \in \mathbb{E}_{0}$ by setting

$$
\begin{cases}\mathbf{v}_{\mathcal{K}} \cdot \mathbf{e}_{\boldsymbol{x}}=\frac{1}{m_{\gamma_{\mathcal{K}}^{v}}} \int_{\gamma_{\mathcal{K}}^{v}} \mathbf{v}^{v} \cdot \mathbf{e}_{\boldsymbol{x}}, & \mathbf{v}_{\mathcal{K}} \cdot \mathbf{e}_{\boldsymbol{y}}=\frac{1}{m_{\gamma_{\mathcal{K}}^{h}}} \int_{\gamma_{\mathcal{K}}^{h}} \mathbf{v}^{h} \cdot \mathbf{e}_{\boldsymbol{y}}, \quad \forall \mathcal{K} \in \mathfrak{M}, \\ \mathbf{v}_{\mathcal{K}^{*}} \cdot \mathbf{e}_{\boldsymbol{x}}=\frac{1}{m_{\gamma_{\mathcal{K}^{*}}^{h}}} \int_{\gamma_{\mathcal{K}^{*}}^{h}} \mathbf{v}^{h} \cdot \mathbf{e}_{\boldsymbol{x}}, & \mathbf{v}_{\mathcal{K}^{*}} \cdot \mathbf{e}_{\boldsymbol{y}}=\frac{1}{m_{\gamma_{\mathcal{K}^{*}}^{v}}} \int_{\gamma_{\mathcal{K}^{*}}^{v}} \mathbf{v}^{v} \cdot \mathbf{e}_{\boldsymbol{y}}, \quad \forall \mathcal{K}^{*} \in \mathfrak{M}^{*} .\end{cases}
$$

- Let us prove the stability estimate (4.4). We observe that

$$
m_{\mathcal{D}}\left|\nabla^{\mathcal{D}} \mathbf{v}^{\mathcal{T}}\right|^{2} \leq C(\operatorname{reg}(\mathcal{T}))\left(\left|\mathbf{v}_{\mathcal{K}}-\mathbf{v}_{\mathcal{L}}\right|^{2}+\left|\mathbf{v}_{\mathcal{K}^{*}}-\mathbf{v}_{\mathcal{L}^{*}}\right|^{2}\right),
$$

and, with Lemma 4.6.

$$
\begin{gathered}
\left|\mathbf{v}_{\mathcal{K}}-\mathbf{v}_{\mathcal{L}}\right|^{2} \leq C(\operatorname{reg}(\mathcal{T})) \int_{\widehat{\mathcal{K} \cup \mathcal{L}}}\left(\left|\nabla \mathbf{v}^{v}\right|^{2}+\left|\nabla \mathbf{v}^{h}\right|^{2}\right), \\
\left|\mathbf{v}_{\mathcal{K}^{*}}-\mathbf{v}_{\mathcal{L}^{*}}\right|^{2} \leq C(\operatorname{reg}(\mathcal{T})) \int_{\widehat{\mathcal{K}^{*} \cup \mathcal{L}^{*}}}\left(\left|\nabla \mathbf{v}^{v}\right|^{2}+\left|\nabla \mathbf{v}^{h}\right|^{2}\right),
\end{gathered}
$$

Using that the set of all the convex hulls of the kind $\widehat{\mathcal{U} \cup \mathcal{L}}$, for instance, covers the domain $\Omega$ at most 5 times, we can sum all these inequalities in order to finally get

$$
\left\|\nabla^{\mathfrak{D}} \mathbf{v}^{\mathcal{T}}\right\|_{\mathfrak{D}, 2}^{2}=\sum_{\mathcal{D} \in \mathfrak{D}} m_{\mathcal{D}}\left|\nabla^{\mathcal{D}} \mathbf{v}^{\mathcal{T}}\right|^{2} \leq C(\operatorname{reg}(\mathcal{T}))\left(\left\|\mathbf{v}^{v}\right\|_{H^{1}}^{2}+\left\|\mathbf{v}^{h}\right\|_{H^{1}}^{2}\right)
$$

- Let us prove (4.5) in the case of a uniform Cartesian mesh. Let $p^{\mathfrak{D}} \in \mathbb{R}^{\mathfrak{D}}$ and $\mathcal{D}$ be any diamond cell. We need to consider different cases. 
- The case where $\mathcal{D}$ is not a boundary diamond cell. We assume for instance that $\mathcal{D} \in \mathfrak{D}^{v} \backslash \mathfrak{D}_{\text {ext }}$, the case of a horizontal diamond cell being similar. We refer to Figure 11a for the notations.

Using the definition of the discrete divergence operator $\operatorname{div}^{\mathfrak{D}}$ (see Definition 2.2) and the one of $\mathbf{v}^{\mathcal{T}}$ given in (4.8), we get

$$
\begin{aligned}
m_{\mathcal{D}} \operatorname{div}^{\mathcal{D}} \mathbf{v}^{\mathcal{T}} & =\frac{1}{2}\left[m_{\sigma}\left(\mathbf{v}_{\mathcal{L}} \cdot \mathbf{e}_{\boldsymbol{x}}-\mathbf{v}_{\mathcal{K}} \cdot \mathbf{e}_{\boldsymbol{x}}\right)+m_{\sigma^{*}}\left(\mathbf{v}_{\mathcal{L}^{*}} \cdot \mathbf{e}_{\boldsymbol{y}}-\mathbf{v}_{\mathcal{K}^{*}} \cdot \mathbf{e}_{\boldsymbol{y}}\right)\right] \\
& =\frac{1}{2}\left[\int_{\gamma_{\mathcal{L}}^{v}} \mathbf{v}^{v} \cdot \mathbf{e}_{\boldsymbol{x}}-\int_{\gamma_{\mathcal{K}}^{v}} \mathbf{v}^{v} \cdot \mathbf{e}_{\boldsymbol{x}}+\int_{\gamma_{\mathcal{L}^{*}}^{v}} \mathbf{v}^{v} \cdot \mathbf{e}_{\boldsymbol{y}}-\int_{\gamma_{\mathcal{K}^{*}}^{v}} \mathbf{v}^{v} \cdot \mathbf{e}_{\boldsymbol{y}}\right] .
\end{aligned}
$$

We use the Stokes formula on the rectangle $R_{\mathcal{D}}$ whose sides are $\gamma_{\mathcal{K}}^{v}$, $\gamma_{\mathcal{K}^{*}}^{v}, \gamma_{\mathcal{L}}^{v}$ and $\gamma_{\mathcal{L}^{*}}^{v}$ and we get

$$
m_{\mathcal{D}} \operatorname{div}^{\mathcal{D}}\left(\mathbf{v}^{\mathcal{T}}\right)=\frac{1}{2} \int_{R_{\mathcal{D}}} \operatorname{div} \mathbf{v}^{v}
$$

Since $\mathcal{D}$ is a vertical diamond cell, we observe that $R_{\mathcal{D}} \backslash \mathcal{D}$ is included in the union of all the horizontal diamond cells. By assumption (4.3), we deduce that $\operatorname{div} \mathbf{v}^{v}$ is zero on $R_{\mathcal{D}} \backslash \mathcal{D}$. Moreover, using again (4.3) we get $\operatorname{div} \mathbf{v}^{h}=0$ on $\mathcal{D}$. It follows that

$$
m_{\mathcal{D}} \operatorname{div}^{\mathcal{D}}\left(\mathbf{v}^{\mathcal{T}}\right)=\int_{\mathcal{D}} \operatorname{div}\left(\frac{\mathbf{v}^{v}+\mathbf{v}^{h}}{2}\right)=\int_{\mathcal{D}} \operatorname{div} \mathbf{v}^{v, h} .
$$

Therefore, the corresponding term in the left-hand side of (4.5) is simply zero.

- The case where $\mathcal{D}$ is a boundary diamond cell. We assume for instance that $\mathcal{D} \in \mathfrak{D}^{v} \cap \mathfrak{D}_{\text {ext }}$, since the case of a boundary horizontal diamond cell is similar. We refer to Figure 12 for the notations.

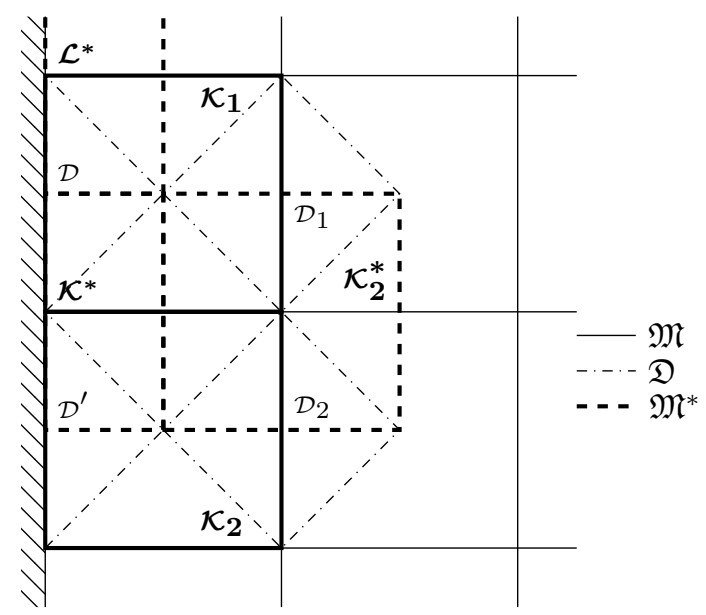

FiguRE 12. Notations for the study of the contribution of boundary diamond cells 
Since $\mathbf{v}^{\mathcal{T}} \in \mathbb{E}_{0}$, we have $\mathbf{v}_{\mathcal{K}^{*}}=\mathbf{v}_{\mathcal{L}^{*}}=0$ and, moreover, since $\mathbf{v}^{v}$ is supposed to be zero on $\partial \Omega$ and $\gamma_{\mathcal{L}}^{v} \subset \partial \Omega$, we have

$$
\mathbf{v}_{\mathcal{K}_{1}} \cdot \mathbf{e}_{\boldsymbol{x}}=\frac{1}{m_{\gamma_{\mathcal{K}_{1}}^{v}}} \int_{\gamma_{\mathcal{K}_{1}}^{v}} \mathbf{v}^{v} \cdot \mathbf{e}_{\boldsymbol{x}} \text { and } \mathbf{v}_{\mathcal{L}} \cdot \mathbf{e}_{\boldsymbol{x}}=0=\frac{1}{m_{\gamma_{\mathcal{L}}^{v}}} \int_{\gamma_{\mathcal{L}}^{v}} \mathbf{v}^{v} \cdot \mathbf{e}_{\boldsymbol{x}}
$$

It follows that

$$
\begin{aligned}
m_{\mathcal{D}} \operatorname{div}^{\mathcal{D}} \mathbf{v}^{\mathcal{T}} & =\frac{1}{2}\left[-\int_{\gamma_{\mathcal{L}}^{v}} \mathbf{v}^{v} \cdot \mathbf{e}_{\boldsymbol{x}}+\int_{\gamma_{\mathcal{K}_{1}}^{v}} \mathbf{v}^{v} \cdot \mathbf{e}_{\boldsymbol{x}}\right] \\
& =\frac{1}{2} \int_{R_{\mathcal{D}}} \operatorname{div} \mathbf{v}^{v}+\frac{1}{2}\left(\int_{\gamma_{\mathcal{K}^{*}}^{v}} \mathbf{v}^{v} \cdot \mathbf{e}_{\boldsymbol{y}}-\int_{\gamma_{\mathcal{L}^{*}}^{v}} \mathbf{v}^{v} \cdot \mathbf{e}_{\boldsymbol{y}}\right),
\end{aligned}
$$

where $R_{\mathcal{D}}$ is the rectangle whose sides are $\gamma_{\mathcal{K}_{1}}^{v}, \gamma_{\mathcal{K}^{*}}^{v}, \gamma_{\mathcal{L}}^{v}$ and $\gamma_{\mathcal{L}^{*}}^{v}$. By the same argument as in the previous case, using (4.3), we get

$$
\int_{\mathcal{D}} p^{\mathcal{D}}\left(\operatorname{div}^{\mathcal{D}} \mathbf{v}^{\mathcal{T}}-\operatorname{div} \mathbf{v}^{v, h}\right)=\frac{1}{2} p^{\mathcal{D}}\left(\int_{\gamma_{\mathcal{K}^{*}}^{v}} \mathbf{v}^{v} \cdot \mathbf{e}_{\boldsymbol{y}}-\int_{\gamma_{\mathcal{L}^{*}}^{v}} \mathbf{v}^{v} \cdot \mathbf{e}_{\boldsymbol{y}}\right) .
$$

Summing all the contributions of the boundary vertical diamond cells, we get

$$
\begin{aligned}
& \sum_{\mathcal{D} \in \mathfrak{D}} \int_{\mathcal{D} \in \mathfrak{D}^{v} \cap \mathfrak{D}_{\mathrm{ext}}} p^{\mathcal{D}}\left(\operatorname{div}^{\mathcal{D}} \mathbf{v}^{\mathcal{T}}-\operatorname{div} \mathbf{v}^{v, h}\right) \\
& =\frac{1}{2} \sum_{\mathcal{D} \in \mathcal{D}^{v} \cap \mathcal{D}_{\mathrm{ext}}} p^{\mathcal{D}}\left(\int_{\gamma_{\mathcal{K}^{*}}^{v}} \mathbf{v}^{v} \cdot \mathbf{e}_{\boldsymbol{y}}-\int_{\gamma_{\mathcal{L}^{*}}^{v}} \mathbf{v}^{v} \cdot \mathbf{e}_{\boldsymbol{y}}\right) \\
& =\frac{1}{2} \sum_{\substack{\mathcal{K}^{*} \in \partial \mathfrak{M}^{*} \\
x_{\mathcal{K}^{*} \in \partial \Omega^{v}}}}\left(p^{\mathcal{D}}-p^{\mathcal{D}^{\prime}}\right)\left(\int_{\gamma_{\mathcal{K}^{*}}^{v}} \mathbf{v}^{v} \cdot \mathbf{e}_{\boldsymbol{y}}\right),
\end{aligned}
$$

where $\mathcal{D}$ and $\mathcal{D}^{\prime}$ are the two diamond cells touching $x_{\mathcal{K}^{*}}$ as shown on Figure 12 .

Using the notations of Figure 12, for any $\mathcal{K}^{*} \in \partial \mathfrak{M}^{*}$ such that $x_{\mathcal{K}^{*}} \in$ $\partial \Omega^{v}$ we see that the chain $p^{\mathcal{D}} \stackrel{\mathcal{K}_{1}}{\longrightarrow} p^{\mathcal{D}_{1}} \stackrel{\mathcal{K}_{2}^{*}}{\longrightarrow} p^{\mathcal{D}_{2}} \stackrel{\mathcal{K}_{2}}{\longrightarrow} p^{\mathcal{D}^{\prime}}$ holds. It follows that

$$
\left|p^{\mathcal{D}}-p^{\mathcal{D}^{\prime}}\right| \leq C(\operatorname{reg}(\mathcal{T})) \operatorname{size}(\mathcal{T})\left(\left|\nabla^{\mathcal{K}_{1}} p^{\mathfrak{D}}\right|+\left|\nabla^{\mathcal{K}_{2}} p^{\mathfrak{D}}\right|+\left|\nabla^{\mathcal{K}_{2}^{*}} p^{\mathfrak{D}}\right|\right) .
$$

Moreover, for any $\mathcal{D} \in \mathfrak{D}^{v} \cap \mathfrak{D}_{\text {ext }}$, and according to (4.7) in Lemma 4.6. we have

$$
|\frac{1}{m_{\gamma_{\mathcal{K}^{*}}^{v}}} \int_{\gamma_{\mathcal{K}^{*}}^{v}} \mathbf{v}^{v} \cdot \mathbf{e}_{\boldsymbol{y}}-\underbrace{\frac{1}{m_{\gamma_{\mathcal{L}}^{v}}} \int_{\gamma_{\mathcal{L}}^{v}} \mathbf{v}^{v} \cdot \mathbf{e}_{\boldsymbol{y}}}_{=0}| \leq \frac{C(\operatorname{reg}(\mathcal{T}))}{m_{\gamma_{\mathcal{K}^{*}}^{v}}} \int_{\mathcal{K}_{1} \cup \mathcal{K}_{2}}\left|\nabla \mathbf{v}^{v}\right|
$$

so that finally, by using the Cauchy-Schwarz inequality

$$
\left|\sum_{\substack{\mathcal{K}^{*} \in \partial \mathfrak{M}^{*} \\ x_{\mathcal{K}^{*} \in \partial \Omega^{v}}}}\left(p^{\mathcal{D}}-p^{\mathcal{D}^{\prime}}\right) \int_{\gamma_{\mathcal{K}^{*}}^{v}} \mathbf{v}^{v} \cdot \mathbf{e}_{\boldsymbol{y}}\right| \leq C\left\|\mathbf{v}^{v}\right\|_{H^{1}}\left\|h^{\mathcal{T}} \nabla^{\mathcal{T}} p^{\mathfrak{D}}\right\|_{\mathcal{T}, 2} .
$$


- In conclusion, we proved that

$$
\left|\sum_{\mathcal{D} \in \mathfrak{D}} \int_{\mathcal{D}} p^{\mathcal{D}}\left(\operatorname{div}^{\mathcal{D}} \mathbf{v}^{\mathcal{T}}-\operatorname{div} \mathbf{v}^{v, h}\right)\right| \leq C\left(\left\|\mathbf{v}^{h}\right\|_{H^{1}}+\left\|\mathbf{v}^{v}\right\|_{H^{1}}\right)\left\|h^{\mathcal{T}} \nabla^{\mathcal{T}} p^{\mathfrak{D}}\right\|_{\mathcal{T}, 2} .
$$

- Let us now prove (4.5) in the case of the non-conforming Cartesian mesh defined in Figure 7b. We begin by writing the term under study as follows:

$$
\begin{aligned}
& \sum_{\mathcal{D} \in \mathfrak{D}} \int_{\mathcal{D}} p^{\mathcal{D}}\left(\operatorname{div}^{\mathcal{D}} \mathbf{v}^{\mathcal{T}}-\operatorname{div} \mathbf{v}^{v, h}\right)=\frac{1}{2} \sum_{\substack{\mathcal{K}^{*} \in \partial \mathfrak{M}^{*} \\
x_{\mathcal{K}^{*} \in \partial \Omega^{v}}}}\left(p^{\mathcal{D}}-p^{\mathcal{D}^{\prime}}\right)\left(\int_{\gamma_{\mathcal{K}^{*}}^{v}} \mathbf{v}^{v} \cdot \mathbf{e}_{\boldsymbol{y}}\right) \\
& +\frac{1}{2} \sum_{\substack{\mathcal{K}^{*} \in \partial \mathfrak{M}^{*} \\
x_{\mathcal{K}^{*}} \in \partial \Omega^{h} \\
x_{\mathcal{K}^{*} \notin I}}}\left(p^{\mathcal{D}}-p^{\mathcal{D}^{\prime}}\right)\left(\int_{\gamma_{\mathcal{K}^{*}}^{h}} \mathbf{v}^{h} \cdot \mathbf{e}_{\boldsymbol{x}}\right)+T_{I} .
\end{aligned}
$$

The first two terms contain the contributions of the boundary diamond cells away from the interface, this is the same computation as in the conforming case. Those terms can be estimated the same as before.

It remains to compute and estimate the contributions of the diamond cells near the interface that we gathered in the term $T_{I}$.

We denote by $\mathfrak{D}^{I}$ the set of the diamond cells having at least one vertex on the interface. This set contains both horizontal and vertical diamond cells and we need to distinguish the two cases. A particular numbering of those diamond cells is given in Figure 13. In the same figure, we define vertical segments denoted by $\omega_{\bullet}^{ \pm}$and $\sigma_{\bullet}^{ \pm}$that will be useful in the proof.

Associated with each of these segments we introduce the following fluxes:

$$
\begin{aligned}
F_{i+1 / 2}^{+} & =\int_{\sigma_{i+1 / 2}^{+}} \mathbf{v}^{h} \cdot \mathbf{e}_{\boldsymbol{x}}, & F_{i}^{+} & =\int_{\sigma_{i}^{+}} \mathbf{v}^{h} \cdot \mathbf{e}_{\boldsymbol{x}}, \\
F_{i-1 / 2}^{-} & =\int_{\sigma_{i-1 / 2}^{-}} \mathbf{v}^{h} \cdot \mathbf{e}_{\boldsymbol{x}}, & F_{i}^{-} & =\int_{\sigma_{i}^{-}} \mathbf{v}^{h} \cdot \mathbf{e}_{\boldsymbol{x}}, \\
G_{i}^{+} & =\int_{\omega_{i}^{+}} \mathbf{v}^{v} \cdot \mathbf{e}_{\boldsymbol{x}}, & G_{i}^{-} & =\int_{\omega_{i}^{-}} \mathbf{v}^{v} \cdot \mathbf{e}_{\boldsymbol{x}} .
\end{aligned}
$$

We conventionally set, for simplicity,

$$
F_{N+1 / 2}^{-}=F_{N+1 / 2}^{+}, \quad F_{1 / 2}^{+}=F_{1 / 2}^{-}, \text {and } F_{N+1}^{-}=F_{N}^{+} .
$$

- We begin with the contribution of the diamonds in $\mathfrak{D}^{v} \cap \mathfrak{D}^{I}$. For instance, we consider the case where $\mathcal{D}=\mathcal{D}_{i}^{+}$for some $i \in\{1, \ldots, N-1\}$, according to Figure 14a and to the definition of the discrete divergence, we have

$$
\begin{aligned}
& m_{\mathcal{D}_{i}^{+}} \operatorname{div}^{\mathcal{D}_{i}^{+}} \mathbf{v}^{\mathcal{T}}=\frac{1}{2}\left(m_{\gamma_{\mathcal{K}_{i}^{+}}^{v}} \mathbf{v}_{\mathcal{K}_{i}^{+}} \cdot \mathbf{e}_{\boldsymbol{x}}-m_{\gamma_{\mathcal{K}_{i}^{+}}^{v}} \mathbf{v}_{\mathcal{K}_{i}^{l}} \cdot \mathbf{e}_{\boldsymbol{x}}\right.
\end{aligned}
$$

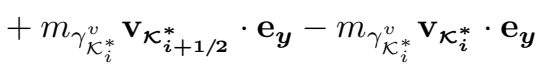

$$
\begin{aligned}
& \left.-\frac{1}{2} m_{\gamma_{\mathcal{K}_{i}^{+}}^{v}} \mathbf{v}_{\mathcal{K}_{i+1 / 2}^{*}} \cdot \mathbf{e}_{\boldsymbol{x}}+\frac{1}{2} m_{\gamma_{\mathcal{K}_{i}^{+}}^{v}} \mathbf{v}_{\mathcal{K}_{i}^{*}} \cdot \mathbf{e}_{\boldsymbol{x}}\right) .
\end{aligned}
$$




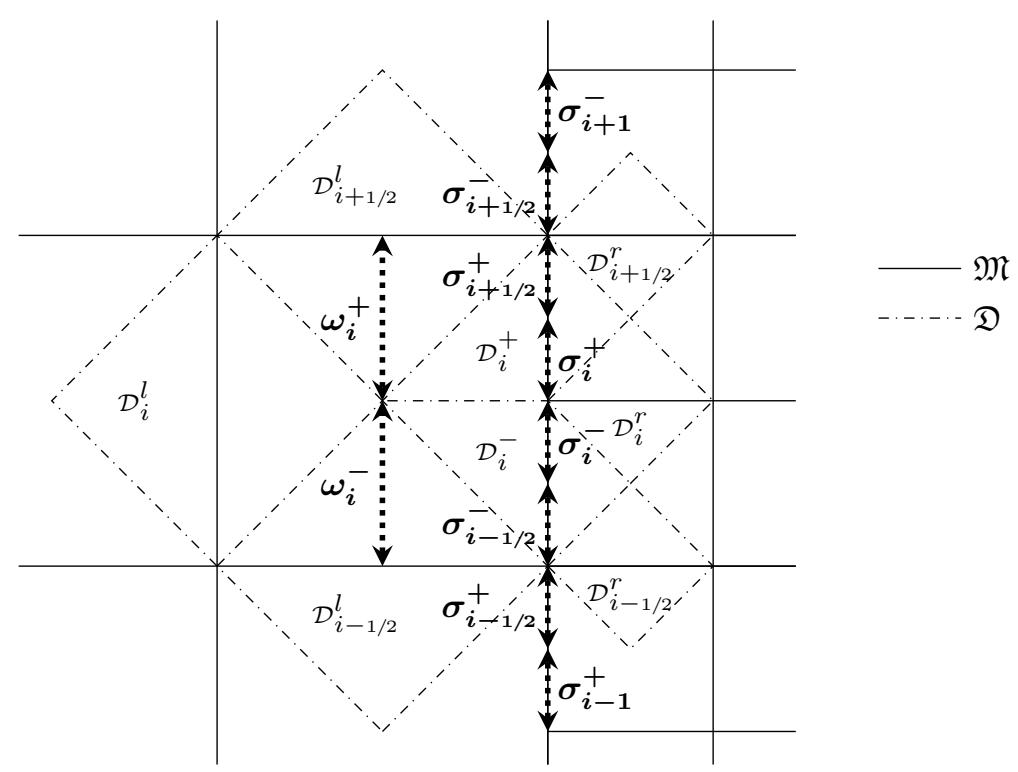

Figure 13. Definition of $\sigma_{\bullet}^{ \pm}$and $\omega_{\bullet}^{ \pm}$for the non-conforming Cartesian mesh

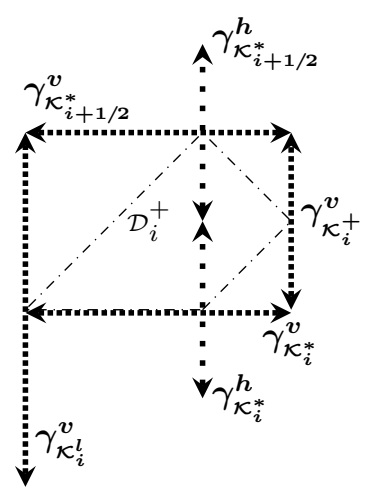

(a) The case of $\mathcal{D}_{i}^{+}$

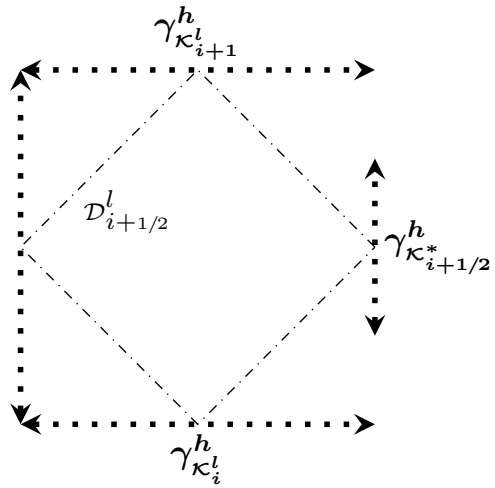

(b) The case of $\mathcal{D}_{i+1 / 2}^{l}$

Figure 14. Computation of the vertical interface diamond cells

By definition of $\mathbf{v}^{\mathcal{T}}$ given in (4.8), we obtain

$$
\begin{aligned}
m_{\mathcal{D}_{i}^{+}} \operatorname{div}^{\mathcal{D}_{i}^{+}} \mathbf{v}^{\mathcal{T}}=\frac{1}{2} & \left(\int_{\gamma_{\mathcal{K}_{i}^{+}}^{v}} \mathbf{v}^{v} \cdot \mathbf{e}_{\boldsymbol{x}}-\frac{1}{2} \int_{\gamma_{\mathcal{K}_{i}^{v}}^{v}} \mathbf{v}^{v} \cdot \mathbf{e}_{\boldsymbol{x}}\right. \\
& +\int_{\gamma_{\mathcal{K}_{i+1 / 2}^{*}}^{v}} \mathbf{v}^{v} \cdot \mathbf{e}_{\boldsymbol{y}}-\int_{\gamma_{\mathcal{K}_{i}^{*}}^{v}} \mathbf{v}^{v} \cdot \mathbf{e}_{\boldsymbol{y}} \\
& \left.+\frac{1}{2} \int_{\gamma_{\mathcal{K}_{i}^{*}}^{h}} \mathbf{v}^{h} \cdot \mathbf{e}_{\boldsymbol{x}}-\frac{1}{2} \int_{\gamma_{\mathcal{K}_{i+1 / 2}^{*}}^{h}} \mathbf{v}^{h} \cdot \mathbf{e}_{\boldsymbol{x}}\right) .
\end{aligned}
$$


If we denote by $R_{i}^{+}$the rectangle whose sides are $\gamma_{\mathcal{K}_{i}^{*}}^{v}, \gamma_{\mathcal{K}_{i}^{+}}^{v}, \gamma_{\mathcal{K}_{i+1 / 2}^{*}}^{v}$, $\omega_{i}^{+}$, and using (4.3), we get

$$
\begin{aligned}
\int_{\mathcal{D}_{i}^{+}} \operatorname{div} \mathbf{v}^{v} & =\int_{R_{i}^{+}} \operatorname{div} \mathbf{v}^{v} \\
& =\int_{\gamma_{\mathcal{K}_{i}^{v}}^{v}} \mathbf{v}^{v} \cdot \mathbf{e}_{\boldsymbol{x}}-\int_{\omega_{i}^{+}} \mathbf{v}^{v} \cdot \mathbf{e}_{\boldsymbol{x}}+\int_{\gamma_{\mathcal{K}_{i+1 / 2}^{v}}} \mathbf{v}^{v} \cdot \mathbf{e}_{\boldsymbol{y}}-\int_{\gamma_{\mathcal{K}_{i}^{*}}^{v}} \mathbf{v}^{v} \cdot \mathbf{e}_{\boldsymbol{y}}
\end{aligned}
$$

By subtraction, and using that $\operatorname{div} \mathbf{v}^{h}=0$ in $\mathcal{D}_{i}^{+}$which is a vertical diamond cell and the definition of the fluxes (4.10), it follows that

$$
\int_{\mathcal{D}_{i}^{+}}\left(\operatorname{div}^{\mathcal{D}_{i}^{+}} \mathbf{v}^{\mathcal{T}}-\operatorname{div} \mathbf{v}^{v, h}\right)=\frac{1}{4}\left(G_{i}^{+}-G_{i}^{-}+F_{i}^{+}+F_{i}^{-}-F_{i+1 / 2}^{+}-F_{i+1 / 2}^{-}\right) .
$$

In the case where $\mathcal{D}=\mathcal{D}_{i}^{-}$for $i \in\{2, \ldots, N\}$, we obtain by similar computations that

$$
\int_{\mathcal{D}_{i}^{-}}\left(\operatorname{div}^{\mathcal{D}_{i}^{-}} \mathbf{v}^{\mathcal{T}}-\operatorname{div} \mathbf{v}^{v, h}\right)=\frac{1}{4}\left(-G_{i}^{+}+G_{i}^{-}+F_{i}^{+}+F_{i}^{-}-F_{i-1 / 2}^{+}-F_{i-1 / 2}^{-}\right) .
$$

Finally, for the two boundary diamond cells $\mathcal{D}_{1}^{-}$and $\mathcal{D}_{N}^{+}$we get

$$
\begin{aligned}
& \int_{\mathcal{D}_{1}^{-}}\left(\operatorname{div}^{\mathcal{D}_{1}^{-}} \mathbf{v}^{\mathcal{T}}-\operatorname{div} \mathbf{v}^{v, h}\right)=\frac{1}{4}\left(-G_{1}^{+}+G_{1}^{-}+F_{1}^{+}+F_{1}^{-}\right), \\
& \int_{\mathcal{D}_{N}^{+}}\left(\operatorname{div}^{\mathcal{D}_{N}^{+}} \mathbf{v}^{\mathcal{T}}-\operatorname{div} \mathbf{v}^{v, h}\right)=\frac{1}{4}\left(G_{N}^{+}-G_{N}^{-}+F_{N}^{+}+F_{N}^{-}\right) .
\end{aligned}
$$

- Let us consider now the horizontal diamond cells touching the interface. We can easily see that, by definition of the segments $\gamma_{\mathcal{K}^{*}}^{h}$, such interior diamond cells located on the right of the interface (namely $\mathcal{D}_{i}^{r}$, $i=1, \ldots, N$ and $\mathcal{D}_{i+1 / 2}^{r}, i=1, \ldots, N-1$, see Figure 13) do not contribute to the sum under study. Actually, the computation is exactly the same as in the case of a uniform Cartesian mesh.

It remains to study the contributions of the interface horizontal diamond cells located on the left of the interface and refered to as $\mathcal{D}_{i+1 / 2}^{l}$ for $i=1, \ldots, N-1$; see Figure 14b.

Similar computations as the ones above give

$$
\int_{\mathcal{D}_{i+1 / 2}^{l}}\left(\operatorname{div}^{\mathcal{D}_{i+1 / 2}^{l}} \mathbf{v}^{\mathcal{T}}-\operatorname{div} \mathbf{v}^{v, h}\right)=\frac{1}{2}\left(F_{i+1 / 2}^{+}+F_{i+1 / 2}^{-}-F_{i}^{+}-F_{i+1}^{-}\right) .
$$


Gathering all these terms, we are led to the following expression of the interface term:

$$
\begin{aligned}
T_{I}= & \frac{1}{4} \sum_{i=1}^{N} p^{\mathcal{D}_{i}^{+}}\left(G_{i}^{+}-G_{i}^{-}+F_{i}^{+}+F_{i}^{-}-F_{i+1 / 2}^{+}-F_{i+1 / 2}^{-}\right) \\
& +\frac{1}{4} \sum_{i=1}^{N} p^{\mathcal{D}_{i}^{-}}\left(-G_{i}^{+}+G_{i}^{-}+F_{i}^{+}+F_{i}^{-}-F_{i-1 / 2}^{+}-F_{i-1 / 2}^{-}\right) \\
& +\frac{1}{2} \sum_{i=1}^{N-1} p^{\mathcal{D}_{i+1 / 2}^{l}}\left(F_{i+1 / 2}^{+}+F_{i+1 / 2}^{-}-F_{i}^{+}-F_{i+1}^{-}\right) \\
& -\frac{1}{2} p^{\mathcal{D}_{1 / 2}^{l}}\left(F_{1 / 2}^{-}+F_{1}^{-}\right)+\frac{1}{2} p^{\mathcal{D}_{1}^{-}} F_{1 / 2}^{-}+\frac{1}{2} p^{\mathcal{D}_{1 / 2}^{r}} F_{1 / 2}^{-} \\
& -\frac{1}{2} p^{\mathcal{D}_{N+1 / 2}^{l}}\left(F_{N+1 / 2}^{+}+F_{N}^{+}\right)+\frac{1}{2} p^{\mathcal{D}_{N}^{+}} F_{N+1 / 2}^{+}+\frac{1}{2} p^{\mathcal{D}_{N+1 / 2}^{r}} F_{N+1 / 2}^{+} .
\end{aligned}
$$

In this computation, we have taken care of the fact that a part of the contribution of the boundary diamonds $\mathcal{D}_{1 / 2}^{l}, \mathcal{D}_{1 / 2}^{r}, \mathcal{D}_{N+1 / 2}^{l}$ and $\mathcal{D}_{N+1 / 2}^{r}$ have already been taken into account in the second term of the right-hand side of (4.9).

We can now reorganize all these terms in the following way:

$$
\begin{aligned}
4 T_{I}= & \sum_{i=1}^{N}\left(p^{\mathcal{D}_{i}^{+}}-p^{\mathcal{D}_{i}^{-}}\right)\left(G_{i}^{+}-G_{i}^{-}\right) \\
& +\sum_{i=1}^{N-1}\left(2 p^{\mathcal{D}_{i+1 / 2}^{l}}-p^{\mathcal{D}_{i}^{+}}-p^{\mathcal{D}_{i+1}^{-}}\right)\left(F_{i+1 / 2}^{+}+F_{i+1 / 2}^{-}-F_{i}^{+}-F_{i+1}^{-}\right) \\
& +\sum_{i=1}^{N-1}\left(F_{i+1}^{-}-F_{i}^{+}\right)\left(p^{\mathcal{D}_{i+1}^{-}}-p^{\mathcal{D}_{i}^{-}}\right) \\
& +\sum_{i=1}^{N}\left(F_{i}^{-}-F_{i+1}^{-}\right)\left(p^{\mathcal{D}_{i}^{+}}-p^{\mathcal{D}_{i}^{-}}\right) \\
& +2 F_{1}^{-}\left(p^{\mathcal{D}_{1}^{-}}-p^{\mathcal{D}_{1 / 2}^{l}}\right)+2 F_{1 / 2}^{-}\left(p^{\mathcal{D}_{1 / 2}^{r}}-p^{\mathcal{D}_{1 / 2}^{l}}\right) \\
& +2 F_{N}^{+}\left(p^{\mathcal{D}_{N}^{+}}-p^{\mathcal{D}_{N+1 / 2}^{l}}\right)+2 F_{N+1 / 2}^{+}\left(p^{\mathcal{D}_{N+1 / 2}^{r}}-p^{\mathcal{D}_{N+1 / 2}^{l}}\right) .
\end{aligned}
$$

In this formula, the difference of fluxes can be estimated, as before, in terms of velocity gradients thanks to Lemma 4.6. It thus remains to bound all the pressure differences involved in this formula by means of DDFV pressure gradients.

For $i \in\{1, \ldots, N\}$, according to Figure 9 we have

$$
\begin{aligned}
m_{\mathcal{K}_{i+1 / 2}^{*}} \nabla^{\mathcal{K}_{i+1 / 2}^{*}} p^{\mathfrak{D}}= & \left(-h p^{\mathcal{D}_{i+1 / 2}^{l}}+\frac{h}{2} p^{\mathcal{D}_{i+1 / 2}^{r}}+\frac{h}{4}\left(p^{\mathcal{D}_{i+1}^{-}}+p^{\mathcal{D}_{i}^{+}}\right)\right) \mathbf{e}_{\boldsymbol{x}} \\
& +\frac{3 h}{4}\left(p^{\mathcal{D}_{i+1}^{-}}-p^{\mathcal{D}_{i}^{+}}\right) \mathbf{e}_{\boldsymbol{y}}
\end{aligned}
$$


so that

$$
\begin{aligned}
2 p^{\mathcal{D}_{i+1 / 2}^{l}}-p^{\mathcal{D}_{i}^{+}}-p^{\mathcal{D}_{i+1}^{-}}= & \frac{1}{2}\left(p^{\mathcal{D}_{i+1 / 2}^{r}}-p^{\mathcal{D}_{i}^{+}}\right)+\frac{1}{2}\left(p^{\mathcal{D}_{i+1 / 2}^{r}}-p^{\mathcal{D}_{i+1}^{-}}\right) \\
& -\frac{2}{h} m_{\mathcal{K}_{i+1 / 2}^{*}} \nabla^{\mathcal{K}_{i+1 / 2}^{*}} p^{\mathfrak{D}} \cdot \mathbf{e}_{\boldsymbol{x}} .
\end{aligned}
$$

Moreover, the following chains hold:

$$
\begin{array}{lllll}
p^{\mathcal{D}_{i+1}^{-}} & \stackrel{\mathcal{K}_{i+1 / 2}^{*}}{\longrightarrow} & p^{\mathcal{D}_{i}^{+}} & \stackrel{\mathcal{K}_{i}^{*}}{\longrightarrow} & p^{\mathcal{D}_{i}^{-}}, \\
p^{\mathcal{D}_{i+1 / 2}^{r}} & \stackrel{\mathcal{K}_{i}^{+}}{\longrightarrow} & p^{\mathcal{D}_{i}^{r}} & \stackrel{\mathcal{K}_{i}^{*}}{\longrightarrow} & p^{\mathcal{D}_{i}^{+}}, \\
p^{\mathcal{D}_{i+1 / 2}^{r}} & \stackrel{\mathcal{K}_{i+1}^{-}}{\longrightarrow} & p^{\mathcal{D}_{i+1}^{r}} & \stackrel{\mathcal{K}_{i+1}^{*}}{\longrightarrow} & p^{\mathcal{D}_{i+1}^{-}} .
\end{array}
$$

We also have to bound the differences $p^{\mathcal{D}_{1}^{-}}-p^{\mathcal{D}_{1 / 2}^{l}}, p^{\mathcal{D}_{1 / 2}^{r}}-p^{\mathcal{D}_{1 / 2}^{l}}, p^{\mathcal{D}_{N}^{+}}-$ $p^{\mathcal{D}_{N+1 / 2}^{l}}$ and $p^{\mathcal{D}_{N+1 / 2}^{r}}-p^{\mathcal{D}_{N+1 / 2}^{l}}$, corresponding to boundary terms. Let us for instance detail the reasoning for $p^{\mathcal{D}_{1}^{-}}-p^{\mathcal{D}_{1 / 2}^{l}}$ and $p^{\mathcal{D}_{1 / 2}^{r}}-p^{\mathcal{D}_{1 / 2}^{l}}$. We simply write

$$
\begin{aligned}
p^{\mathcal{D}_{1 / 2}^{l}}-p^{\mathcal{D}_{1}^{-}}= & \left(p^{\mathcal{D}_{1 / 2}^{l}}-p^{\mathcal{D}_{3 / 2}^{l}}\right)+\frac{1}{2}\left(p^{\mathcal{D}_{3 / 2}^{l}}-p^{\mathcal{D}_{3 / 2}^{r}}\right)+\frac{1}{2}\left(p^{\mathcal{D}_{3 / 2}^{l}}-p^{\mathcal{D}_{1}^{+}}\right) \\
& +\frac{1}{2}\left(p^{\mathcal{D}_{1}^{+}}-p^{\mathcal{D}_{1}^{-}}\right)+\frac{1}{2}\left(p^{\mathcal{D}_{3 / 2}^{r}}-p^{\mathcal{D}_{1}^{r}}\right)+\frac{1}{2}\left(p^{\mathcal{D}_{1}^{r}}-p^{\mathcal{D}_{1}^{-}}\right)
\end{aligned}
$$

and

$$
\begin{aligned}
p^{\mathcal{D}_{1 / 2}^{l}}-p^{\mathcal{D}_{1 / 2}^{r}}= & \left(p^{\mathcal{D}_{1 / 2}^{l}}-p^{\mathcal{D}_{3 / 2}^{l}}\right)+\frac{1}{2}\left(p^{\mathcal{D}_{3 / 2}^{l}}-p^{\mathcal{D}_{3 / 2}^{r}}\right)+\frac{1}{2}\left(p^{\mathcal{D}_{3 / 2}^{l}}-p^{\mathcal{D}_{1}^{+}}\right) \\
& +\frac{1}{2}\left(p^{\mathcal{D}_{1}^{+}}-p^{\mathcal{D}_{1}^{r}}\right)+\frac{1}{2}\left(p^{\mathcal{D}_{3 / 2}^{r}}-p^{\mathcal{D}_{1}^{r}}\right)+\left(p^{\mathcal{D}_{1}^{r}}-p^{\mathcal{D}_{1 / 2}^{r}}\right) .
\end{aligned}
$$

According to (4.12), specified to the dual cell $\mathcal{K}_{3 / 2}^{*}$, we have

$$
\begin{aligned}
& \frac{1}{2}\left(p^{\mathcal{D}_{3 / 2}^{l}}-p^{\mathcal{D}_{3 / 2}^{r}}\right)+\frac{1}{2}\left(p^{\mathcal{D}_{3 / 2}^{l}}-p^{\mathcal{D}_{1}^{+}}\right) \\
& =\frac{1}{h}\left(\frac{1}{3} m_{\mathcal{K}_{3 / 2}^{*}} \nabla^{\mathcal{K}_{3 / 2}^{*}} p^{\mathfrak{D}} \cdot \mathbf{e}_{\boldsymbol{y}}-m_{\mathcal{K}_{3 / 2}^{*}} \nabla^{\mathcal{K}_{3 / 2}^{*}} p^{\mathfrak{D}} \cdot \mathbf{e}_{\boldsymbol{x}}\right) .
\end{aligned}
$$

Furthermore, the following relations hold:

$$
\begin{aligned}
& p^{\mathcal{D}_{1 / 2}^{l}} \stackrel{\mathcal{K}_{1}^{l}}{\longrightarrow} p^{\mathcal{D}_{3 / 2}^{l},} \\
& p^{\mathcal{D}_{1}^{+}} \stackrel{\mathcal{K}_{1}^{*}}{\longrightarrow} p^{\mathcal{D}_{1}^{r}}, \quad p^{\mathcal{D}_{1}^{r}} \stackrel{\mathcal{K}_{1}^{*}}{\longrightarrow} p^{\mathcal{D}_{1}^{-}}, \\
& p^{\mathcal{D}_{3 / 2}^{r}} \stackrel{\mathcal{K}_{1}^{+}}{\longrightarrow} p^{\mathcal{D}_{1}^{r}}, \quad p^{\mathcal{D}_{1}^{r}} \stackrel{\mathcal{K}_{1}^{-}}{\longrightarrow} p^{\mathcal{D}_{1 / 2}^{r}} .
\end{aligned}
$$

To conclude, we use all the above estimates of pressure differences and Lemma 4.6 in formula (4.11) as well as the Cauchy-Schwarz inequality to obtain

$$
\left|T_{I}\right| \leq C\left(\left\|\mathbf{v}^{h}\right\|_{H^{1}}+\left\|\mathbf{v}^{v}\right\|_{H^{1}}\right)\left\|h^{\mathcal{T}} \nabla^{\mathcal{T}} p^{\mathfrak{D}}\right\|_{\mathcal{T}, 2},
$$

and the theorem is proved by coming back to (4.9).

We are now able to prove that, asymptotically, the unstable mode $q^{\mathfrak{D}}$ is essentially equal to the checkerboard mode. We recall that, according to (2.6), the unstable mode $q^{\mathfrak{D}}$ is completely characterized by the formula

$$
\beta_{\mathcal{T}}=\sup _{\mathbf{v}^{\mathcal{T}} \in \mathbb{E}_{0}} \frac{b\left(\mathbf{v}^{\mathcal{T}}, q^{\mathfrak{D}}\right)}{\left\|\nabla^{\mathfrak{D}} \mathbf{v}^{\mathcal{T}}\right\|_{\mathfrak{D}, 2}}
$$


with $m\left(q^{\mathfrak{D}}\right)=0$ and $\left\|q^{\mathfrak{D}}\right\|_{\mathfrak{D}, 2}=1$ and, for instance, the orientation condition $\left(q^{\mathfrak{D}}, \psi^{\mathfrak{D}}\right)>0$. The following result implies, in particular, that $q^{\mathfrak{D}}$ converges weakly (but not strongly) to 0 in $L^{2}(\Omega)$.

Theorem 4.7 (Unstable mode asymptotics). Let be $\mathcal{T}$ a non-conforming Cartesian $D D F V$ mesh as in Figure $7 \mathrm{~b}$ and let $q^{\mathfrak{D}}$ be the unstable mode numerically observed in Figure 10b. There exists $C_{8}>0$ which does not depend on $\operatorname{size}(\mathcal{T})$ such that

$$
\left\|q^{\mathfrak{D}}-\psi^{\mathfrak{D}}\right\|_{\mathfrak{D}, 2} \leq C_{8} \operatorname{size}(\mathcal{T})^{\frac{1}{2}}
$$

Proof. We set

$$
p^{\mathfrak{D}}=q^{\mathfrak{D}}-\left(q^{\mathfrak{D}}, \psi^{\mathfrak{D}}\right) \psi^{\mathfrak{D}},
$$

so that we get $\left(p^{\mathfrak{D}}, \psi^{\mathfrak{D}}\right)=0$ and $m\left(p^{\mathfrak{D}}\right)=0$. We apply Theorem 4.4 to obtain, with (4.13) and (4.14), that

$$
C_{7}\left\|p^{\mathfrak{D}}\right\|_{\mathfrak{D}, 2} \leq \sup _{\mathbf{v}_{\mathcal{T}}^{\mathcal{T}} \in \mathbb{E}_{0}} \frac{b\left(\mathbf{v}^{\mathcal{T}}, p^{\mathfrak{D}}\right)}{\left\|\nabla^{\mathfrak{D}} \mathbf{v}^{\mathcal{T}}\right\|_{\mathfrak{D}, 2}} \leq \beta_{\mathcal{T}}+\left(q^{\mathfrak{D}}, \psi^{\mathfrak{D}}\right) \sup _{\mathbf{v}^{\mathcal{T}} \in \mathbb{E}_{0}} \frac{b\left(\mathbf{v}^{\mathcal{T}}, \psi^{\mathfrak{D}}\right)}{\left\|\nabla^{\mathfrak{D}} \mathbf{v}^{\mathcal{T}}\right\|_{\mathfrak{D}, 2}}
$$

By observing that $\left\|\psi^{\mathfrak{D}}\right\|_{\mathfrak{D}, 2}=\left\|q^{\mathfrak{D}}\right\|_{\mathfrak{D}, 2}=1$ and $\left(q^{\mathfrak{D}}, \psi^{\mathfrak{D}}\right) \leq 1$, Theorem 4.3 implies

$$
\left\|q^{\mathfrak{D}}-\left(q^{\mathfrak{D}}, \psi^{\mathfrak{D}}\right) \psi^{\mathfrak{D}}\right\|_{\mathfrak{D}, 2} \leq 2 \frac{C_{6}}{C_{7}} \operatorname{size}(\mathcal{T})^{\frac{1}{2}} .
$$

Furthermore, we have $\left(q^{\mathfrak{D}}, \psi^{\mathfrak{D}}\right)^{2}=\left(\left(q^{\mathfrak{D}}, \psi^{\mathfrak{D}}\right) \psi^{\mathfrak{D}}-q^{\mathfrak{D}}, q^{\mathfrak{D}}\right)+\left\|q^{\mathfrak{D}}\right\|_{\mathfrak{D}, 2}^{2}$, and since $\left(p^{\mathfrak{D}}, \psi^{\mathfrak{D}}\right)=0$ and (4.15), we get

$$
\left(q^{\mathfrak{D}}, \psi^{\mathfrak{D}}\right)^{2}=1-\left\|q^{\mathfrak{D}}-\left(q^{\mathfrak{D}}, \psi^{\mathfrak{D}}\right) \psi^{\mathfrak{D}}\right\|_{\mathfrak{D}, 2}^{2} \geq 1-4 \frac{C_{6}^{2}}{C_{7}^{2}} \operatorname{size}(\mathcal{T}) .
$$

We conclude by observing that

$$
\left\|q^{\mathfrak{D}}-\psi^{\mathfrak{D}}\right\|_{\mathfrak{D}, 2} \leq\left\|q^{\mathfrak{D}}-\left(q^{\mathfrak{D}}, \psi^{\mathfrak{D}}\right) \psi^{\mathfrak{D}}\right\|_{\mathfrak{D}, 2}+\left(1-\left(q^{\mathfrak{D}}, \psi^{\mathfrak{D}}\right)\right) .
$$

\section{Further NUmericAl RESUlts AND CONCLUSION}

(1) We first consider other kinds of non-conforming Cartesian meshes of the unit square $\Omega=] 0,1\left[^{2}\right.$ as shown in Figure [15. Note that these meshes present more than one non-conformity interface.

We observe the same results as in the previous case, namely that the Inf-Sup constant $\beta_{\mathcal{T}}$ tends to 0 and that $\sqrt{\lambda_{3}\left(S_{\mathcal{T}}\right)}$ is bounded from below when $\operatorname{size}(\mathcal{T})$ tends to 0 . In both cases, we deduce that the codimension 1 stability property also holds. We also observe that the unstable mode in each case has again the shape of a checkerboard mode. Actually, one can check that the theoretical results proved in Section 4 can be adapted to these geometries.

(2) We investigate now the Inf-Sup stability property for two families of conforming meshes corresponding to various subdomains with either a uniform Cartesian mesh, or a triangle mesh (see Figures 16a and 16b).

Our results (see Figure 17) show that $\beta_{\mathcal{T}}$ remains away from 0 when $\operatorname{size}(\mathcal{T})$ tends to 0 , and therefore that the DDFV scheme is Inf-Sup stable for such families of meshes. However, it seems that the adaptation of the previous proofs to those cases is not straightforward and thus will be the object of a forthcoming work. 


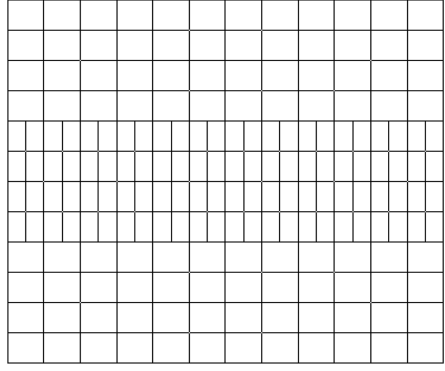

(a) Mesh with two interfaces

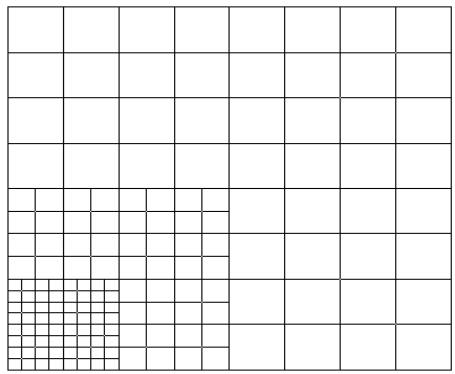

(c) Locally refined mesh

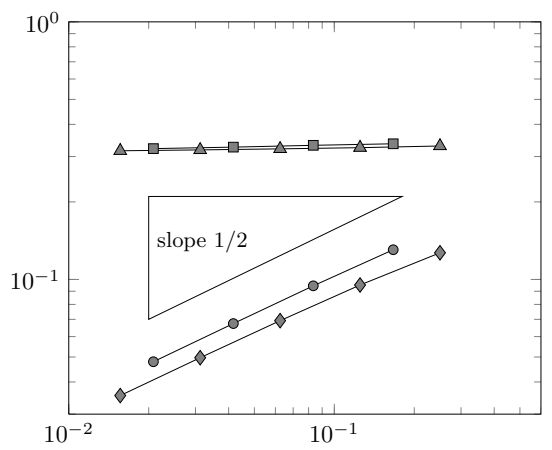

(e) The discrete Inf-Sup constant $\beta_{\mathcal{T}}=\sqrt{\lambda_{2}\left(S_{\mathcal{T}}\right)}$ and $\sqrt{\lambda_{3}\left(S_{\mathcal{T}}\right)}$ as a function of $\operatorname{size}(\mathcal{T})$

FiguRE 15. Other non-conforming Cartesian meshes

We also observe in Figure 17 that the DDFV method seems to be Inf-Sup stable for mixed triangle/quadrangle meshes (Figure 16c) but also for more general polygonal meshes constituted by hexagons for instance (Figure 16d). 


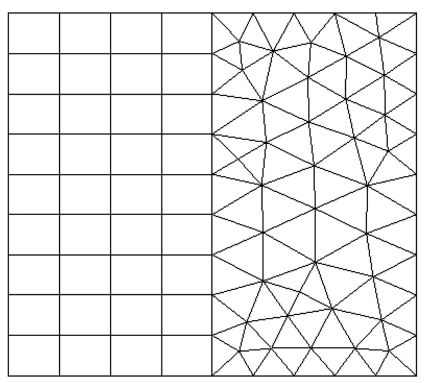

(a) Mesh with one interface

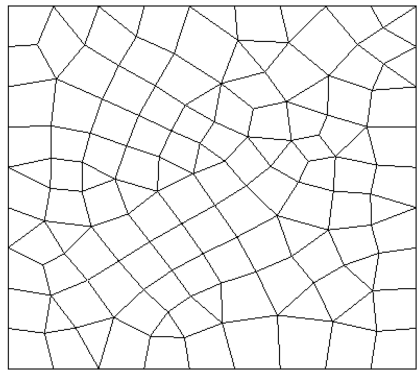

(c) Conforming quadrangle and triangle mesh

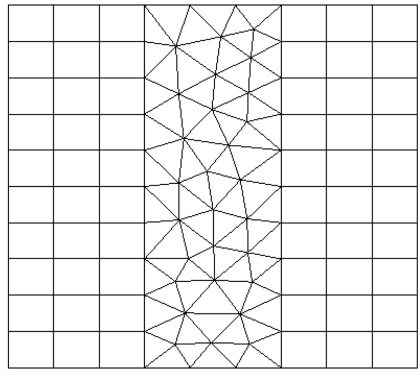

(b) Mesh with two interfaces

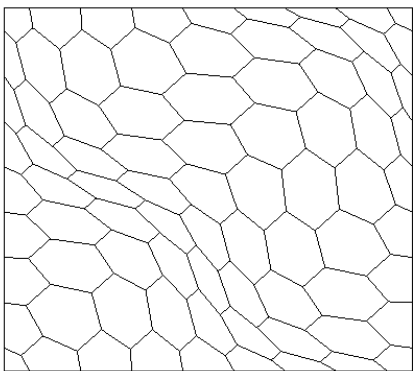

(d) Hexagon mesh

FIGURE 16. Various kinds of meshes

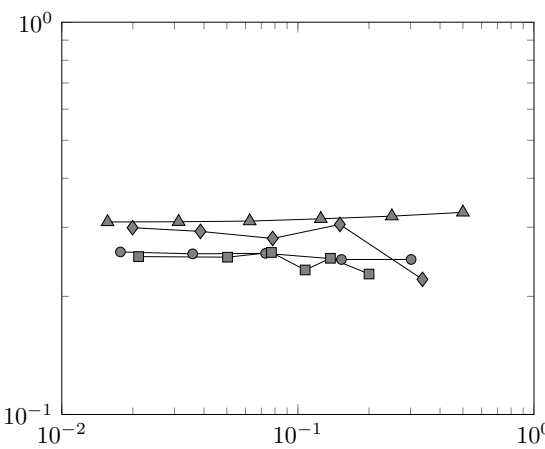

Mesh with one interface, Figure 16a- -

Mesh with two interfaces, Figure 16b- $\square-$ Conforming quadrangle and triangle mesh, Figure $16 \mathrm{c}-\sim$ Hexagon mesh, Figure $16 \mathrm{~d}-\triangle$

Figure 17. The discrete Inf-Sup constant $\beta_{\mathcal{T}}=\sqrt{\lambda_{2}\left(S_{\mathcal{T}}\right)}$ as a function of $\operatorname{size}(\mathcal{T})$ 


\section{Conclusion}

In this paper, we have investigated from a numerical and theoretical point of view whether or not the Inf-Sup stability condition for the DDFV scheme holds for various kinds of mesh families. We observe that the DDFV scheme seems to be very robust as far as this stability property is concerned, in particular, in the case of non-conforming meshes. We managed to prove this property for different mesh families but the proof strongly depends on the geometry of the meshes. Up to now, we are not able to prove the stability for very general polygonal meshes, even though we have given numerical evidence that it should be true.

\section{REFERENCES}

[1] B. Andreianov, M. Bendahmane, F. Hubert, and S. Krell, On 3D DDFV discretization of gradient and divergence operators. I. Meshing, operators and discrete duality, IMA J. Numer. Anal. 32 (2012), no. 4, 1574-1603, DOI 10.1093/imanum/drr046. MR2991838

[2] B. Andreianov, F. Boyer, and F. Hubert, Discrete duality finite volume schemes for LerayLions-type elliptic problems on general 2D meshes, Numer. Methods Partial Differential Equations 23 (2007), no. 1, 145-195, DOI 10.1002/num.20170. MR2275464 (2008c:65283)

[3] D. N. Arnold, F. Brezzi, and M. Fortin, A stable finite element for the Stokes equations, Calcolo 21 (1984), no. 4, 337-344 (1985), DOI 10.1007/BF02576171. MR799997|(86m:65136)

[4] L. Beirão da Veiga, V. Gyrya, K. Lipnikov, and G. Manzini, Mimetic finite difference method for the Stokes problem on polygonal meshes, J. Comput. Phys. 228 (2009), no. 19, 7215-7232, DOI 10.1016/j.jcp.2009.06.034. MR2568590(2010k:65229)

[5] L. Beirão da Veiga and K. Lipnikov, A mimetic discretization of the Stokes problem with selected edge bubbles, SIAM J. Sci. Comput. 32 (2010), no. 2, 875-893, DOI 10.1137/090767029. MR.2609344(2011e:65255)

[6] L. Beirão da Veiga, K. Lipnikov, and G. Manzini, Error analysis for a mimetic discretization of the steady Stokes problem on polyhedral meshes, SIAM J. Numer. Anal. 48 (2010), no. 4, 1419-1443, DOI 10.1137/090757411. MR2684341 (2011m:65246)

[7] D. Boffi, F. Brezzi, L. F. Demkowicz, R. G. Durán, R. S. Falk, and M. Fortin, Mixed Finite Elements, Compatibility Conditions, and Applications, Lecture Notes in Mathematics, vol. 1939, Springer-Verlag, Berlin; Fondazione C.I.M.E., Florence, 2008. Lectures given at the C.I.M.E. Summer School held in Cetraro, June 26-July 1, 2006; Edited by Boffi and Lucia Gastaldi. MR2459075 (2010h:65219)

[8] F. Boyer and P. Fabrie, Mathematical Tools for the Study of the Incompressible Navier-Stokes Equations and Related Models, Applied Mathematical Sciences, vol. 183, Springer, New York, 2013. MR2986590

[9] F. Brezzi and M. Fortin, Mixed and Hybrid Finite Element Methods, Springer Series in Computational Mathematics, vol. 15, Springer-Verlag, New York, 1991. MR1115205(92d:65187)

[10] B. Cockburn, G. Kanschat, D. Schötzau, and C. Schwab, Local discontinuous Galerkin methods for the Stokes system, SIAM J. Numer. Anal. 40 (2002), no. 1, 319-343 (electronic), DOI 10.1137/S0036142900380121. MR1921922 (2003g:65141)

[11] Y. Coudière and F. Hubert, A 3D discrete duality finite volume method for nonlinear elliptic equations, SIAM J. Sci. Comput. 33 (2011), no. 4, 1739-1764, DOI 10.1137/100786046. MR2831032(2012m:65378)

[12] Y. Coudière, C. Pierre, O. Rousseau, and R. Turpault, A 2D/3D discrete duality finite volume scheme. Application to ECG simulation, Int. J. Finite Vol. 6 (2009), no. 1, 24. MR2500950 (2010e:65187)

[13] M. Crouzeix and P.-A. Raviart, Conforming and nonconforming finite element methods for solving the stationary Stokes equations. I, Rev. Française Automat. Informat. Recherche Opérationnelle Sér. Rouge 7 (1973), no. R-3, 33-75. MR0343661 (49 \#8401)

[14] S. Delcourte, Développement de méthodes de volumes finis pour la mécanique des fluides, Ph.D. thesis, http://tel.archives-ouvertes.fr/tel-00200833/fr/, Université Paul Sabatier, Toulouse, France, 2007. 
[15] D. A. Di Pietro and A. Ern, Mathematical Aspects of Discontinuous Galerkin Methods, Mathématiques \& Applications (Berlin) [Mathematics \& Applications], vol. 69, Springer, Heidelberg, 2012. MR2882148

[16] K. Domelevo and P. Omnes, A finite volume method for the Laplace equation on almost arbitrary two-dimensional grids, M2AN Math. Model. Numer. Anal. 39 (2005), no. 6, 12031249, DOI 10.1051/m2an:2005047. MR2195910 (2006j:65312)

[17] J. Droniou and R. Eymard, Study of the mixed finite volume method for Stokes and NavierStokes equations, Numer. Methods Partial Differential Equations 25 (2009), no. 1, 137-171, DOI 10.1002/num.20333. MR2473683(2009k:65208)

[18] A. Ern and J.-L. Guermond, Theory and Practice of Finite Elements, Applied Mathematical Sciences, vol. 159, Springer-Verlag, New York, 2004. MR2050138 (2005d:65002)

[19] R. Eymard, T. Gallouët, and R. Herbin, Finite volume methods, Handbook of Numerical Analysis, Vol. VII, Handb. Numer. Anal., VII, North-Holland, Amsterdam, 2000, pp. 7131020. MR1804748(2002e:65138)

[20] R. Eymard, R. Herbin, and J. C. Latché, On a stabilized colocated finite volume scheme for the Stokes problem, M2AN Math. Model. Numer. Anal. 40 (2006), no. 3, 501-527, DOI 10.1051/m2an:2006024. MR2245319 (2007d:65100)

[21] M. Fortin, An analysis of the convergence of mixed finite element methods (English, with French summary), RAIRO Anal. Numér. 11 (1977), no. 4, 341-354, iii. MR0464543 (57 \#4473)

[22] V. Girault and P.-A. Raviart, Finite Element Methods for Navier-Stokes Equations, Springer Series in Computational Mathematics, vol. 5, Springer-Verlag, Berlin, 1986. Theory and algorithms. MR 851383 (88b:65129)

[23] V. Girault, B. Rivière, and M. F. Wheeler, A discontinuous Galerkin method with nonoverlapping domain decomposition for the Stokes and Navier-Stokes problems, Math. Comp. 74 (2005), no. 249, 53-84 (electronic), DOI 10.1090/S0025-5718-04-01652-7. MR2085402 (2005f:65149)

[24] F. Harlow and J. Welch, Numerical calculation of time-dependent viscous incompressible flow of fluid with free surface, The physics of fluids 8 (1965), no. 12, 2182-2189.

[25] F. Hermeline, Approximation of 2-D and 3-D diffusion operators with variable full tensor coefficients on arbitrary meshes, Comput. Methods Appl. Mech. Engrg. 196 (2007), no. 21-24, 2497-2526, DOI 10.1016/j.cma.2007.01.005. MR2319051(2008d:65123)

[26] S. Krell, Stabilized DDFV schemes for Stokes problem with variable viscosity on general 2D meshes, Numer. Methods Partial Differential Equations 27 (2011), no. 6, 1666-1706, DOI 10.1002/num.20603. MR2838314(2012j:65368)

[27] S. Krell and G. Manzini, The discrete duality finite volume method for Stokes equations on three-dimensional polyhedral meshes, SIAM J. Numer. Anal. 50 (2012), no. 2, 808-837, DOI 10.1137/110831593. MR2914287

[28] D. S. Malkus, Eigenproblems associated with the discrete LBB condition for incompressible finite elements, Internat. J. Engrg. Sci. 19 (1981), no. 10, 1299-1310, DOI 10.1016/00207225(81)90013-6. MR660563 (83k:73053)

[29] R. A. Nicolaides, Analysis and convergence of the MAC scheme. I. The linear problem, SIAM J. Numer. Anal. 29 (1992), no. 6, 1579-1591, DOI 10.1137/0729091. MR1191137(93j:65143)

[30] Y. Saad, Iterative Methods for Sparse Linear Systems, 2nd ed., Society for Industrial and Applied Mathematics, Philadelphia, PA, 2003. MR 1990645 (2004h:65002)

[31] R. Verfürth, Error estimates for a mixed finite element approximation of the Stokes equations, RAIRO Anal. Numér. 18 (1984), no. 2, 175-182. MR743884 (85i:65156)

Aix Marseille Université, CnRS, Centrale Marseille, I2M, UMR 7373, Marseille, FRANCE

E-mail address: franck.boyer@univ-amu.fr

Université de Nice Sophia-Antipolis, CNRS, LJAD UMR 7351, Nice, France

E-mail address: krell@unice.fr

Aix Marseille Université, CnRs, Centrale Marseille, I2M, UMR 7373, Marseille, FRANCE

E-mail address: flore.nabet@univ-amu.fr 